\title{
Computer Modeling of Halogen Bonds and Other $\sigma$-Hole Interactions
}

\author{
Michal H. Kolár $\check{r}^{*, \dagger} \ddagger$ and Pavel Hobza \\ $\dagger$ Institute of Organic Chemistry and Biochemistry, Academy of Sciences of the Czech \\ Republic, Flemingovo nám. 2, 16610 Prague, Czech Republic \\ ¥Institute of Neuroscience and Medicine (INM-9) and Institute for Advanced Simulations \\ (IAS-5), Forschungszentrum Jülich GmbH, 52428 Jülich, Federal Republic of Germany \\ \Department of Physical Chemistry, Regional Centre of Advanced Technologies and \\ Materials, Palacky University, 77146 Olomouc, Czech Republic \\ E-mail: michal.kolar@uochb.cas.cz \\ Phone: +420220410318
}

\section{Contents}
Abstract
3
1 Introduction
2 Nature of Halogen Bonding
$2.1 \quad \sigma$-Hole .............................. 7
2.1.1 Electrostatic Potential .................. 7
2.1.2 Discovery of $\sigma$-Hole . . . . . . . . . . . . . . . . . . . . . . . 9 9
2.1.3 $\sigma$-Holes on Halogens and Other Atoms . . . . . . . . . . . . . . 10
2.1.4 Characteristics of a $\sigma$-Hole . . . . . . . . . . . . . . 14 
2.1.5 Calculations of $\sigma$-Holes . . . . . . . . . . . . . . . . 19

2.1 .6 Interaction Energies and $\sigma$-Holes $\ldots \ldots \ldots \ldots \ldots$

2.2 Interaction Energy Decompositions $\ldots \ldots \ldots \ldots \ldots$

2.2.1 Symmetry-Adapted Perturbation Theory . . . . . . . . . . 26

2.2 .2 Other Schemes . . . . . . . . . . . . . . 35

3 Computational Methods 36

3.1 Benchmark Calculations and Performance of Various Computational Methods 36

$3.1 .1 \quad \mathrm{X} 40$ Data Set . . . . . . . . . . . . . . . . 39

3.1 .2 XB18 and XB51 Data Sets ................. 45

3.1 .3 Benchmarking Other $\sigma$-Hole Interactions . . . . . . . . . . 48

3.2 Semiempirical Methods . . . . . . . . . . . . . . . . . 50 50

3.3 Empirical Force Fields . . . . . . . . . . . . . . . . . . . . . . . . . . 52

3.3.1 Methods with Off-Center Point Charges . . . . . . . . . 54

3.3.2 Electric Multipole Expansion . . . . . . . . . . . . 56

3.3 .3 Aspherical Interatomic Potentials . . . . . . . . . . . . . 57

3.4 Methods for Virtual Screening . . . . . . . . . . . . . . . . 58 58

3.5 Semiempirical QM Scoring Functions . . . . . . . . . . . . . 60

4 Computational Studies 61

4.1 Directionality of $\sigma$-Hole Interactions . . . . . . . . . . . . . . 61

4.2 Hydrogen vs. Halogen Bonding . . . . . . . . . . . . . . . 65 65

4.3 Vibrational Spectra of Halogen-Bonded Complexes . . . . . . . . . . 67

4.4 Structural Studies of Small Molecules . . . . . . . . . . . . . . . 69

4.5 Halogen Bonds in Biomolecules and Medicinal Chemistry Applications ... 71

5 Concluding Remarks

6 Biographies $\quad \mathbf{7 6}$ 
6.1 Michal H. Kolár̆ . . . . . . . . . . . . . . . . . . . . . . . . 7 76

6.2 Pavel Hobza . . . . . . . . . . . . . . . . . . . . . . . . 776

$\begin{array}{ll}\text { Acronyms and Abbreviations } & 77\end{array}$

Acknowledgement

\begin{tabular}{lr} 
References & 80 \\
\hline
\end{tabular}

\begin{abstract}
In the field of noncovalent interactions a new paradigm has recently become popular. It stems from the analysis of molecular electrostatic potentials and introduces a label, which has recently attracted enormous attention. The label is $\sigma$-hole and it was first used in connection with halogens. It initiated a renascence of interest in halogenated compounds, and later on, when found also on other groups of atoms (chalcogens, pnicogens, tetrels and aerogens), resulted in a new direction of research of intermolecular interactions. In the review, we summarize advances from about last ten years in understanding those interactions related to $\sigma$-hole. We pay particular attention to theoretical and computational techniques, which play crucial role in the field.
\end{abstract}

\title{
1 Introduction
}

In 2013, the International Union of Pure and Applied Chemistry (IUPAC) presented a recommended definition of the halogen bond (XB). According to Desiraju et al., ${ }^{1}$ the halogen bond "occurs when there is evidence of a net attractive interaction between an electrophilic region associated with a halogen atom in a molecular entity and a nucleophilic region in another, or the same, molecular entity." A representative XB scheme with typical geometric features is shown in Figure 1. As discussed later, its stabilization energy typically reaches 


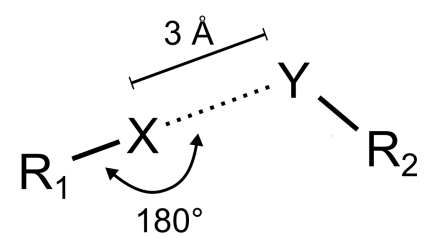

Figure 1: Schematic geometry of an idealized halogen bond between a halogen atom $\mathrm{X}$ (fluorine, chlorine, bromine, or iodine) and an electron-rich atom or functional group Y. Typical distance and intermolecular angle are depicted.

several kilocalories per mol.

Halogen bonding is an example of a broader class of noncovalent interactions that are characterized by the electrophilic region of one of the two interacting partners. These interactions have become known as $\sigma$-hole bonding.

The aim of this review is to summarize the last ten years of computational studies on XB and other noncovalent interactions involving $\sigma$-holes. Over this period, and perhaps even starting a few years beforehand, XB has become a true celebrity among noncovalent interactions. This is especially evident in the field of computational chemistry, which motivated us to sum up the most paramount achievements and critically comment on them. A number of reviews on XB from a variety of perspectives have been published. ${ }^{2} 14$ The reader might also be interested in a recent review of theoretical methods for describing XB. ${ }^{15} \sigma$-hole bonding has not been reviewed as extensively, $\frac{16}{19}$ and we attempt to contribute to this topic, while keeping our major focus on XB.

This review is organized as follows. After several introductory remarks, we continue with a subject of turbulent discussions - the nature of halogen bonding (Section 2). Here, we review the concept of $\sigma$-hole with special care to highlight this revolutionary way of explaining $\mathrm{XB}$ and explain its fundamental importance. Afterwards, we discuss a few relevant quantum chemical approaches. Section 3 is dedicated to a thorough description of the computational arsenal used to tackle XB, covering the range from highly accurate calculations to extremely fast and efficient empirical approaches. Finally, in Section 4 we describe several areas of XB research in which both computational techniques and experimental work have played roles. 

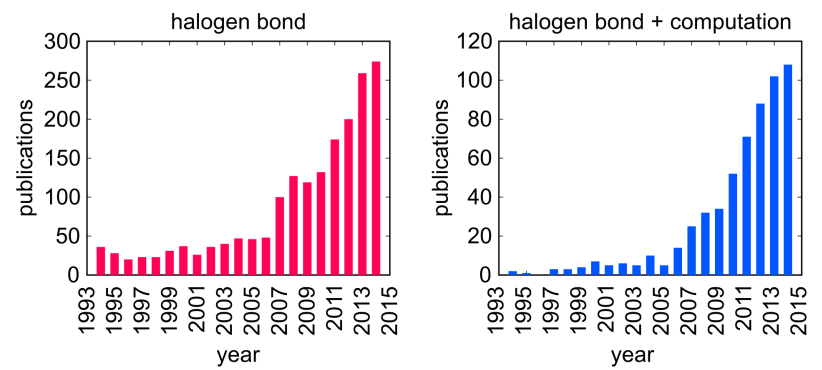

Figure 2: Number of publications about XB from the Thomson Reuters Web of Science (2015/04). Left: Publications including the keywords "halogen bond" or "halogen bonding." Right: The same with an additional keyword from a list evoking a computational or theoretical study (e.g., "density functional theory," "molecular modeling," etc.).

Several factors have contributed to the recent renaissance of XB. The first is the rapid growth of successful applications of XB in fields such as material design, 2021 drug development, $\frac{12[22[23}{\text { and catalysis. }}{ }^{24 \mid 25}$ These advances opened the door for additional funding and more detailed research of XB. Another, perhaps even more prominent, factor is the character of XB, which, although puzzling, can be explained in a simple manner to high school students. One must bear in mind that other noncovalent interactions have been included in their textbooks for decades, including the hydrophobic effect, $\stackrel{26 \mid 27}{2}$ the hydrogen bond a prototypic noncovalent interaction ${ }^{[28}$ - and van der Waals interactions. ${ }^{[290]}$ From the students' perspective is it is always more appealing to explore something which had not been known before.

The puzzling character of XB arose from the fact that because halogens are electronegative atoms, it was not easy to understand why they are attracted by electron-rich moieties such as carbonyl oxygens and $\pi$-electron systems. To this point, we think that the breakthrough in understanding XB that occurred about ten years ago was not purely scientific but stemmed more from a marketing perspective. As discussed in detail below, an elegant way of explaining many features of XB - the so-called $\sigma$-hole - appeared, or more precisely, got a name. A seminal paper by Clark et al. ${ }^{31}$ in 2007 triggered a new epoch of XB research (Figure 2).

Finally, from a computational point of view, another factor has played an important role 
- the ongoing improvement in both software and hardware ${ }^{\sqrt{32}}$ used to address questions in chemistry and other natural sciences. The level at which one needs to understand a theory to run computations has been decreasing. On one hand, in a positive sense, this allows people with limited programming and computational science abilities to focus on chemistry. On the other hand, it reduces the barrier for doing computational science, often negatively affecting the quality and/or impact of the output.

\section{Nature of Halogen Bonding}

The first thing we should address is why it is meaningful to investigate the nature of noncovalent interactions. What kinds of noncovalent interactions nature has? Is it valid to ask? The potential energy part of a molecular Hamiltonian contains only the Coulombic term, i.e., the reciprocal interparticle distance. Consequently, as stated by Feynman ${ }^{33}$ and argued by Politzer et al. in the context of $\sigma$-hole molecular interactions in several recent papers, $\frac{1734}{36}$ the only nature of any interatomic interaction is electrostatics, which encompasses many other commonly used contributions, including covalency, London dispersion forces, and polarization.

Their arguments are perfectly valid. For instance, from a physical perspective, there is no sharp border between covalent and noncovalent interactions, and thus there is no physical reason to separate them into different groups. On the other hand, it is natural for chemists (and not only for them) to distinguish between the interaction of two carbon atoms in ethane and the interaction of carbon atoms in two methane molecules. Therefore the chemist would say that there is a chemical bond in between the two carbon atoms, whereas there is nothing like chemical bonding in a strict physical view. Likewise, in the gas phase the interaction of two methanes is driven by other factors than in water solution. There would be hardly any doubts that the concept of chemical bond or hydrophobic effect is useful, however as Clark pointed out, "We sometimes lose track of the fact that many common concepts 
(e.g., hybridization, molecular orbitals, and resonance structures) are indeed simply models designed to rationalize what we observe." 118 It is natural to use schemes and simplifying patterns in our daily life observations as well as in chemistry. This way of complexity reduction is our main argument for why we think, and the experience of others seems to justify, that it is advantageous to adopt certain interaction energy decompositions to describe noncovalent complexes and classify them according to their nature.

In the course of time, a number of schemes have emerged to describe the physical reality of intermolecular binding (reviewed recently by Phipps et al. ${ }^{\text {[37) }}$. We can understand them as attempts to tell the same story using variety of words and diction perhaps targeting various listeners. Unfortunately, and this must be stressed, the decomposition of interaction energy is ambiguous; there seems to be no "more correct" energy decomposition alike there is no "more correct" way of telling a story. One can only choose the "more correct" approach to a listener, or scientific audience. Consequently when investigating into the nature of molecular binding, it is strongly encouraged to stick to particular decomposition scheme, express all definitions necessary to understand all energy terms, and keep in mind that it can be difficult if not impossible to compare results among various schemes.

The following sections present a few approaches to study the nature of XB and $\sigma$-hole interactions.

\section{$2.1 \quad \sigma$-Hole}

\subsubsection{Electrostatic Potential}

We begin with the concept that in our opinion has affected XB research most significantly the concept of $\sigma$-hole. The name " $\sigma$-hole bonding" came to apply to a class of noncovalent interactions, when it became clear that $\sigma$-hole is not related only to halogens. It might be useful to recall a quantity from classical electrostatics - the electrostatic potential (ESP). In atomic units for a set of atomic nuclei and electrons, the electrostatic potential $V(\mathbf{r})$ at spatial position $\mathbf{r}$ is defined by equation 1 . 


$$
V(\mathbf{r})=\sum_{A} \frac{Z_{A}}{\left|\mathbf{r}_{A}-\mathbf{r}\right|}-\int \frac{\rho\left(\mathbf{r}_{e}\right)}{\left|\mathbf{r}_{e}-\mathbf{r}\right|} \mathrm{d} \mathbf{r}_{e}
$$

where $Z_{A}$ is the charge of nucleus A located at position $\mathbf{r}_{A}$ and $\rho$ is the electron density at position $\mathbf{r}_{e}$. The former positive term accounts for the ESP generated by the atomic nuclei, while the latter negative term stands for the ESP of the electron cloud.

For a fixed electron density, the potential energy $E\left(\mathbf{r}_{Q}\right)$ of a probe charge $Q$ located at spatial position $\mathbf{r}_{Q}$ is then

$$
E\left(\mathbf{r}_{Q}\right)=Q V\left(\mathbf{r}_{Q}\right)
$$

It must be noted that, unlike the interaction energy or interaction energy contributions derived from any decomposition scheme, the ESP is a physical observable. The ESP can be calculated from electronic density either measured experimentally or calculated from a theoretical model. In current science, however, the distance between experiment and theory is diminishing. Without exaggeration, one can view experimental results as results of models with some (varying amount of) experimental input. Examples of ESP measurements are provided in the literature. 38139

For molecules, it is common practice to adopt the Born-Oppenheimer approximation $\frac{40}{40}$ to calculate the ESP at positions around fixed atomic nuclei. It is customary to choose the positions, but it appears advantageous to define a molecular surface and project the ESP onto it. Bader et al. proposed a surface with an electron density of 0.001 au (i.e., e/bohr ${ }^{3}$, 41 which encompasses approximately $96 \%$ of the electronic charge of a molecule. This density value became standard in calculations of molecular electrostatic potential (MEP), although other values, such as 0.0015 au and 0.002 au, have remained in use. MEPs have proven useful in understanding molecular properties and their interaction preferences, $\frac{42}{44}$ and an important finding is that the conclusions are often insensitive to the precise choice of electron density surface. 

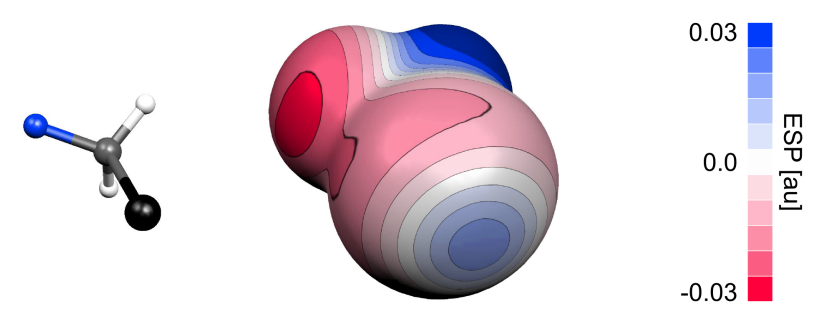

Figure 3: The ball-stick model of bromofluoromethane (left). Hydrogen is shown in white, carbon in gray, fluorine in blue, and bromine in black. On the right, the corresponding projection of a molecular electrostatic potential (ESP) in atomic units on a surface of 0.001 au electron density is shown. The blue disc on the surface in the forefront represents the $\sigma$-hole.

\subsubsection{Discovery of $\sigma$-Hole}

Analysis of MEP of halogenated methanes in 1992 revealed for the first time ${ }^{45}$ that the ESP around halogen atoms is not isotropic but exhibits regions with both positive and negative values. At that time, the positive region was found in the elongation of the $\mathrm{C}-\mathrm{X}$ covalent bond (where $\mathrm{X}=\mathrm{Cl}, \mathrm{Br}$, or I), whereas the negative region created a concentric belt around the $\mathrm{C}-\mathrm{X}$ bond (Figure 3). Based on MEPs calculated at the Hartree-Fock (HF) level, $\stackrel{46}{\text { it }}$ was possible to interpret interactions in crystal structures of halomethanes. ${ }^{47}$ In fact, close contacts of halogen atoms with electronegative moieties had been observed much earlier $\underline{48-50}$ but a satisfactory explanation had been missing.

Such a region of positive ESP was named $\sigma$-hole, 31 because the region appears in the elongation of the $\sigma$ bond of the halogen. Interestingly enough, the name was introduced 15 years after the first reference to halogen ESP anisotropy. A later review on $\sigma$-holes ${ }^{18}$ remarked on how fascinating it has been ". . .to wonder why their (XBs') contribution remained unrecognized for so long." It is even more fascinating when one realizes that the discovery of anisotropic electron density on halogens dates back to 1960s and 1970s to the studies of solid chlorine. $\underline{51,53}$

Once introduced, the term $\sigma$-hole was accepted by the XB community very quickly. 


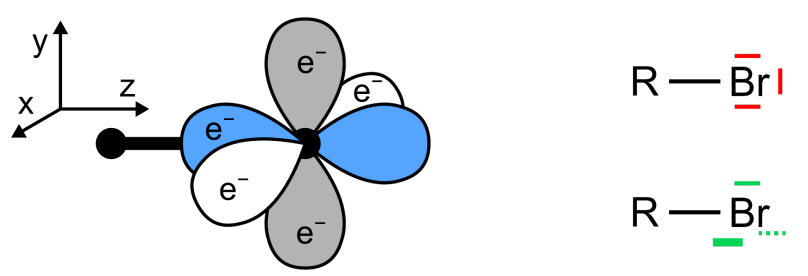

Figure 4: The orbital arrangement. Left: the $\mathrm{p}_{x}$ (white), $\mathrm{p}_{y}$ (gray), and $\mathrm{p}_{z}$ (blue) valence orbitals on a halogen atom. The electrons $\left(\mathrm{e}^{-}\right)$are localized in $\mathrm{p}_{x}, \mathrm{p}_{y}$, and the inner half of the $\mathrm{p}_{z}$ orbital. The outer half is electron-deficient, which expresses itself as a $\sigma$-hole. The remaining two valence electrons are localized in a spherically symmetric s-orbital (not drawn). Right: A typical depiction of lone electron pairs (in red) around bromine (upper structure). It evokes the misleading impression that the elongation of the $\mathrm{R}-\mathrm{Br}$ bond is electron-rich. A more correct view, which would require three dimensions (lower structure), suggests a region of electron depletion in the elongation of the $\mathrm{R}-\mathrm{Br}$ bond. The thick electron pair is in front of the plane of the paper, while the dotted one is behind (in green).

\subsection{3 $\sigma$-Holes on Halogens and Other Atoms}

Halogens were the first atoms to be shown to carry a $\sigma$-hole. Because the $\sigma$-hole is nothing but a region of ESP with certain properties, it is intimately linked to the electron density. In other words, $\sigma$-hole is a quite illustrative way of presenting electron density. Literally speaking, the electrons of a halogenated molecule simply do not like to sit on the extension of covalent bond to the halogen atom.

The concept of atomic orbitals provides an illustrative framework to understand $\sigma$-holes: the valence shell of, for example, a chlorine atom in an organic molecule has a configuration of $3 \mathrm{~s}^{2} 3 \mathrm{p}_{x}^{2} 3 \mathrm{p}_{y}^{2} 3 \mathrm{p}_{z}^{1}$, where the $\mathrm{z}$-axis coincides with the direction of the $\mathrm{C}-\mathrm{Cl}$ bond. Note that hybridization for halogens and especially for heavier halogens is not significant. The electron in the $\mathrm{p}_{z}$ orbital is mostly localized in the bond region, which results in a lack of electron density in the outer (non-involved) lobe. The electron pairs in the $\mathrm{p}_{x}$ and $\mathrm{p}_{y}$ orbitals create a negative region with a contribution of the s orbital, as depicted in Figure 4. It is intriguing to see the correspondence with a widely used depiction of halogen lone-electron pairs (Figure 4), which leads to incorrect predictions and causes the puzzling character of XBs.

One can ask if such ESP anisotropies are present on other atoms than halogens, and 
the answer is yes, definitely. It appears reasonable that the covalent bond between any two atoms affects the electron distributions of each of them. Thus, for the same reasons as halogens, also chalcogens, pnicogens, tetrels, and even aerogens (noble gases) may contain $\sigma$-holes in the elongations of their covalent bonds with neighboring atoms. $\frac{16 \mid 54}{1}$ The authors of papers on these topics advocate the use of a general name for these binding patterns: $\sigma$-hole bonding. Mostly, however, the denominations halogen (group 17 elements $-\mathrm{F}, \mathrm{Cl}$,

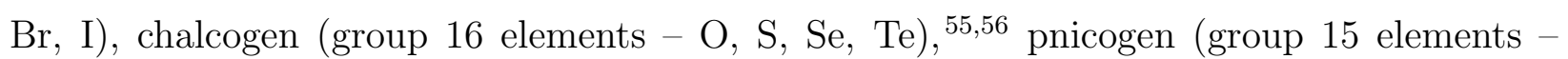
N, P, As, Sb),,$[57 \sqrt[59]{1}$ tetrel (group 14 elements - C, Si, Ge, Sn), $\underline{6061}$ and aerogen (group 18 elements - Ne, Ar, Kr, Xe) bonding $\frac{62}{6}$ are used. There is little to no evidence that the heaviest atoms of each of the groups (At, $\mathrm{Po}, \mathrm{Bi}, \mathrm{Pb}$, and $\mathrm{Rn}$, respectively) participate in similar kinds of intermolecular interactions. Halogen, chalcogen, and pnicogen bonds have one common feature: their $\sigma$-holes are easily accessible by electron donors, and the respective bonds are thus important structure-making factors. On the other hand, the $\sigma$-hole of tetrels is located in the middle of three $\mathrm{sp}^{3}$-hybridized bonds, which makes its accessibility rather low. Consequently, halogen, chalcogen, and pnicogen bonds may find broad applications in chemistry, biology, and material sciences, while potential applications of tetrel bonds are limited.

The $\sigma$-holes of group 17 elements and of other group $\mathrm{G}(\mathrm{G}=16,15$, and 14$)$ elements differ, which is connected with the systematically monovalent character of halogens, as opposed to the other elements, which can be di-, tri-, or generally polyvalent. Consequently, the halogens possess only one $\sigma$-hole, while other elements can have two, three, or even more $\sigma$-holes. The single $\sigma$-hole of halogens is located opposite to the $\mathrm{R}-\mathrm{X}$ covalent bond. This location determines the structure of halogen bonded complexes; the halogen bond tends to be linear, and this linearity is its characteristic property.

The situation with polyvalent elements is different, which can be demonstrated with group 16 elements. Figure 5 shows electrostatic potential at two sulfur-containing compounds, $\mathrm{F}_{2} \mathrm{C}=\mathrm{S}$ and $12-\mathrm{Ph}$-closo-1- $\mathrm{SB}_{11} \mathrm{H}_{20} \cdot{ }^{63}$ The sulfur atom on the first molecule is divalent, but 

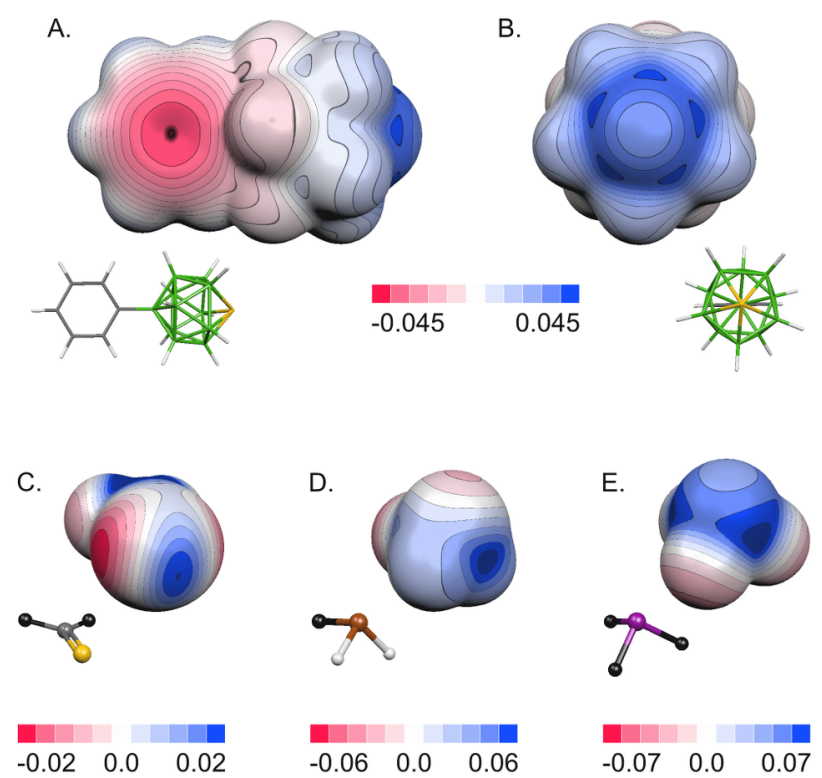

Figure 5: The electrostatic potential (ESP) projected on the surface of 0.001 au electron density. Side (A) and top (B) views of a thioborane molecule, carbonothioyl difluoride $\left(\mathrm{CSF}_{2}, \mathrm{C}\right)$, fluorophosphane $\left(\mathrm{PH}_{2} \mathrm{~F}, \mathrm{D}\right)$, and arsenic trifluoride $\left(\mathrm{AsF}_{3}, \mathrm{E}\right)$. The molecular ball-stick models are provided with the following color code: H-white, B-green, C-gray, Fblack, P-brown, S-yellow, As-purple. Note that the molecules are not proportional.

the carbon-sulfur bond is a double bond. Consequently, as with the halogens, there is only one $\sigma$-hole located on the surface of the sulfur atom. The $\sigma$-hole is clearly visible in Figure 5. with a magnitude of 0.02 au. It should be mentioned that the analog $\mathrm{H}_{2} \mathrm{C}=\mathrm{S}$ carries a negative $\sigma$-hole with a magnitude of -0.0049 au (both at the HF/cc-pVDZ level). The chalcogen bonds in complexes of the $\mathrm{F}_{2} \mathrm{C}=\mathrm{S}$ would thus be nearly linear, similar to halogen bonds.

The situation is dramatically different in thioborane (Figure 5), in which the sulfur is bound to five boron atoms and is positively charged. Interestingly, the ESP of such a molecule is highly anisotropic and shows a completely positive region on the upper part of the boron cage. The fact that pentavalent sulfur is positive is not surprising; what is surprising, however, is that the sulfur atom carries five more positive $\sigma$-holes on its side. The magnitude of these $\sigma$-holes is surprisingly high $(0.043 \mathrm{au})$; it is even higher than in the majority of halogenated systems and is comparable to the magnitude of the $\sigma$-hole on 
A.

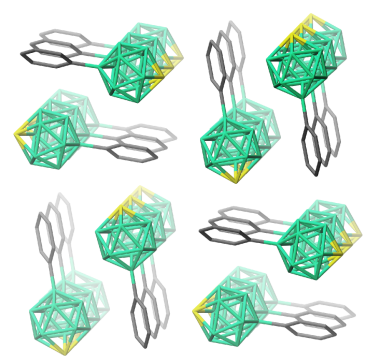

B.

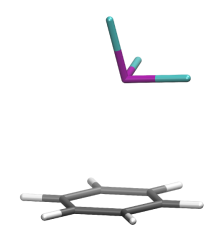

Figure 6: Crystal structures of a heterocarborane exhibiting chalcogen S... $\pi$ bond (A) (hydrogens omitted for clarity) and a benzene...AsF $\mathrm{A}_{3}$ complex with an As... $\pi$ pnicogen bond (B). The following color code is used: H-white, B-green, C-gray, F-blue, S-yellow, As-purple.

bromine in pentafluorobromobenzene. When the phenyl group in this thioborane is replaced with a chlorine atom, the magnitude of $\sigma$-hole increases $(0.049 \mathrm{au})$, which suggests that the properties of $\sigma$-holes in group 16 elements can be tuned similarly as those of group 17 elements.

$\sigma$-holes represent a structure-determining force, and the nearly linear arrangement of XB is a consequence. The $\sigma$-holes on sulfur in thioboranes are, however, not localized on top of sulfur but on its belt, which ranges from $120^{\circ}$ to $150^{\circ}$ from the $\mathrm{B}_{12}-\mathrm{S}$ axis. This reflects the covalent bonding pattern of the sulfur. Thus, the orientation of the chalcogen bonded thioboranes in not linear but bent. The $\mathrm{B}_{12}-\mathrm{S}$ axes in two 12-Ph-closo-1- $\mathrm{SB}_{11} \mathrm{H}_{20}$ molecules in the respective crystal structure are not perpendicular (Figure 6), as they should be if the $\sigma$-hole were localized on top of the sulfur atom, but have an angle of $155^{\circ}$, which is in full agreement with the prediction of non-linearity of the chalcogen bond in these thioboranes.

Figure 5 further shows the ESP of several systems containing group 15 elements. ${ }^{64}$ The situation is similar as with chalcogens discussed above. The $\sigma$-holes of trivalent pnicogens are localized on the sides of the atom, whereas the top of the pnicogen is less positive. Localization of more positive $\sigma$-holes on the belt of pnicogens will determine the structure of the respective complexes. As an example, Figure 6 shows the structure of a $\mathrm{AsF}_{3} \ldots$ benzene complex with an As... $\pi$ pnicogen bond. Assuming the $\sigma$-hole is localized on top of the As atom, the complex should have $\mathrm{C}_{3 v}$ symmetry. A non-constrained geometry optimization 
resulted, however, in a complex with lower symmetry $\left(\mathrm{C}_{1}\right)$, which is a consequence of the fact that positive $\sigma$-holes at As in the $\mathrm{AsF}_{3}$ system are localized on the belt around the atom. They preferentially interact with negative $\pi$-electron clouds of the benzene moiety. This gas-phase-optimized structure fully agrees with the experimental X-ray one. When enforcing the $\mathrm{C}_{3 v}$ structure in a similar $\mathrm{SbF}_{3} \ldots$ hexamethylbenzene complex, the respective stabilization energy decreases by $1.1 \mathrm{kcal} / \mathrm{mol}$ because the $\pi$-electron density of the hexamethylbenzene interacts with the top of the $\mathrm{Sb}$ atom, which is less positive than the respective $\sigma$-holes localized at the belt of the Sb atom.

The magnitude of the $\sigma$-holes on these pnicogens is very high, even higher than that on chalcogens, which makes the pnicogen bonds very stable. Diiodine possesses the highest magnitude among halogens investigated in this review (0.049 au); those of $\mathrm{AsCl}_{3}$ and $\mathrm{AsF}_{3}$ equal $0.061 \mathrm{au}$ and $0.080 \mathrm{au}$, respectively. Even higher magnitudes have been found for several phosphorus molecules: $\mathrm{PH}_{3}(0.025 \mathrm{au}), \mathrm{PH}_{2} \mathrm{~F}$ (0.066 au), and $\mathrm{P}(\mathrm{CN})_{3}(0.093 \mathrm{au})$.

\subsubsection{Characteristics of a $\sigma$-Hole}

Although quite late in view of the early works on $\sigma$-holes, a unified nomenclature of $\sigma$ hole properties was proposed to help in discussions of halogen bonding and $\sigma$-holes. Using a few descriptors, we aimed to understand the characteristics of $\sigma$-holes as three-dimensional spatial objects. $\frac{65 ! 67}{67}$ Because of their relative simplicity, characteristics have been defined for halogen $\sigma$-holes only, but the extension to other atoms seems straightforward. A subtle complication, however, may be seen in the more complicated topology of the chalcogen, pnicogen, and tetrel $\sigma$-holes.

The first property of interest is the maximum $\operatorname{ESP}\left(V_{\max }\right)$ located on top of the halogen atom. This property was originally proposed for hydrogens about 25 years ago,$\frac{68}{6 h i l e ~ i t s ~}$ connection with halogens appeared only one year after. ${ }^{45} V_{\max }$ is a value of ESP at the intersection of the molecular surface defined by an electron density of $0.001 \mathrm{au}^{41}$ and the elongation of the $\mathrm{R}-\mathrm{X}$ bond, where $\mathrm{X}$ stands for a halogen atom and $\mathrm{R}$ is usually carbon. 


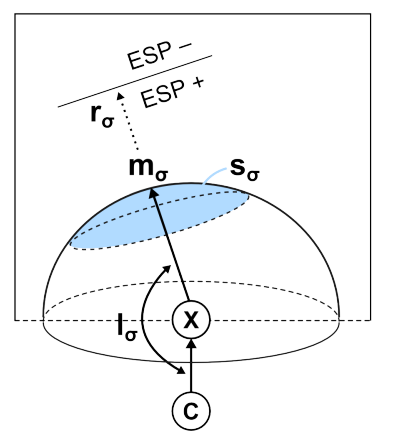

A. asymmetric

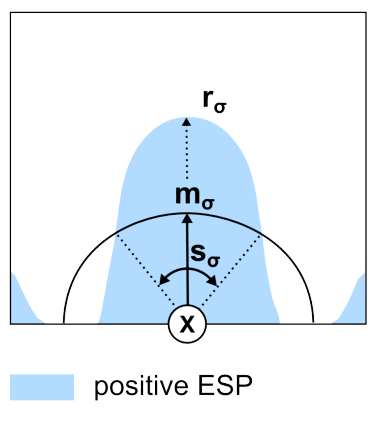

B. $\mathrm{C}_{2 v}$ symmetry

Figure 7: A scheme of $\sigma$-hole descriptors. The magnitude $\left(\mathrm{m}_{\sigma}\right)$, size $\left(\mathrm{s}_{\sigma}\right)$, linearity $\left(\mathrm{l}_{\sigma}\right)$, and range $\left(\mathrm{r}_{\sigma}\right)$ are defined in three dimensions for an asymmetric molecule (left). On the right, a two-dimensional case with $\mathrm{C}_{2 v}$ symmetry has $\mathrm{S}_{\sigma}$ defined as an angle. The size and range are meaningful only for positive $\sigma$-holes.

The value of $V_{\max }$ represents the magnitude of $\sigma$-hole $\left(\mathrm{m}_{\sigma}\right)$. However, a number of moreor-less confusing denominations of $V_{\max }$ have been also been used. Once the point with $V_{\max }-\mathrm{P}\left(V_{\max }\right)$ - has been localized on the molecular surface, the angle $\mathrm{R}-\mathrm{X}-\mathrm{P}\left(V_{\max }\right)$ can be determined. This represents the linearity of $\sigma$-hole $\left(\mathrm{l}_{\sigma}\right.$, which corresponds to angle $\phi$ in our earlier work $\left.{ }^{(66)}\right)$. Although the region of positive ESP projected on the molecular surface may have a complicated shape, the size of $\sigma$-hole $\left(\mathrm{s}_{\sigma}\right)$ can be defined as an area of the halogen MEP with the positive ESP having an approximately circular boundary (Figure 7). ${ }^{66}$ Finally, the range of $\sigma$-hole $\left(r_{\sigma}\right)$ is defined as the distance between the halogen atom and a point at which ESP changes sign from positive to negative. The range is determined in the direction $\mathrm{X}-\mathrm{P}\left(V_{\max }\right)$, and together with the size, it is meaningful only for positive $\sigma$ holes. For a molecule with $\mathrm{C}_{2 v}$ symmetry, a two-dimensional plot (Figure 7, right) provides a simpler descriptor. Instead of defining the size of $\sigma$-hole as an area, one can define it as an angle. While $\sigma$-hole magnitudes have been studied intensively, the other parameters have attracted considerably smaller attention. The parameters for selected halogenated molecules are shown in Table 1 ,

From the orbital interpretation of $\sigma$-holes (Figure 4), it arises that the lack of electron density in the outer lobe of the $\mathrm{p}_{z}$ orbital may depend on both the atomic number of the 
Table 1: The magnitude, size, linearity and range of $\sigma$-holes of selected halogenated molecules (at the CAM-B3LYP/def2-QZVP level). For hydrogenated analogues, the properties were calculated for the maximum ESP. Ph stands for phenyl.

\begin{tabular}{lrrrr}
\hline \hline & magni. [au] & size $\left[\AA^{2}\right]$ & range $[\AA]$ & linearity [deg] \\
\hline $\mathrm{Cl}_{2}$ & 0.0398 & 11.4 & $>25$ & 180.0 \\
$\mathrm{Br}_{2}$ & 0.0450 & 13.7 & $>25$ & 180.0 \\
$\mathrm{I}_{2}$ & 0.0486 & 17.0 & $>25$ & 180.0 \\
$\mathrm{H}_{2}$ & 0.0174 & 8.2 & $>25$ & 180.0 \\
$\mathrm{Cl}-\mathrm{Ph}$ & 0.0073 & 1.8 & 2.23 & 180.0 \\
$\mathrm{Br}-\mathrm{Ph}$ & 0.0152 & 4.0 & 2.78 & 180.0 \\
$\mathrm{I}-\mathrm{Ph}$ & 0.0269 & 7.6 & 4.02 & 180.0 \\
$\mathrm{H}-\mathrm{Ph}$ & 0.0230 & 7.5 & $>25$ & 180.0 \\
$\mathrm{ClCH}_{3}$ & -0.0002 & - & - & 180.0 \\
$\mathrm{BrCH}_{3}$ & 0.0089 & 2.2 & 2.39 & 180.0 \\
$\mathrm{ICH}_{3}$ & 0.0227 & 6.0 & 3.41 & 180.0 \\
$\mathrm{CH}_{4}$ & 0.0141 & 7.4 & $>25$ & 180.0 \\
$\mathrm{ClFCH}_{2}$ & 0.0108 & 3.2 & 2.52 & 178.3 \\
$\mathrm{BrFCH}_{2}$ & 0.0181 & 5.5 & 3.25 & 179.1 \\
$\mathrm{IFCH}_{2}$ & 0.0298 & 9.7 & 5.53 & 179.4 \\
$\mathrm{FCH}_{3}$ & 0.0290 & 8.4 & $>25$ & 168.3 \\
\hline \hline
\end{tabular}

halogen and its chemical environment. At first, it was not clear if the lack of electron density is manifested by a region of positive ESP or a region of negative ESP that is, however, less negative than its surroundings. The first evidence of ESP anisotropy on halogens has been reported for both of these phenomena. $\underline{45}$

It has been accepted that $\sigma$-holes are not always positive, although in early work the term $\sigma$-hole was reserved for positive ESPs only. $\frac{31}{3}$ Brinck et al. $\frac{45}{4}$ calculated the $V_{\max }$ on chlorine in $\mathrm{CH}_{3} \mathrm{Cl}$ to be -0.0033 au (at the $\mathrm{HF} / 6-31 \mathrm{G}^{*}$ level). In this case, the $\sigma$-hole is, however, less negative than the rest of the MEP on the sides of the chlorine. Negative $\sigma$-holes are often present on fluorinated or chlorinated compounds. On the other hand, a computational analysis of more than 2,500 commercially available halogenated drug-like organic molecules revealed $[\underline{66}$ that only a small fraction (less than 1\%) contains negative $\sigma$-holes; the others are positive (at the B3LYP/def2-QZVP level). A positive $\sigma$-hole is, however, not a necessary prerequisite for a molecule to participate in XB as a halogen donor. It was shown, for example, that a $\mathrm{XB}$ complex of $\mathrm{CH}_{3} \mathrm{Cl}$ with formaldehyde has a stabilization energy of $1.17 \mathrm{kcal} / \mathrm{mol}$ despite a negative $\sigma$-hole. $\frac{69}{6}$ This provides evidence 

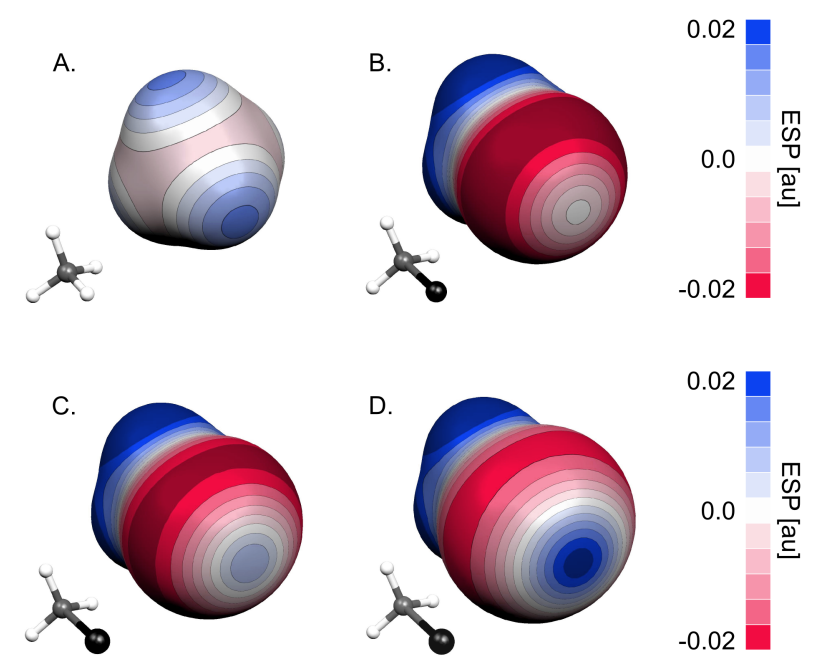

Figure 8: The electrostatic potential (ESP) projected on a surface of 0.001 au electron density of methane (A), chloromethane (B), bromomethane (C), and iodomethane (D). The ball-stick models of the molecules are provided with hydrogen in white, carbon in gray, and halogen in black. Note the same ESP scale across the surfaces with positive blues and negative reds.

that the electrostatic interaction between the $\sigma$-hole and an electron acceptor is not the only stabilizing contribution. An important part of halogen bond stabilization originates in systematically attractive dispersion energy.

There are two main factors that affect the characteristics of $\sigma$-holes: i) the nature of the halogen, defined by its atomic number, and ii) its chemical environment. Table 1 portrays both effects.

The magnitude of $\sigma$-holes increases with increasing atomic number of the halogen in the order $\mathrm{F}<\mathrm{Cl}<\mathrm{Br}<\mathrm{I}{ }^{73166}$ Figure 8 shows the differences in $\sigma$-holes for methane and halogenated methanes. The reason for these differences is the increasing polarizability and decreasing electronegativity when going from lighter to heavier halogens.

A fluorine atom has the lowest polarizability and the highest electronegativity, which earlier led to the incorrect hypothesis that fluorine does not possess a positive $\sigma$-hole and thus is unable to create halogen bonds. ${ }^{311}$ However, calculations later proved the opposite, ${ }^{70171}$ and several experiments eventually provided evidence of halogen bonds involving fluorine ${ }^{[72}$ (for review, see Mentrangolo et al. $\left.{ }^{[73}\right)$. According to a recent report, ${ }^{74}$ there exist differences in 
the properties of the electron density of fluorine and other halogens. The authors distinguish between a fluorine bond and a traditional halogen bond of $\mathrm{Cl}, \mathrm{Br}$, and $\mathrm{I}$. At the same time, however, they admit that some fluorine interactions (e.g., the $\mathrm{F}_{2} \ldots \mathrm{NH}_{3}$ interaction) are very close to the traditional halogen bond, which reflects somewhat negatively on their classification.

The positive $\sigma$-hole of fluorine has been localized on molecules including $\mathrm{F}_{2}, \mathrm{ClF}, \mathrm{BrF}_{3}$, and FCN. Apparently, the molecular fragments bound to fluorine have a common feature - they are all strongly electron-withdrawing, which seems to be a prerequisite for fluorine to possess a positive $\sigma$-hole. It is also the reason that fluorine in organic molecules (when bound to a carbon atom) typically has negative $\sigma$-holes and is unlikely to participate in XB.

It was soon recognized that $\sigma$-hole characteristics are sensitive to the chemical environment. Neighboring electron-withdrawing chemical groups (e.g., $-\mathrm{F},-\mathrm{CN})$ usually make the $\sigma$-hole more positive and bigger (compare $\mathrm{ClCH}_{3}$ and $\mathrm{ClFCH}_{2}$ in Table 1 ), whereas electron-donating groups (e.g., $-\mathrm{CH}_{3}$ ) make the $\sigma$-hole less positive and smaller. $\frac{65175176}{6}$ This is especially pronounced on halogens bound to an aromatic ring. Furthermore, Riley et al. emphasized that the $\sigma$-hole on iodobenzene has the same magnitude as the one on bromo-2,6-difluorobenzene. ${ }^{76}$ According to them, in an application in which halogen bonding of iodobenzene might not be feasible (e.g., for steric reasons), bromo-2,6-difluorobenzene could be used instead. Similarly, it has been shown that the effect of two methyl groups in meta-positions can be compensated for by a fluorine in a para-position with respect to a halogen on a benzene ring. ${ }^{65}$ This means that the $\sigma$-hole magnitudes on halobenzenes are very close to the magnitudes on 3,5-dimethyl-4-fluorohalobenzenes. Interestingly, the effect of aromatic-ring substitution on the magnitude of a halogen $\sigma$-hole is comparable to the effect on the positive ESP of a hydrogen atom in non-halogenated aromatic analogs. ${ }^{65}$

The $\sigma$-hole magnitude and size correlate well in both symmetric ${ }^{655}$ and asymmetric organic molecules. ${ }^{67}$ An interaction energy decomposition affirmed that halogens with higher $\sigma$-hole magnitude have lower local polarizabilities. ${ }^{[7]}$ An intriguing feature of halogen $\sigma$ - 
holes is that they are often surrounded by a region of negative ESP, which assigns them both electrophilic and nucleophilic character. The Protein Data Bank (PDB) contains a number of instances in which a halogen participates in a halogen bond and a hydrogen bond at the same time, and the two interactions form an angle of approximately $90^{\circ} . \underline{78}$

By tuning the size of $\sigma$-hole with neighboring chemical substitutions, it is possible to modify the ratio of the two characters. For instance, when going from bromobenzene to

para-cyano-bromobenzene, the size of $\sigma$-hole doubles, $\underline{65}$ and it can be deduced that the electrophilic character of the bromine increases at the expense of the nucleophilic one. This contrasts with the same modification of a hydrogen analogue, i.e., benzene vs. cyanobenzene. While the effect on the magnitudes is comparable for halo- and hydrogen-analogues, the effect on the sizes is dramatically different due to the missing negative belt on the hydrogen.

It must be stressed that the presence of a $\sigma$-hole is not induced by any interacting partner; it is a sole molecular property. However, the characteristics of $\sigma$-holes are indeed sensitive to their electric environment. Hennemann et al. demonstrated a notable polarization effect on $\sigma$-holes of water. .79 The polarizability in the direction of $\mathrm{R}-\mathrm{H}$ bonds,, 80 and similarly of $\mathrm{R}-\mathrm{X}$ bonds, seems to be rather simple, which turns into their strong response to an external electric field caused either by a test point charge ${ }^{79}$ or by other molecules. For instance, the polarization of the $\sigma$-hole may explain the $\mathrm{XB}$ of $\mathrm{CH}_{3} \mathrm{Cl}$, which carries a negative $\sigma$-hole in an isolated state, but a positive one induced by an interacting partner. 18181

\subsubsection{Calculations of $\sigma$-Holes}

The ESP is normally calculated from the electron density derived at the ab initio or density functional theory (DFT) level. Previous studies reflect a large ambiguity in method choice, and the basis on which one should choose the proper one remains unclear. Here, we provide a comparison of computational methods and basis sets that focuses on $\sigma$-hole properties, partially because we were unable to find a similar comparison in the literature and because we feel this comparison complements our discussion of $\sigma$-holes in this review. 
Table 2: The magnitudes of $\sigma$-hole (in atomic units) calculated at various ab initio nonempirical QM and DFT levels. The def2-QZVP basis set with the effective core potentials on iodine was used systematically. For hydrogenated analogues, the magnitude was calculated as the maximum ESP. The relative magnitudes normalized with respect to the chlorinated analogues are provided in parentheses. Ph stands for phenyl.

\begin{tabular}{|c|c|c|c|c|c|c|}
\hline & $\overline{\mathrm{HF}}$ & 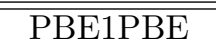 & $\overline{\overline{\text { B3LYP }}}$ & "CAM-B3LYP & $\overline{\mathrm{MP2}}$ & $\overline{\text { QCISD }}$ \\
\hline $\mathrm{Cl}_{2}$ & $0.0433(1.00)$ & $0.0403(1.00)$ & $0.0395(1.00)$ & $0.0398(1.00)$ & $0.0401(1.00)$ & $0.0395(1.00)$ \\
\hline $\mathrm{Br}_{2}$ & $0.0478(1.10)$ & $0.0456(1.13)$ & $0.0448(1.13)$ & $0.0450(1.13)$ & $0.0446(1.11)$ & $0.0445(1.13)$ \\
\hline $\mathrm{I}_{2}$ & $0.0521(1.20)$ & $0.0486(1.21)$ & $0.0475(1.20)$ & $0.0486(1.22)$ & $0.0468(1.17)$ & $0.0482(1.22)$ \\
\hline $\mathrm{H}_{2}$ & $0.0190(0.44)$ & $0.0177(0.44)$ & $0.0171(0.43)$ & $0.0174(0.44)$ & $0.0182(0.45)$ & $0.0178(0.45)$ \\
\hline $\mathrm{Cl}-\mathrm{Ph}$ & $0.0079(1.00)$ & $0.0078(1.00)$ & $0.0074(1.00)$ & $0.0073(1.00)$ & $0.0078(1.00)$ & - \\
\hline $\mathrm{Br}-\mathrm{Ph}$ & $0.0158(2.00)$ & $0.0155(1.99)$ & $0.0152(2.05)$ & $0.0152(2.08)$ & $0.0143(1.83)$ & - \\
\hline $\mathrm{I}-\mathrm{Ph}$ & $0.0290(3.67)$ & $0.0267(3.42)$ & $0.0265(3.58)$ & $0.0269(3.68)$ & $0.0236(3.03)$ & - \\
\hline $\mathrm{H}-\mathrm{Ph}$ & $0.0253(3.20)$ & $0.0238(3.05)$ & $0.0222(3.00)$ & $0.0230(3.15)$ & $0.0234(3.00)$ & - \\
\hline $\mathrm{ClFCH}_{2}$ & $0.0114(1.00)$ & $0.0105(1.00)$ & $0.0106(1.00)$ & $0.0108(1.00)$ & $0.0105(1.00)$ & $0.0111(1.00)$ \\
\hline $\mathrm{BrFCH}_{2}$ & $0.0181(1.59)$ & $0.0175(1.67)$ & $0.0177(1.67)$ & $0.0181(1.68)$ & $0.0167(1.59)$ & $0.0179(1.61)$ \\
\hline $\mathrm{IFCH}_{2}$ & $0.0317(2.78)$ & $0.0281(2.68)$ & $0.0288(2.72)$ & $0.0298(2.76)$ & $0.0263(2.50)$ & $0.0290(2.61)$ \\
\hline $\mathrm{FCH}_{3}$ & $0.0312(2.74)$ & $0.0285(2.71)$ & $0.0276(2.60)$ & $0.0290(2.69)$ & $0.0291(2.77)$ & $0.0292(2.63)$ \\
\hline
\end{tabular}

Table 2 summarizes the magnitudes of $\sigma$-holes of selected molecules calculated by several QM and DFT methods of variable complexity using the def2-QZVP basis set. ${ }^{82}$ The choice of methods was more-or-less arbitrary, and we admit that there certainly exist other DFT functionals or QM methods that would give comparable or even better results. A modest criterion here was the popularity and availability of the particular method. The column order follows the computational demands, with Hartree-Fock being the fastest and MP2 the slowest. For selected molecules, even more demanding quadratic configuration interaction with single and double excitations (QCISD) ${ }^{83}$ values were also calculated, and these might be considered as the reference values. The calculations were carried out in the Gaussian09 program package, revision D.01, ${ }^{84}$ with the help of the EMSL Basis Set Exchange database. ${ }^{85}$

The variations in the magnitudes are very small. HF gives slightly more positive ESP and the second-order Møller-Plesset perturbation theory (MP2) ${ }^{86}$ more negative values than the QCISD. All three DFT functionals are very close to the reference QCISD data. It is worth stressing that the MP2 values are noticeably less accurate than the triplet of popular DFT functionals. Importantly, Table 2 shows in parentheses the relative values normalized with respect to the chlorinated analogues. The trend that the $\sigma$-hole magnitude increases with 


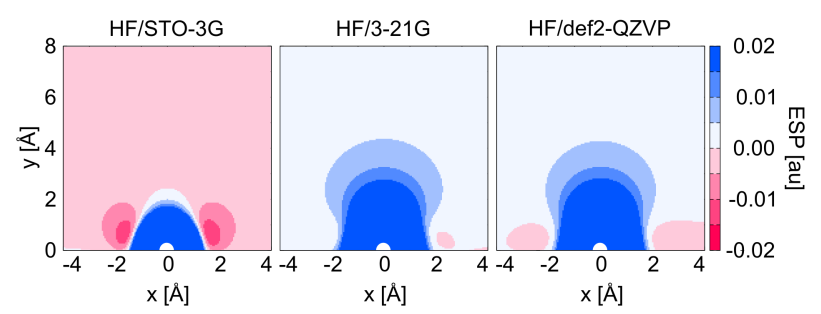

Figure 9: The projection of the electrostatic potential (ESP) in atomic units of fluorobromomethane to the plane defined by atoms F, C, and Br. The position of the bromine is represented by a white semicircle; the $\mathrm{C}-\mathrm{Br}$ bond is parallel to the $\mathrm{y}$-axis and goes to negative values. Three basis sets were used with the Hartree-Fock (HF) method. Note that the scale goes from negative reds, through neutral white, to positive blues.

increasing atomic number of the halogen is well-described, even with the least demanding method. However, when compared to QCISD, the MP2 variations of ESP within the halogens are contracted. The DFT functionals, as well as HF, perform rather well for the series of diatomics, whereas the fluoro-halogen-methanes are best described by the DFT. The differences between the functionals are minor. Overall, in terms of MEPs, none of the presented methods with the def2-QZVP basis set ${ }^{[22}$ would yield misleading results. This is contrary to XB interaction energies and geometries as discussed below in Section 2.1.6.

It is broadly believed that no $\sigma$-hole can be obtained with a minimal basis set. Figure 9 shows that this assumption is not entirely correct. The ESP maps of $\mathrm{CF}_{3} \mathrm{Br}$ were calculated at the HF level with the minimal STO-3G, small double-zeta 3-21G, and huge def2-QZVP basis sets. The ESP was projected onto the plane defined by the atoms $\mathrm{F}$, $\mathrm{C}$, and $\mathrm{Br}$. The bromine atom was located at $[0,0]$, and the $\mathrm{C}-\mathrm{Br}$ bond is parallel to the $\mathrm{y}$-axis. The anisotropy of the ESP is somehow captured by the minimal basis set, but the absolute values are significantly off. While there is a visible difference between the ESPs calculated with the minimal (39 basis functions) and double-zeta (59 basis functions) basis sets, the difference between the double-zeta and quadruple-zeta (303 basis functions) basis sets is surprisingly modest. Consequently, the minimal basis set seems to yield a $\sigma$-hole that is not very positive, or more likely negative. The use of a basis set of double-zeta or better quality is therefore strongly encouraged to describe $\sigma$-holes. 
Table 3: The magnitudes of $\sigma$-hole (in atomic units) calculated at the CAM-B3LYP level using a systematically improving basis set from the def2- series. ${ }^{82 \mid 88}$ For hydrogenated analogues, the magnitude was calculated as the maximum ESP. The relative magnitudes normalized with respect to the chlorinated analogues are provided in parentheses. $\mathrm{Ph}$ stands for phenyl.

\begin{tabular}{|c|c|c|c|c|}
\hline & "SVP & 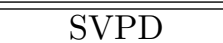 & 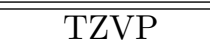 & 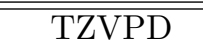 \\
\hline $\mathrm{Cl}_{2}$ & $0.0336(1.00)$ & $0.0404(1.00)$ & $0.0409(1.00)$ & $0.0407(1.00)$ \\
\hline $\mathrm{Br}_{2}$ & $0.0437(1.30)$ & 0.0466 & 0.0478 & $0.0456(1.12)$ \\
\hline $\mathrm{I}_{2}$ & $0.0526(1.57)$ & $0.0542(1.34)$ & $0.0527(1.29)$ & $0.0502(1.23)$ \\
\hline $\mathrm{H}_{2}$ & $0.0164(0.49)$ & $0.0148(0.37)$ & $0.0157(0.38)$ & $0.0167(0.41)$ \\
\hline $\mathrm{Cl}-\mathrm{Ph}$ & $0.0039(1.00)$ & $0.0065(1.00)$ & $0.0065(1.00)$ & $0.0076(1.00)$ \\
\hline $\mathrm{Br}-\mathrm{Ph}$ & $0.0153(3.92)$ & $0.0157(2.42)$ & $0.0147(2.26)$ & $0.0158(2.08)$ \\
\hline $\mathrm{I}-\mathrm{Ph}$ & $0.0306(7.85)$ & $0.0291(4.48)$ & $0.0274(4.22)$ & $0.0274(3.61)$ \\
\hline $\mathrm{H}-\mathrm{Ph}$ & $0.0185(4.74)$ & 0.0210 & $0.0214(3.29)$ & $0.0218(2.87)$ \\
\hline $\mathrm{ClFCH}_{2}$ & $0.0045(1.00)$ & $0.0127(1.00)$ & $0.0096(1.00)$ & $0.0118(1.00)$ \\
\hline $\mathrm{BrFCH}_{2}$ & $0.0156(3.47)$ & $0.0208(1.64)$ & $0.0173(1.80)$ & $0.0189(1.60)$ \\
\hline $\mathrm{IFCH}_{2}$ & $0.0313(6.96)$ & $0.0329(2.59)$ & 0.0299 (3.11) & $0.0302(2.56)$ \\
\hline $\mathrm{FCH}_{3}$ & $0.0256(5.69)$ & $0.0275(2.17)$ & $0.0274(2.85)$ & $0.0278(2.36)$ \\
\hline
\end{tabular}

The basis set dependence of the $\sigma$-hole magnitudes is given in Table 3 . The CAM-B3LYP functional was used ${ }^{[87}$ with a set of systematically improving basis sets of def2- series. ${ }^{82[88}$ The relative values normalized with respect to the chlorine analogues are provided in Table 3 in parentheses. Again, the magnitudes behave as expected; they are least positive for chlorinated compounds and most positive for the iodinated ones. Here, however, the relative magnitudes are notably basis-set dependent. A smaller basis set yields larger variations in the magnitudes. The triple-zeta basis set with diffuse functions gives practically identical relative magnitudes as the one of quadruple-zeta quality.

\subsubsection{Interaction Energies and $\sigma$-Holes}

The importance of the $\sigma$-hole for XB lies in the fact that numerous XB features can be deduced from the $\sigma$-hole properties. One of the XB features of paramount importance is its stabilization energy, the energy that is released upon formation of a halogen bond or is needed to break it. The higher the stabilization energy, the stronger the XB is said to be. Valerio et al. observed a correlation between the number of fluorines in the vicinity of the halogen atom and the stabilization energy of the halogen-bonded complex. ${ }^{89}$ At that time, 
however, such behavior was not related to the ESP.

A number of studies on $\sigma$-holes identified a relationship between the magnitude of $\sigma$ hole on the halogen donor and the stabilization energy of the respective XB. $\frac{75176190}{92}$ It was claimed that the more positive the $\sigma$-hole, the stronger interaction it creates. It was later shown that $\sigma$-hole donors other than halogens can be included into the regression analysis without disturbing the correlation, given a single electron donor. .17

The strength of any kind of noncovalent interaction depends not only on one of the partners (e.g., the halogen donor in the case of XB) but also on the other. The correlation between $\sigma$-hole magnitude and stabilization energies is considerably worse if a variety of electron donors is included. Politzer et al. have pointed out that a good correlation is obtained between the stabilization energy and the product of the maximum ESP on the halogen and the minimum ESP on the electron donor. ${ }^{92}$

Figure 10 summarizes the XB complexes investigated in our previous work. 67 The stabilization energy calculated at the best available level of theory (mostly CCSD(T)/CBS. For details, see the original work.) is plotted against the product of maximal ESP on the halogen donor and minimal ESP on the electron donor, both calculated at the PBE0/aug-cc-pVDZ level on the 0.001 au electron density isosurface. The quality of a regression fit decreases with the atomic number of the halogen involved. The correlation coefficient (R) is 0.88 for chlorinated compounds, 0.86 for brominated, and 0.69 for iodinated. The arbitrary division of the set according to the strength of the XB revealed that the stabilization energy of weaker complexes tends to correlate better with the product than that of stronger complexes. Two factors may contribute to such behavior. First, the quality of the stabilization energy, which was calculated at various levels due to the variable size of the complexes, and second, the charge-transfer character of the strongest complexes, mostly involving iodine atoms, which diverges from the presumed electrostatic origin of XB. 

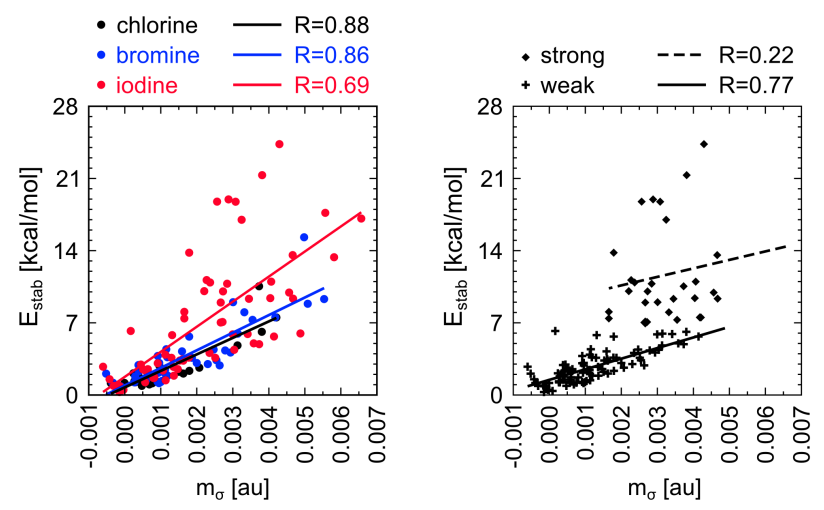

Figure 10: The dependence of the stabilization energy of XB complexes on the product of maximal ESP on the halogen donor and minimal ESP on the electron donor. The linear regression fits are provided with the correlation coefficients. The stabilization energy of $7 \mathrm{kcal} / \mathrm{mol}$ was used for an arbitrary division into classes of strong and weak XB complexes.

\subsection{Interaction Energy Decompositions}

Today, determination of accurate stabilization energies for $\sigma$-hole bonded complexes is a routine procedure, and techniques like $\operatorname{CCSD}(\mathrm{T})$ at the complete basis set (CBS) limit provide highly accurate stabilization energies for complexes with up to about 50 atoms. The total stabilization energy does not tell us, however, the leading stabilization energy term. In other words, it does not tell us which energy component (if any) is dominant. This information may be important not only for understanding the nature of this bonding but also for optimizing/maximizing the strength of the respective $\sigma$-hole bonding. Such classification of noncovalent interactions according to their nature may be practical, for example, in the area of biological sciences.

The stabilization of an $\mathrm{R}-\mathrm{X}$...Y halogen bond, where $\mathrm{X}$ is $\mathrm{Cl}$, Br, or I; R is an arbitrary chemical group (mostly carbon atom); and $\mathrm{Y}$ is an electron donor $(\mathrm{O}, \mathrm{N}, \mathrm{S}, \pi$-electrons, etc.), is explained by the existence of a positive $\sigma$-hole on top of the halogen atom.

Following the reliable CCSD(T)/CBS calculations, the stabilization energy of the most stable complex with a halogen bond from Table 4 (iodobenzene...trimethylamine) amounts to $5.8 \mathrm{kcal} / \mathrm{mol}$, and this stabilization energy is comparable to stabilization of strong hydrogen bonds. Much larger stabilization energies were, however, found (at the same theoretical 
level) for complexes of small halogen donors, including 17.1 and $15.3 \mathrm{kcal} / \mathrm{mol}$ for FI.... $\mathrm{NH}_{3}$ and $\mathrm{FBr} . . \mathrm{NH}_{3}$, respectively. ${ }^{93}$ Similarly large stabilization energies ( 8.0 and $15.0 \mathrm{kcal} / \mathrm{mol}$ ) were found (again at the same theoretical level) for crystals of large organic molecules with diiodine, 1,3-dithiole-2-thione-4-carboxyclic acid... $\mathrm{I}_{2}$ and DABCO. ... $\mathrm{I}_{2}$ complexes. ${ }^{94}$ Where do these large stabilization energies come from? Is the nature of stabilization in these complexes the same as in others?

The attraction in halogen bonded complexes was originally assigned to electrostatic attraction between the positive $\sigma$-hole and a negative electron donor, and the recent IUPAC definition of a halogen bond ${ }^{1}$ is in accord with this view. However, we recently highlighted the important role of dispersion interaction, ${ }^{9596}$ which is the attraction caused by the interaction of temporary electric multipole with an induced electric multipole.

This is understandable in the light of the fact that in any halogen bond, two atoms with high polarizability (halogen and electron donor) are located close together (closer than the sum of their van de Waals radii). This somewhat contradicts the previously mentioned definition of a halogen bond, which states, "The forces involved in the formation of the halogen bond are primarily electrostatic, but polarization, charge transfer, and dispersion contributions all play an important role." To explain this contradiction, we investigated in detail the nature of different halogen bonds, from the weakest ones with stabilization energy around a few $\mathrm{kcal} / \mathrm{mol}$ to the strongest ones with stabilization energy higher than tens of $\mathrm{kcal} / \mathrm{mol}$. We deduced the nature of the halogen bond on the basis of dominant energy terms; this means that the total stabilization energy in halogen bonded complexes should be decomposed to single energy components.

Several computational methods have been suggested to decompose the total stabilization energy. Symmetry-adapted perturbation theory (SAPT) provides a well-defined decomposition of total interaction energies into various components. With respect to halogen bonding, a number of other methods have been used to specify the nature of the bonding. After SAPT, we will discuss a few others, among which the quantum theory of atoms in molecules 
(QTAIM) is perhaps the most popular.

\subsubsection{Symmetry-Adapted Perturbation Theory}

Several energy decomposition schemes have been developed. We prefer SAPT, ${ }^{97}$ which provides a well-defined procedure for decomposing the total interaction energies into components of the first and second perturbation order. The first- and second-order perturbations composed of systematically repulsive exchange-repulsion energy, systematically attractive induction and dispersion terms and polarization/electrostatic energy. The latter can be attractive or repulsive, depending on the orientation of subsystems.

The SAPT decomposition does not recognize the electron donor - electron acceptor en-

ergy (referred to as charge-transfer), which is significant only when a good electron donor interacts with a good electron acceptor. This type of interaction occurs in halogen bonded complexes indeed, especially if the heaviest halogen (iodine) takes part. In the SAPT expansion, the charge-transfer energy is mostly covered by the induction energy. It reflects the fact that charge-transfer is only a model for physical event (electric polarization) as reported by Stone and Misquita. ${ }^{98}$ Thus it is not easy to separate the classical induction energy (multipole - induced multipole) from the charge-transfer energy. The only way to describe this systematically attractive energy term at least qualitatively is to use an alternative decomposition scheme, such as Natural Bond Orbital (NBO) analysis. ${ }^{99}$ Within this scheme, the $\mathrm{E}^{(2)}$ charge-transfer energy depends on the energy difference between localized orbitals of electron donor and acceptor as well as on the overlap of these orbitals (thus taking the geometry of the complex into account). The problem is that this energy is of different scale and cannot be directly compared with the SAPT energy terms. For a relative comparison, such as for comparison of complexes with a common electron donor and a series of halogen donors, the $\mathrm{E}^{(2)}$ energy provides a reliable relative charge-transfer estimate.

The use of the original SAPT method is limited to rather small complexes, and this was the reason for introduction of the DFT-SAPT method, 100 which allows consideration of 
complexes with up to about 40 atoms. The intramolecular treatment is conducted using the DFT, and therefore suffers from inaccurate energies of the virtual orbitals. This drawback should be corrected by performing a gradient-controlled shift procedure (see Ref. 103 ).

The total interaction energy $(\Delta E)$ is decomposed (cf, eq. 3) into first-order polarization/electrostatic $\left(E_{P o l}\right)$ and exchange-repulsion $\left(E_{E R}\right)$ terms, and second-order induction $\left(E_{I}\right)$, dispersion $\left(E_{D}\right)$, exchange-induction $\left(E_{E x-I}\right)$, and exchange-dispersion $\left(E_{E x-D}\right)$ terms. Finally, the $\delta H F$ term, which accounts for higher than second-order terms covered by the Hartree-Fock approach, is added.

$$
\Delta E=E_{P o l}+E_{E R}+E_{I}+E_{D}+E_{E x-I}+E_{E x-D}+\delta H F
$$

The exchange-induction and exchange-dispersion energies are often presented as sums with the parent induction and dispersion energies, and finally, the $\delta H F$ term is summed up with induction energy. From a brief inspection of eq. 3, we find that the charge transfer energy, which is the energy stabilizing complexes of electron donor (small ionization potential) and electron acceptor (small electron affinity), is not explicitly accounted for. It is implicitly covered by the second-order induction energy and higher-order energies covered in the $\delta H F$ term. The total induction energy thus contains not only the classical induction energy term (permanent multipole - induced multipole) but also the charge transfer (electron donor electron acceptor) energy.

An important advantage of the original SAPT method was the fact that the method was basis set superposition error (BSSE)-free. However, due to inclusion of the $\delta H F$ term calculated from the HF interaction energy (where the BSSE was considered), the resulting DFT-SAPT interaction energy is not BSSE-free.

Table 4 shows the DFT-SAPT interaction energies and their components for various electroneutral XB complexes where the type of XB is depicted. Note that dihalogen bonds of the X...X type are included in addition to standard halogen bond of the X...Y type. Throughout the study (if not otherwise mentioned), the aug-cc-pVTZ basis set was used. For 
Table 4: Decomposition of DFT-SAPT/aug-cc-pVTZ interaction energies $(\Delta E)$ of complexes with halogen bonds into electrostatic $\left(E_{P o l}\right)$, exchange-repulsion $\left(E_{E R}\right)$, dispersion $\left(E_{D}\right)$, and induction $\left(E_{I}\right)$ energy; energies in $\mathrm{kcal} / \mathrm{mol}$. Ph stands for phenyl. ${ }^{a}$ aug-cc-pVDZ basis set

\begin{tabular}{|c|c|c|c|c|c|c|c|}
\hline Complex & X-bond & $\overline{\Delta E}$ & $E_{P o l}$ & $E_{E R}$ & $E_{I}$ & $E_{D}$ & $\mathrm{X} \ldots \mathrm{Y} / \Delta \mathrm{r}$ \\
\hline $\mathrm{H}_{3} \mathrm{CCl} \ldots \mathrm{OCH}_{2}$ & Cl...O & -1.07 & -1.11 & 2.36 & -0.38 & -1.94 & $3.30 / 0.03$ \\
\hline $\mathrm{Cl}_{2} \ldots \mathrm{Cl}_{2}$ & $\mathrm{Cl} \ldots \mathrm{Cl}$ & -1.20 & -1.27 & 3.02 & -0.63 & -2.32 & $3.30 / 0.20$ \\
\hline$\left(\mathrm{CH}_{2} \mathrm{BrOH}\right)_{2}$ & Br...Br & -1.44 & -0.98 & 1.94 & -0.31 & -2.09 & $3.80 / 0.10$ \\
\hline$\left(\mathrm{CH}_{2} \mathrm{BrOH}\right)_{2}$ & Br...O & -1.56 & -2.48 & 4.30 & -0.50 & -2.88 & $3.10 / 0.27$ \\
\hline $\mathrm{H}_{3} \mathrm{CBr} \ldots \mathrm{OCH}_{2}$ & Br...O & -1.79 & -2.19 & 3.40 & -0.50 & -2.49 & $3.17 / 0.2$ \\
\hline $\mathrm{C}_{6} \mathrm{Cl}_{6} \ldots \mathrm{C}_{6} \mathrm{Cl}_{6}{ }^{a}$ & $\mathrm{Cl} \ldots \mathrm{Cl}$ & -2.10 & -1.20 & 3.57 & -0.10 & -4.37 & - \\
\hline $\mathrm{Br}_{2} \ldots \mathrm{Br}_{2}$ & $\mathrm{Br} \ldots \mathrm{Br}$ & -2.47 & -2.53 & 4.27 & -0.76 & -3.45 & $3.40 / 0.30$ \\
\hline $\mathrm{C}_{6} \mathrm{Br}_{6} \ldots \mathrm{C}_{6} \mathrm{Br}_{6}{ }^{a}$ & $\mathrm{Br} \ldots \mathrm{Br}$ & -2.90 & -2.30 & 5.80 & -0.30 & -6.10 & - \\
\hline $\mathrm{H}_{3} \mathrm{CI} \ldots \mathrm{OCH}_{2}$ & I. . .O & -2.63 & -3.53 & 4.79 & -0.94 & -2.96 & $3.21 / 0.29$ \\
\hline $\mathrm{I}_{2} \ldots \mathrm{I}_{2}$ & I...I & -3.44 & -3.61 & 5.58 & -1.09 & -4.32 & $3.70 / 0.26$ \\
\hline $\mathrm{Br}_{2} \ldots \mathrm{C}_{6} \mathrm{H}_{6}$ & Br... $\pi$ & -4.23 & -5.01 & 9.83 & -2.87 & -5.85 & - \\
\hline $\mathrm{F}_{3} \mathrm{CI} \ldots \mathrm{OCH}_{2}$ & I. ..O & -4.35 & -6.43 & 7.38 & -1.83 & -3.47 & $3.01 / 0.49$ \\
\hline $\mathrm{C}_{6}\left(\mathrm{CH}_{3}\right)_{6} \ldots \mathrm{Br}_{2}$ & Br... $\pi$ & -6.06 & -5.43 & 9.60 & -2.07 & -7.79 & - \\
\hline $\mathrm{C}_{6} \mathrm{H}_{5} \mathrm{I} \ldots \mathrm{NC}_{3} \mathrm{H}_{9}$ & I. ...N & -7.02 & -12.76 & 15.65 & -3.42 & -6.50 & $2.97 / 0.56$ \\
\hline HCN. ..BrF & Br...N & -8.24 & -14.76 & 17.22 & -5.41 & -5.30 & $2.52 / 0.88$ \\
\hline $\mathrm{H}_{3} \mathrm{~N} \ldots \mathrm{ClF}$ & $\mathrm{Cl} \ldots \mathrm{N}$ & -9.59 & -28.31 & 42.61 & -16.49 & -7.40 & $2.34 / 0.96$ \\
\hline $\mathrm{Br}_{2} \ldots \mathrm{NC}_{5} \mathrm{H}_{5}$ & Br...N & -10.89 & -19.97 & 24.15 & -7.60 & -7.47 & $2.57 / 0.83$ \\
\hline $\mathrm{H}_{3} \mathrm{~N} \ldots \mathrm{IC}_{4} \mathrm{H}_{2} \mathrm{NO}_{2}{ }^{a}$ & I. ...N & -14.25 & -25.37 & 26.16 & -8.30 & -6.74 & $2.69 / 0.84$ \\
\hline $\mathrm{I}_{2} \ldots \mathrm{NC}_{6} \mathrm{H}_{15}{ }^{a}$ & I....N & -25.20 & -46.63 & 57.85 & -22.21 & -16.34 & $2.55 / 0.95$ \\
\hline
\end{tabular}

a few of the largest complexes, the smaller aug-cc-pVDZ basis set was applied. The energy components evaluated with these two basis sets are very similar, with the exception of the dispersion term, which is underestimated by $10-15 \%$ with the smaller basis set. Complexes are ordered by increasing stabilization; the stabilization energy of the strongest complexes is substantial, more than $25 \mathrm{kcal} / \mathrm{mol}$. A stabilization energy of $7 \mathrm{kcal} / \mathrm{mol}$ was considered (arbitrarily) as the border between weak and medium/strong halogen bonded complexes. The same limit was considered in our previous study, ${ }^{67}$ in which we investigated 129 XB complexes: 38 with stabilization energies larger than $7 \mathrm{kcal} / \mathrm{mol}$ and 91 with stabilization energies between 0.3 and $7 \mathrm{kcal} / \mathrm{mol}$. In the following paragraphs, the composition of total interaction energy and complex properties will be discussed separately for the two classes of XB complexes.

We start with the X...Y intermolecular distances, which for all XB complexes (Table 4 are shorter than the sum of the respective van der Waals radii (vdW distance), which

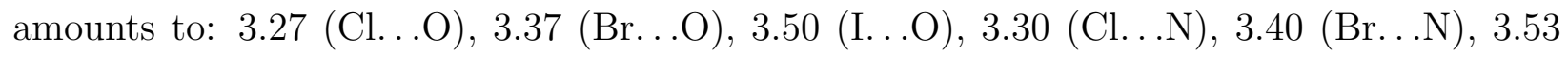


(I . . N), 3.55 (Cl. . S), 3.65 (Br. . S), 3.78 (I . .S), 2.70 (F . .F), 3.50 (Cl . .Cl), 3.70 (Br. . . Br), and 3.96 (I. . .I) with all values in $\AA$. The shortest distance of $2.34 \AA$ was found for $\mathrm{H}_{3} \mathrm{~N}$. . .ClF complexes, and the contraction of the vdW distance was substantial, approximately $1 \AA$. For the six strongest complexes, the intermolecular distance was shorter than $3 \AA$, and the respective contraction was larger than $0.5 \AA$. For five of these six complexes, the contraction was even larger than $0.8 \AA$. Large contraction of the interatomic distance is, as expected, connected with a very large repulsion from the exchange-repulsion term.

By investigating the nature of stabilization in the group of medium/strong complexes, we found that the polarization/electrostatic energy is mostly dominant. Very large (attractive) polarization energies are connected with very large magnitudes of the $\sigma$-holes of the respective halogen donors. However, because the value of polarization energy and magnitude of the $\sigma$ hole do not correlate, it is clear that other attractive electrostatic components (e.g., the dipole - dipole electrostatic term) also contribute. The other attractive energy terms, induction and dispersion, are large but contribute less than half of the polarization term. Surprisingly, in five of six cases, the induction energy was the second largest stabilization term. In these cases, the stabilization clearly stems from the charge-transfer interaction and not from the standard induction term of the multipole - induced multipole type. Diiodine and other dihalogens such as dibromine and ClF are very good electron acceptors, which can be seen from their lowest unoccupied molecular orbital (LUMO) energies: $-0.144,-0.140$, and -0.127 $\mathrm{au}$, respectively. Some organic compounds containing halogens, like $\mathrm{IC}_{4} \mathrm{H}_{2} \mathrm{O}_{2} \mathrm{~N}$, also are very good electron acceptors and possess very low LUMO energy (-0.106 au).

The second group of XB complexes consists of 13 complexes with stabilization energy between 1.07 and $6.06 \mathrm{kcal} / \mathrm{mol}$ (Table 4). In these cases, the XB is systematically longer than those in the first group, and is larger than $3 \AA$. The contractions of XB distance with regard to the van der Waals radii are lower and do not exceed $0.5 \AA$; within the weakest complexes (below $2 \mathrm{kcal} / \mathrm{mol}$ ), it is less than $0.2 \AA$. The dominant stabilization term is, surprisingly, the dispersion energy. Only in two of 13 cases is the polarization term the largest 
in magnitude. In these two cases, the halogen donor molecule contains iodine (iodomethane and trifluoroiodomethane), and the magnitude of the $\sigma$-hole is rather high (0.022 and 0.049 $\mathrm{au}$ ). In the remaining 11 complexes, the dominant dispersion energy is followed by the polarization and induction terms. The induction energy is typically lower than $1 \mathrm{kcal} / \mathrm{mol}$.

The contribution of the SAPT-like dispersion energy to the stability of halogen-bonded complexes is surprising, and it is much larger than that of similar hydrogen-bonded (HB) complexes. This can be easily explained by the fact that in XB two heavy atoms (the halogen and the electron donor) with high polarizabilities are in close contact (see intermolecular distances in Table 4), whereas in the case of HB, only the light hydrogen and the electron donor are in close contact. In the XB complexes, the dispersion energy of the contact atom pair contributes as much as $40 \%$ of the total dispersion energy between halogen donor and electron acceptor. ${ }^{67}$ This significant contribution is related to the rather short X. . .electron donor distance. Two mutually consistent views may explain this low interatomic distance: i) the attractive interaction between the positive $\sigma$-hole on the halogen and the negative electron donor and ii) the lower exchange-repulsion in the direction of the XB between the two subsystems, which is due to polar flattening. 109

The first point is intuitive - the absence of a positive $\sigma$-hole, the Coulomb repulsion would separate both subsystems and the intermolecular distance would thus be larger; due to the $\mathrm{r}^{-6}$ dependence, the dispersion energy would decrease even more significantly. The second point is slightly extraordinary and originates in a non-spherical electron density around the halogen atom covalently bound to another atom. This so-called polar flattening (Figure 11) is a consequence of the fact that a halogen atom is covalently bound to another atom. ${ }^{53} \mathrm{We}$ should note that the effect of polar flattening is not limited to a halogen atom covalently bound to another atom, but exists for any atom that is covalently bound to another one. This effect is due to a flattening of the spherical electron density of an isolated atom in the direction of a covalent bond, and it is most pronounced in the case of monovalent atoms.

It can be argued, however, that the polar flattening and $\sigma$-hole are two ways of expressing 


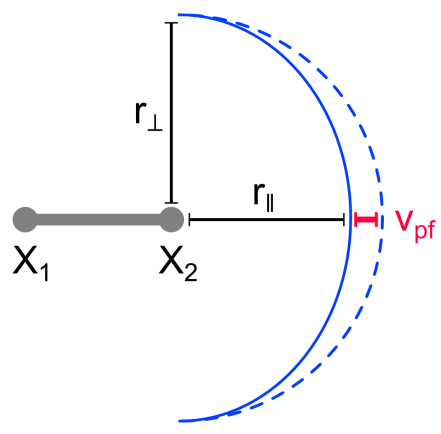

Figure 11: A scheme of polar flattening of an $\mathrm{X}_{1}-\mathrm{X}_{2}$ dihalogen molecule. The electron isodensity surface is not spherically symmetric (dashed blue line), but flattened in the elongation of the covalent bond (solid blue line). The value of such flattening $\left(v_{p f}=r_{\perp}-r_{\|}\right)$is dependent on the electron density. Figure adapted with permission from Ref ${ }^{110}$ Copyright 2015 American Chemical Society.

the same - the electronic situation around a molecule - in the former case at the level of electron density, whereas at the latter case at the level of electrostatic potential. Then one must accept that there is no causality between polar flattening and $\sigma$-hole.

Recently, we investigated ${ }^{[10}$ the role of polar flattening in several model halogen-bonded complexes and concluded that it is not negligible and reaches $0.1-0.2 \AA$ and $10-15 \%$ for absolute and relative values of intermolecular distance, respectively. We compared the real situations with a hypothetical ones, where the halogens were spherically symmetric. These geometry changes would induce stabilization energy changes that would be relatively small due to compensation of attractive and repulsive energy terms. The shortening of the intermolecular distance caused by polar flattening would be responsible for a relative increase in stabilization energy up to $15 \%$.

In summary, the characteristic properties of $\mathrm{XB}$ complexes are due to the concerted action of polarization/electrostatic, dispersion, and induction energy terms in the sense of SAPT decomposition. While for the strongest XB complexes $(>7 \mathrm{kcal} / \mathrm{mol})$, the dominant polarization energy is followed by induction (here charge-transfer is significant) and dispersion terms, for weak and moderate XB complexes $(1-7 \mathrm{kcal} / \mathrm{mol})$, the dispersion energy is dominant. Very similar conclusions were drawn from the whole set of $129 \mathrm{XB}$ complexes investigated previously. $\frac{67}{67}$ Thus, we conclude that in our opinion the IUPAC definition of 
a halogen bond ("The forces involved in the formation of the halogen bond are primarily electrostatic, but polarization, charge transfer, and dispersion contributions all play an important role." $)^{1}$ is not sufficiently accurate and does not satisfactorily and fully describe the unique phenomenon of XB.

In the previous part, we investigated the nature of halogen bonds in different complexes with halogen and dihalogen bonds. Next, we extend our efforts to complexes with chalcogen and pnicogen bonds. Bleiholder et al. performed an early SAPT analysis of chalcogenbonded complexes ${ }^{111}$ before the terms "chalcogen bond" and " $\sigma$-hole bond" entered into use. On a set of complexes containing homoatomic contacts of $\mathrm{O}, \mathrm{S}, \mathrm{Se}$, and Te, they found that in terms of SAPT components, the induction and dispersion energies are the leading terms in most cases. They noticed that the competition between chalcogen bonding, hydrogen bonding, and other interactions is less equable with increasing atomic number of the chalcogen, i.e., Te-complexes prefer chalcogen bonds over hydrogen bonds more strongly than O- or S-complexes.

A SAPT comparison of hydrogen, pnicogen, chalcogen, and halogen bonds in four complexes containing $\mathrm{NH}_{3}$ revealed that the polarization and induction terms are dominant. 112 The role of dispersion was claimed to be rather low, but not negligible. It must be noted that the stabilization energies of these complexes are between 6 and $11.6 \mathrm{kcal} / \mathrm{mol}$ (at the MP2/aug-cc-pVDZ level), so the importance of polarization and induction terms is in line with the DFT-SAPT decompositions of XB complexes.

Here, we continue with an analysis similar to the XB one based on DFT-SAPT calculations. Table 5 summarizes the DFT-SAPT energies for selected complexes with a chalcogen or pnicogen bond. The chalcogen-bonded thioborane dimer shows a very large stabilization energy, in which the dispersion contribution is clearly dominant. However, the electrostatic term is also substantial, and stabilization of this structure is largely due to these two attraction terms. The stacking structure of the dimer is less stable due to a less attractive electrostatic term; note that the dispersion energy is almost as important as it is in the first 
Table 5: Decomposition of DFT-SAPT/aug-cc-pVDZ interaction energies $(\Delta E)$ of complexes with chalcogen and pnicogen bonds into electrostatic $\left(E_{P o l}\right)$, exchange-repulsion $\left(E_{E R}\right)$, dispersion $\left(E_{D}\right)$, and induction $\left(E_{I}\right)$ energy; energies in kcal/mol. Ph stands for phenyl.

\begin{tabular}{llccccc}
\hline \hline Complex & type of bond & $\Delta E$ & $E_{P o l}$ & $E_{E R}$ & $E_{D}$ & $E_{I}$ \\
\hline $\mathrm{Ph}_{-} \mathrm{SB}_{11}$ dimer & B-S..$\pi$ & -8.8 & -5.8 & 10.8 & -12.0 & -2.0 \\
$\mathrm{Ph}_{-} \mathrm{SB}_{11}$ dimer & Stacking & -6.6 & -2.4 & 9.4 & -14.8 & -0.8 \\
$\mathrm{C}_{6} \mathrm{H}_{6} \ldots \mathrm{AsF}_{3}$ & As... & -2.0 & -1.6 & 4.6 & -4.2 & -0.8 \\
$\mathrm{C}_{6} \mathrm{H}_{6} \ldots \mathrm{AsCl}_{3}$ & $\mathrm{As} \ldots \pi$ & -2.9 & -2.2 & 6.4 & -6.2 & -0.9 \\
$\mathrm{C}_{6} \mathrm{H}_{6} \ldots \mathrm{SbF}_{3}$ & $\mathrm{Sb} \ldots \pi$ & -6.9 & -5.8 & 5.4 & -4.4 & -2.0 \\
$\mathrm{C}_{6} \mathrm{H}_{6} \ldots \mathrm{SbCl}_{3}$ & $\mathrm{Sb} \ldots \pi$ & -6.9 & -6.1 & 7.1 & -5.9 & -2.0 \\
$\mathrm{C}_{6} \mathrm{~F}_{6} \ldots \mathrm{AsF}_{3}$ & $\mathrm{As} \ldots \pi$ & -2.0 & -1.6 & 4.6 & -4.2 & -0.8 \\
$\mathrm{C}_{6} \mathrm{~F}_{6} \ldots \mathrm{AsCl}_{3}$ & $\mathrm{As} \ldots \pi$ & -2.9 & -2.2 & 6.4 & -6.2 & -0.9 \\
$\mathrm{C}_{6} \mathrm{~F}_{6} \ldots \mathrm{SbF}_{3}$ & $\mathrm{Sb} \ldots \pi$ & -3.1 & -3.2 & 4.6 & -3.9 & -0.6 \\
$\mathrm{C}_{6} \mathrm{~F}_{6} \ldots \mathrm{SbCl}_{3}$ & $\mathrm{Sb} \ldots \pi$ & -3.3 & -3.2 & 6.0 & -5.3 & -0.8 \\
\hline \hline
\end{tabular}

structure. Similar conclusions confirming a dominant role of dispersion and, to lesser extent, electrostatic energy were found for complexes of neutral closo-heteroboranes possessing chalcogen or pnicogen bonds. ${ }^{113}$ All remaining complexes in Table 5 exhibit pnicogen bonds, and among them, the benzene...SbX 3 complexes are the most stable. Their exceptional stabilization is due to the concerted action of electrostatic and dispersion energy. For all other complexes with a pnicogen bond, the dispersion energy is clearly dominant.

Replacing benzene with another electron donor (e.g., $\mathrm{NH}_{3}$ ) results in even stronger pnicogen bonds. The situation with other pnicogen bonds might be different. Recently, Setiawan et al. ${ }^{114}$ published an extended study on pnicogen bonds in complexes involving the group Va elements N, P, and As. They investigated a set of 36 homo- (E...E) and heterodimers (E...E'), where E stands for a group 15 element, at the $\omega$ B97X-D/aug-cc-pVTZ level. They showed that a subset of 10 dimers was mainly stabilized by pnicogen covalent bonding characterized by significant charge transfer (CT) from a donor lone pair orbital to the acceptor $\sigma^{*}$ orbital (through-space anomeric effect), while all the other dimers were stabilized by electrostatic interactions. These conclusions were, however, not based on SAPT calculations.

For the pnicogen set, we performed DFT-SAPT calculations similar to those shown in Tables 4 and 5. For the 10 dimers shown to be stabilized by pnicogen covalent bonding, the polarization/electrostatic energy was systematically dominant; the induction energy was 
important for all these complexes, but in all cases it was comparable to the dispersion energy. The polarization/electrostatic energy was dominant in $65 \%$ and dispersion energy in $35 \%$ of the remaining complexes. Evidently, for pnicogen complexes, the polarization/electrostatic energy is mostly dominant, followed by dispersion and induction energies. The important role of polarization/electrostatic energy is related to the high magnitudes of the $\sigma$-hole for these systems. The $\mathrm{H}_{3} \mathrm{~N} \ldots \mathrm{P}(\mathrm{CN})_{3}$ complex had the highest DFT-SAPT stabilization energy $(8.5 \mathrm{kcal} / \mathrm{mol})$ of all complexes investigated. Its polarization/electrostatic energy ($19.9 \mathrm{kcal} / \mathrm{mol})$ was followed by induction and dispersion energies $(-7.2$ and $-6.2 \mathrm{kcal} / \mathrm{mol}$, respectively). As mentioned above, the magnitude of the $\sigma$-hole for $\mathrm{P}(\mathrm{CN})_{3}$ is very high (0.093 au), likely the highest among all complexes mentioned in this review.

For the above-mentioned small model complexes with pnicogen bonds, the polarization/electrostatic energy is dominant. To elucidate the role of polarization/electrostatic and dispersion energies, further investigation of a more extended set of larger complexes with pnicogen bonds is needed. From the Cambridge Structural Database, we selected 95 structures exhibiting close contacts between pnicogens (As and Sb) and electron donors ( $\pi$-electrons, N, O, S, Se, Te, Cl, I). The stabilization energies of all of these complexes were determined at the DFT-D3/BLYP/def2-TZVPP level. A comparison of the dispersion energy with the total stabilization energy can shed light on the relative role of the two contributions.

The first results were encouraging. For 10 As. . $\pi$ and 30 Sb... $\pi$ complexes, the dispersion energy is systematically larger than the electrostatic one. The respective stabilization energies fall within a range between 4.9 and $16 \mathrm{kcal} / \mathrm{mol}$. For 9 As...X and $46 \mathrm{Sb}$..X complexes, the dispersion energy is larger in 3 and 17 cases, and the respective stabilization energies were within the $3.7-32.8 \mathrm{kcal} / \mathrm{mol}$ range. Altogether, from 95 complexes with a pnicogen bond, the dispersion energy was dominant in 60 cases, while the electrostatic energy was dominant only in 35 cases. Further systematic studies based on DFT-SAPT calculations are required for final estimation of the role of polarization/electrostatic and dis- 
persion energies, but the preliminary results show that the dispersion energy seems to be dominant in the majority of complexes with pnicogen bonds.

In conclusion, the characteristic properties of halogen, chalcogen, and pnicogen bonding are due to the concerted action of polarization/electrostatic and dispersion energy terms in the SAPT sense. In the case of halogen bonded complexes with a heavier halogen, mainly iodine, the induction/charge-transfer energy is also significant. The structure of $\sigma$-hole complexes is due to specific polarization/electrostatic energy while the stability is due to nonspecific dispersion energy, an important portion of which originates in the interaction of the atom that carries the $\sigma$-hole and the electron donor.

\subsubsection{Other Schemes}

$\mathrm{NBO}^{99}$ analysis is one way to estimate charge transfer contribution. As mentioned above, the values tend to be too high, so this should serve as a qualitative approach. For example, it was shown that the charge transfer in complexes in which fluorine stands for an electron acceptor is rather low except for the $\mathrm{F}_{2} \ldots \mathrm{NH}_{3}$ complex. ${ }^{70}$ This contrasts with complexes of bromobenzene with several electron donors, in which the charge transfer was found to be significant. ${ }^{90}$ The stabilization of various $\mathrm{X}_{2}$ complexes has been studied using NBO.115/116 $\mathrm{X}_{2}$ molecules are intrinsically different from $\mathrm{N}_{2}$ (dinitrogen), which has consequences for their binding preferences.

NBO population analysis was used in the seminal paper of Clark et al., ${ }^{31}$ who demonstrated on $\mathrm{CF}_{3} \mathrm{X}(\mathrm{X}=\mathrm{F}, \mathrm{Cl}, \mathrm{Br}$ or $\mathrm{I})$ that the level of hybridization of the valence-shell $\mathrm{s}$ and p orbitals is very low (about 10\%) for heavier halogens, but notably higher for fluorine. Also, the contribution of the valence p orbital to the bonding $\sigma_{C-X}$ orbital is significant, which justifies the intuitive view of the valence-shell configuration of $\mathrm{s}^{2} \mathrm{p}_{x}^{2} \mathrm{p}_{y}^{2} \mathrm{p}_{z}^{1}$ (in which the Z-axis coincides with the $\mathrm{C}-\mathrm{X}$ bond).

In the context of noncovalent binding, the quantum theory of atoms and molecules $\underline{41117}$

has become particularly popular despite some controversial conclusions based on it. $\frac{118 \mid 119}{10}$ 
QTAIM stems from topological analysis of the electron density $\rho$, its gradient $\nabla \rho$, and Laplacian $\Delta \rho$ (sometimes denoted $\nabla^{2} \rho$ ). The noteworthy feature of the electron density is that, in contrast to the $4 \mathrm{~N}$-dimensional wave function, it is only three-dimensional.

Generally, bond critical points (BCPs) are identified for halogen bonds. The electron density at BCPs represents a meaningful measure of the XB strength. ${ }^{90}$ Halogen bonding is an interaction between a $(3,-1)$ type critical point $(\mathrm{CP})$ - a hole on the electron acceptor - and a $(3,-3) \mathrm{CP}$ - a lump on the electron donor. $\frac{120}{1 n}$ terms of QTAIM, a number of similarities have been found between halogen, dihalogen, and hydrogen bonding. ${ }^{121}$ QTAIM was also used to justify the existence of XB in fluorinated compounds. ${ }^{74}$

Let us finalize this section by listing several studies of halogen and $\sigma$-hole bonding using less common quantum chemical approaches: PIXEL scheme, $\frac{122}{1}$ intermolecular perturbation theory, ${ }^{123}$ Kohn-Sham molecular orbital analysis, ${ }^{124}$ Fukui function, ${ }^{931125}$ noncovalent inter-

action plot, ${ }^{931126}$ energy decomposition analysis (EDA), and Voronoi deformation density. ${ }^{125}$ All of these bring certain insights into halogen bonding, but mutual comparison is not possible due to natural differences and nomenclature of the schemes. Some of the methods are reviewed in more detail in Ref. .15

\section{Computational Methods}

\subsection{Benchmark Calculations and Performance of Various Compu- tational Methods}

Benchmark stabilization energies of halogen-bonded complexes can be obtained similarly to those of other noncovalently bound molecular clusters by exploiting the coupled cluster (CC) theory. The CC theory, introduced to quantum chemistry by the Czech physicist Jiří Č́žzek more than a half-century ago, ${ }^{127128}$ relies on the exponential formulation of the wave operator and its expansion into cluster excitation operators. One of the most important features of the $\mathrm{CC}$ theory is the fact that it is systematically improvable upon inclusion of a 
higher excitation operator. ${ }^{129}$ Accurate energies are, however, obtained only if a sufficiently large AO basis set is adopted. Then, the following hierarchy of accuracy is (mostly) valid:

$\operatorname{CCSD}<\operatorname{CCSD}(\mathrm{T})<\operatorname{CCSDT}<\operatorname{CCSDT}(\mathrm{Q})<\operatorname{CCSDTQ}<\ldots<\mathrm{FCI}$

where S, D, T, and Q stand for single, double, triple, and quadruple electron excitation, respectively. Placing a letter in parentheses indicates that a non-iterative perturbative treatment takes place, while in other cases fully iterative treatment is adopted. FCI stands for the full configuration interaction, and the respective energy represents the most accurate value that can be obtained within the basis set used. $\frac{130}{10}$ The interaction energy obtained at the FCI level thus represents the benchmark value that other methods can only approach. Triple excitations play an important role in covalent and noncovalent interactions, and thus the CCSD $(\mathrm{T})$ scheme represents a widely applicable method. The CCSD(T) method, characterized by iterative inclusion of the single and double excitations with noniterative, perturbative inclusion of triple (4th order) and single (5th order) excitations, is especially successful for ground state energies and the calculation of properties for systems with single-reference character. As demonstrated, $\stackrel{128}{ } \operatorname{CSD}(\mathrm{T})$ is the "golden standard" of CC-approximations due to its outstanding accuracy-to-computational-costs ratio.

As mentioned above, accurate results are only obtained if an extended AO basis set or a complete basis set (CBS) limit is applied. The CBS limit is now commonly determined by an extrapolation technique, and Helgaker 2- or 3-point extrapolation 131 is frequently adopted. The 2-point extrapolation form contains one parameter obtained from previous calculations. The 3-point one is parameter-free, and this technique was used in determination of stabilization energies in the A24 dataset, $\stackrel{132}{ }$ which contains 24 small noncovalent complexes of different types (hydrogen-bonded complexes, dispersion-bound complexes, etc.).

Interaction energies in this dataset were determined directly as the difference between total CCSD(T) energy of the complex and the sum of subsystem energies. Three systematically improved AO basis sets were used: aug-cc-pVTZ, aug-cc-pVQZ, and aug-cc-pV5Z. Augmented basis sets were used, and the smallest basis set was of triple-zeta quality. The 
function counterpoise procedure of Boys and Bernardi ${ }^{133}$ was used throughout the study. The obtained energies represent a highly accurate benchmark that serves for testing and/or parameterization of lower-level methods and techniques that allow treatment of more extended noncovalently bound complexes. The use of the $\operatorname{CCSD}(\mathrm{T})$ with pentuple-zeta basis set is limited to complexes containing no more than five heavy atoms. For larger complexes, the calculation procedure should be modified, and instead of direct determination of interaction energy from total $\operatorname{CCSD}(\mathrm{T})$ energies, a composite scheme ${ }^{134}$ can be used successfully.

The applicability of the composite scheme (eq. 4) is based on the different basis set size dependence of the energy components of total $\operatorname{CCSD}(\mathrm{T})$ energy, i.e., the HF, MP2, and $\triangle \mathrm{CCSD}(\mathrm{T})$ energies. HF energy, which can be calculated relatively easily, depends least on the basis set size, and an extended basis set (e.g., aug-cc-pVQZ) can be used. MP2 energy is extrapolated to the CBS limit, and an aug-cc-pVDZ $\rightarrow$ aug-cc-pVTZ or, preferentially, an aug-cc-pVTZ $\rightarrow$ aug-cc-pVQZ scheme can be utilized. Finally, the computationally most difficult term, the $\triangle \mathrm{CCSD}(\mathrm{T})$ one, calculated as the difference between $\mathrm{CCSD}(\mathrm{T})$ and MP2 energies, is determined in the smaller basis set. The lesser dependence of the $\Delta \mathrm{CCSD}(\mathrm{T})$ term is due to the similar dependence of MP2 and CCSD(T) energies on the size of the basis set. The size of the basis set used to compute the $\Delta \mathrm{CCSD}(\mathrm{T})$ term has a large effect on the quality of the final energies, and usually the aug-cc-pVDZ or, preferentially, the aug-cc-pVTZ basis set are utilized (Equation 4).

$$
\begin{aligned}
\Delta E(C C S D(T) / C B S) & \approx \Delta E(H F / a u g-c c-p V Q Z)+\Delta E(M P 2 / C B S)+ \\
& +[\Delta E(C C S D(T) / a u g-c c-p V D Z)-\Delta E(M P 2 / a u g-c c-p V D Z)]
\end{aligned}
$$

The present scheme allows treatment of much larger complexes (containing up to $\sim 40$ atoms) than the previous one and still provides highly accurate values. The average unsigned error for these two approaches for the A24 dataset ${ }^{132}$ is only $1.04 \%$. This excellent accuracy is, however, the result of error compensation, and other combinations of basis sets provide 
larger errors.

The performance of computational methods has been tested on several data sets of halogenated complexes. The importance of going beyond MP2 for halogen-bonded complexes was recognized rather early in the work of Karpfen, $\frac{137}{17}$ who calculated geometries and stabilization energies of complexes of dihalogens with $\mathrm{NH}_{3}$ at the $\operatorname{CCSD}(\mathrm{T})$ level. MP2 cannot provide benchmark stabilization energies; thus, its use for assessing the reliability of other $a b$ initio and DFT methods cannot be recommended. More precisely, the quality of MP2 has been tested, and for some applications it may provide sufficiently accurate results. These are, however, not of a benchmark quality. Consequently, we limit our attention to studies in which the CCSD $(\mathrm{T})$, preferentially with a basis set extrapolated to the CBS limit, was used.

The X40 data set from our laboratory ${ }^{69}$ focuses on XB and non-XB complexes of halogenated molecules and thus acts as a non-biased set for general purposes. In this regard, the

X40 data set is analogous to a more general S66 data set.138 The X40 data set has mainly been used to characterize the reliability of ab initio non-empirical QM methods, but also for parameterization of lower-level computational methods. On the other hand, the XB18 and XB51 data sets developed by Kozuch and Martin ${ }^{93}$ contain XB complexes only. In addition to using ab initio methods, they heavily inspected the reliability of density functional schemes, thus nicely complementing our X40. Furthermore, the XB18 set has been used to assess XB geometries.

Other $\sigma$-hole interactions have not been investigated as extensively, apart from an assessment of DFT functionals by Bauzá et al.139

\subsubsection{X40 Data Set}

Benchmark 10-point dissociation curves were determined for the representative set of model halogen-bonded complexes shown in Figure 12. They represent a subset of a broader series of halogen-containing complexes called X40. $\underline{69}$ The complex geometries were optimized at the counterpoise-corrected MP2/cc-pVTZ level with the effective core potentials on Br and I 

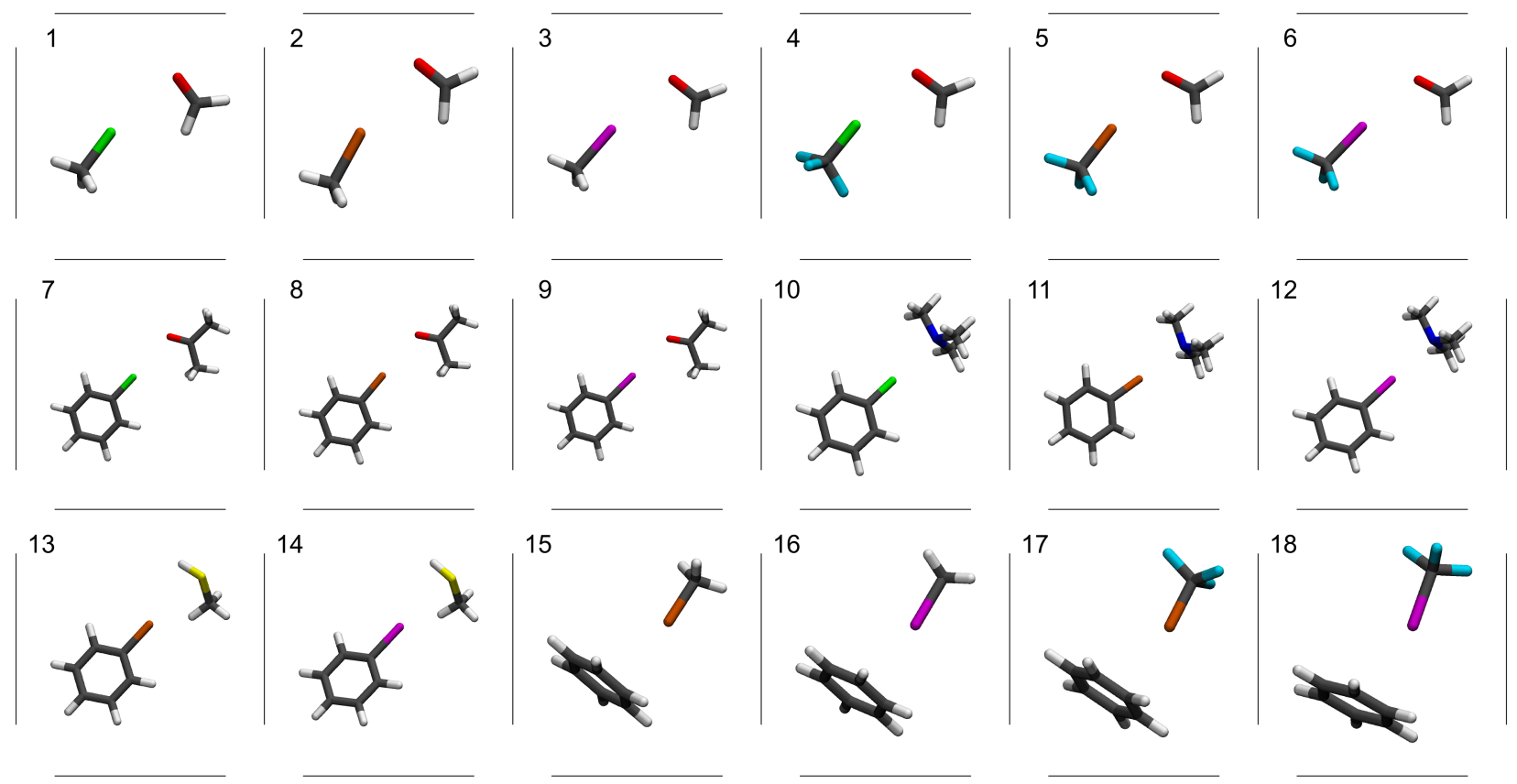

Figure 12: 18 halogen-bonded complexes from the X40 set. ${ }^{69}$ Color code: H-red, N-navy, C-gray, O-red, F-cyan, S-yellow, Cl-green, Br-orange, I-purple.

atoms. As previously stated, ${ }^{69}$ this "basis set was found to provide the most favorable error compensation when used with MP2, and the resulting geometries are in good agreement with the $C C S D(T) / C B S$ reference." $[140$ From the optimized geometries, the dissociation curves were constructed by multiplying the intermolecular distance by factors between 0.8 and 2.0, while keeping the mutual orientation of the monomers fixed.

The stabilization energies were determined at the HF and $\triangle \operatorname{CCSD}(\mathrm{T})$ levels with the aug-cc-pVQZ and aug-cc-pVDZ basis sets, respectively, and the MP2 energy was extrapolated using the aug-cc-pVTZ $\rightarrow$ aug-cc-pVQZ scheme. To test the accuracy of this setup, we calculated interaction energies also at the higher theoretical level. Specifically, the HF and MP2 energies were calculated at the same level, but the critical $\Delta \mathrm{CCSD}(\mathrm{T})$ term was determined with the aug-cc-pVTZ basis set (for hydrogens, only the cc-pVTZ basis set was used). The improvement in accuracy over the previous level was negligible, with a root-mean-square difference of $0.06 \mathrm{kcal} / \mathrm{mol}$.

The model complexes $(1-18)$ contain various combinations of halogen donors $(\mathrm{Cl}, \mathrm{Br}$, 
I) and acceptor atoms (O, N, S) (Figure 12). The benchmark interaction energies are given in Table 6. The effect of the chemical environment on the strength of a halogen bond is demonstrated in the series of complexes with oxygen acceptors (formaldehyde and acetone). The weakest interaction is observed in halomethanes, while the same halogen bond in aromatic halides (halobenzenes) is stronger. In trifluorohalomethane complexes, the withdrawal of electrons from the atom involved in the halogen bond results in an increase in the magnitude of the $\sigma$-hole and a reversal of the dipole moment, which make the interaction much stronger. The halogen bonds involving nitrogen in complexes of halobenzenes with trimethylamine are stronger than the respective complexes with oxygen, whereas these with sulfur (e.g., methanethiol) have a stability between those of the oxygen and nitrogen analogues. The iodobenzene...trimethylamine complex is the strongest, with stabilization energy approaching $6 \mathrm{kcal} / \mathrm{mol}$. Heavier halogens (Br and I) interact with the aromatic systems via both dispersion and electrostatic interaction between the $\sigma$-hole and the $\pi$ electrons. These interactions are moderately strong in complexes of bromo- and iodomethane and benzene $(15,16)$. The electrostatic interaction can be strengthened by enlarging the magnitude of the $\sigma$-hole, for example, by fluorination. This is the case for trifluorobromomethane and trifluorochloromethane interacting with benzene (complexes 17 and 18).

The reference dataset is presented to provide accurate values of model halogen bonded complexes. The other, even more important goal is to use this reference to assess the accuracy of selected post-HF methods for investigation of XB complexes. Table 7 lists root-mean-square errors (RMSEs) and mean unsigned errors (MUEs) for the 18 halogenbonded complexes shown in Table 6 and the 22 remaining halogen-containing complexes of the X40 dataset. For the sake of comparison with other types of noncovalent interactions, RMSEs are also listed for our largest dataset (S66) containing 8-point dissociation curves of 66 noncovalent complexes. $\frac{138}{13}$ Note that the RMSE and MUE were calculated with respect to the stabilization energies corresponding to the equilibrium interatomic distances.

Proper treatment of the ESP anisotropy seems to be a key prerequisite for reliable XB 
Table 6: The CCSD(T)/CBS benchmark stabilization energies (in kcal/mol) for halogenbonded complexes. The interacting pair of atoms is provided.

\begin{tabular}{|c|c|c|c|}
\hline No. & complex & XB pair & $\bar{~} \Delta E[\mathrm{kcal} / \mathrm{mol}]$ \\
\hline 1 & chloromethane...formaldehyde & $\mathrm{Cl} \ldots \mathrm{O}$ & 1.17 \\
\hline 2 & bromomethane...formaldehyde & Br...O & 1.72 \\
\hline 3 & iodomethane...formaldehyde & I. . O & 2.38 \\
\hline 4 & trifluorochloromethane...formaldehyde & $\mathrm{Cl} \ldots \mathrm{O}$ & 2.25 \\
\hline 5 & trifluorobromomethane...formaldehyde & Br...O & 3.10 \\
\hline 6 & trifluoroiodomethane...formaldehyde & I. ..O & 4.08 \\
\hline 7 & chlorobenzene. . acetone & $\mathrm{Cl} \ldots \mathrm{O}$ & 1.49 \\
\hline 8 & bromobenzene... acetone & Br...O & 2.43 \\
\hline 9 & iodobenzene. . .acetone & I. ..O & 3.46 \\
\hline 10 & chlorobenzene. . .trimethylamine & $\mathrm{Cl} \ldots \mathrm{O}$ & 2.11 \\
\hline 11 & bromobenzene...trimethylamine & Br...N & 3.78 \\
\hline 12 & iodobenzene...trimethylamine & I...N & 5.81 \\
\hline 13 & bromobenzene...methanethiol & Br...S & 2.32 \\
\hline 14 & iodobenzene....methanethiol & I. . .S & 3.08 \\
\hline 15 & bromomethane...benzene & Br... $\pi$ & 1.81 \\
\hline 16 & iodomethane. . .benzene & I. . $\pi$ & 2.48 \\
\hline 17 & trifluorobromomethane... benzene & Br... $\pi$ & 3.11 \\
\hline 18 & trifluoroiodomethane. . .benzene & I. . $\pi$ & 3.91 \\
\hline
\end{tabular}

calculations. This is, however, not a difficult requirement, and any HF, post-HF, or DFT method should suffice when a larger-than-minimal basis set is used. The semiempirical QM (SQM) methods are the exception among wave-function theories, because they are unable to correctly yield positive $\sigma$-holes on halogens. Consequently, the SQM methods fail in describing halogen-bonded complexes. Note that this is also the case of molecular mechanics, which by definition does not describe $\sigma$-holes.

There exist a number of less computationally intensive techniques that address $\sigma$-hole and yield reasonably accurate results for $\sigma$-hole complexes. In the following paragraphs, we will briefly comment on the most important methods. MP2 has been used for many years with widely varying results that are strongly dependent on the basis set.141 143 With a small basis set, the method may give reasonable results, unfortunately due to unpredictable compensation of errors. When used with large basis sets (or the CBS limit), MP2 typically produces accurate binding energies for hydrogen-bonded complexes and dispersion interactions involving aliphatic species, but overestimates binding energies for dispersion interactions involving

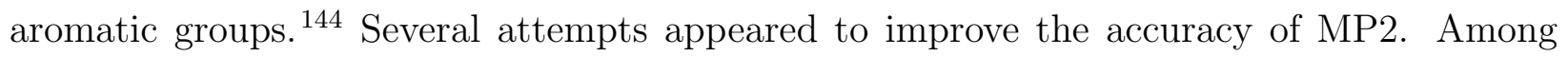


Table 7: : The root-mean-square error (RMSE; in $\mathrm{kcal} / \mathrm{mol}$ and $\%$ of average interaction energy) and mean unsigned error (MUE; in $\mathrm{kcal} / \mathrm{mol}$ ) for the 18 halogen-bonded complexes shown in Table 6 and the 22 remaining halogen-containing complexes of the X40 dataset. The RMSE in the S66 dataset is listed for comparison.

\begin{tabular}{lcccc}
\hline \hline method & RMSE & RMSE (\%) & MUE & S66 RMSE \\
\hline MP2/aug-cc-pVDZ & 0.55 & 15 & 0.43 & 0.79 \\
MP2/CBS & 0.70 & 19 & 0.43 & 0.69 \\
SCS-MP2/CBS & 0.40 & 11 & 0.35 & 0.87 \\
SCS-MI-MP2/CBS & 0.39 & 10 & 0.26 & 0.38 \\
MP3/CBS & 0.45 & 12 & 0.32 & 0.62 \\
MP2.5/CBS & 0.18 & 5 & 0.12 & 0.16 \\
CCSD/CBS & 0.48 & 13 & 0.40 & 0.70 \\
SCS-CCSD/CBS & 0.19 & 5 & 0.12 & 0.25 \\
SCS-MI-CCSD/CBS & 0.06 & 2 & 0.05 & 0.08 \\
BLYP-D3/def2-QZVP & 0.39 & 10 & 0.29 & 0.25 \\
\hline \hline
\end{tabular}

these, the spin component scaled variants (SCS-MP2) $)^{145}$ significantly improved results for noncovalent complexes. The SCS-MI-MP2 method of Distasio et al., $\frac{146}{16}$ which is parameterized specifically for noncovalent interactions, yields binding energies that are significantly more accurate for a variety of interaction types. The SCS technique was also used with a more advanced method covering a higher portion of correlation energy, the coupled clusters with single and double excitations (CCSD). The SCS-CCSD ${ }^{147}$ and SCS-MI-CCSD ${ }^{148}$ methods were parameterized and successfully tested. The latter method in particular provides highly accurate results for diverse noncovalent complexes including halogen bonded ones.

Another type of post-MP2 correction makes use of the fact that, while MP2 tends to overestimate binding energies, MP3 tends to underestimate them. The magnitudes of the over/underestimation predicted by the MP2 and MP3 methods, respectively, are very similar. This is the basis for the MP2.5 ${ }^{149}$ method, which includes an MP2.5 correction, the average of binding energies obtained by MP2 and MP3, $[\Delta \mathrm{E}(\mathrm{MP} 3)-\Delta \mathrm{E}(\mathrm{MP} 2)] / 2$. This method provides very good estimates of the stabilization energies of different types of noncovalent complexes, which closely match the more expensive SCS-MI-CCSD energies. The calculation of MP3 energy, which very roughly corresponds to one iteration in the iterative CCSD calculation, is a critical point. Typically, approximately 20 iterations are needed to converge 
the respective CCSD calculation. Finally, for extended noncovalent complexes, for which wave function calculations are impractical, the DFT method augmented by the dispersion term provides reasonable results.

Of the methods listed in Table 7, the most computationally demanding is the CCSD method, but such demands are not reflected by the accuracy. On the other hand, the SCS-CCSD and particularly the SCS-MI-CCSD method significantly improve upon the pure CCSD. The SCS-MI-CCSD method provides RMSE and MUE very close to the estimated accuracy of the $\operatorname{CCSD}(\mathrm{T}) / \mathrm{CBS}$ benchmark method. MP3 and MP2 methods are lower in computational complexity, but neither performs well. However, the MP2.5 results are much better: RMSE decreases to $0.18 \mathrm{kcal} / \mathrm{mol}$, which is only $5 \%$ of the average interaction energy. Note that the more demanding SCS-CCSD method provides results comparable to those of MP2.5. Better results are only obtained when the parameterized SCS-MI-CCSD/CBS method is adopted. An appreciable feature of the MP2.5/CBS, the average of MP2/CBS and MP3/CBS, is that it is parameter-free. The MP2, following expectations, shows large errors that are reduced when the SCS technique is adopted.

In view of the X40 data set, the DFT augmented with dispersion correction represents a reliable tool for description of noncovalent interactions. This finding, however, has not been reflected by other studies. Among many options, BLYP in a large basis set in combination with D3 correction of Grimme ${ }^{\sqrt{150}}$ was assessed. This method was tested with the S66 dataset and yielded very promising results (see Table 7), but the error for present halogen-bonded and halogenated complexes was somewhat large, although still better than errors obtained with MP3 or CCSD. It must be noted, however, that as we will discuss later, the favorable performance of the BLYP-D3/def2-QZVP method is likely due to significantly supportive compensation of errors.

Among different computational methods tested for halogen-bonded and halogen-containing complexes, the SCS-MI-CCSD and MP2.5 methods exhibit the best performance. The use of the latter method is especially attractive in light of its considerably better computational 
economy; MP2.5 is faster than SCS-MI-CCSD by more than one order of magnitude.

Finally, let us comment on the applicability of all these methods to extended complexes. Recently, we investigated the performance of different WFT and DFT methods to deter-

mine 3-body effects, $\frac{151}{2}$ which becomes topical when moving to extended complexes. Let us stress that 3-body non-additive effects originate in the induction/polarization and dispersion energies; all other terms (such as electrostatic terms) are fully additive. The induction non-additivity is covered in the SCF or DFT energies, while the dispersion non-additivity is covered only when performing higher than 2nd order perturbative calculations. The SCSMI-CCSD and MP2.5 methods exhibited excellent performance; all other methods, including all DFT methods, provided less satisfactory results, indicating that these methods should be used for extended complexes with care.

\subsubsection{XB18 and XB51 Data Sets}

Kozuch and Martin designed two data sets to test the reliability of ab initio non-empirical QM and DFT methods in reproducing XB complex geometries and interaction energies. $\frac{93}{.6}$ The first set, named XB18, contains 18 complexes of halogen diatomics ( $\mathrm{HBr}, \mathrm{HI}, \mathrm{Br}_{2}, \mathrm{I}_{2}$, FBr, FI, ClBr, ClI, BrI) with small electron donors $\left(\mathrm{OCH}_{2}\right.$ and $\left.\mathrm{NCH}\right)$, which are small enough to test geometry optimization. They obtained reference geometries by gradient optimization at CCSD(T)/aug-cc-pVQZ (with the ECPs on Br and I), while the reference interaction energies at the $\operatorname{CSSD}(\mathrm{T}) / \mathrm{CBS}$ limit level were extrapolated from quadruple-zeta and pentuple-zeta basis sets according to equation 5 .

$$
E_{C B S}=E_{L}+\frac{E_{L}-E_{L-1}}{\left(\frac{L}{L-1}\right)^{\alpha}-1},
$$

where $L$ is the cardinal number of the basis set (here $L=5$ ), $\alpha=5$ for singlet coupled pairs and connected triples, and $\alpha=3$ for SCF and CCSD triplet coupled pairs. Note that there is a typo in equation 1 of Ref. $\frac{93}{9}$

In addition to a few ab initio non-empirical QM methods, 42 DFT functionals have been 
examined, covering the whole Jacob's ladder of accuracy. $\frac{152}{152}$ The calculations were performed with the aug-cc-pVQZ basis set. Due to the lack of dynamic correlation, the HF method failed to reproduce XB geometries (characterized by the XB length). Similar to the results with the X40 data set, MP2 tended to notably shorten XB lengths, which could be corrected for by separate scaling of same and opposite spin components in the SCS-MP2 or SCSMI-MP2 methods. Among the DFT functionals, M06-2X, 153 BMK, 154 and CAM-B3LYP $\lcm{177}$ yielded accurate XB lengths.

Kozuch and Martin also compiled a broader set of 51 complexes called XB51 to evaluate the accuracy of interaction energies (Table 8). For some reason, the researchers did not include chlorinated XB donors, and selected only two XB acceptors containing oxygen in the XB51 set. The low number of oxygen acceptors is a drawback, because oxygen is the most abundant XB acceptor in biomolecular XB complexes. On the other hand, they paid special attention to covering a wide range of stabilization energies. The geometries of XB51 complexes were optimized at the $\omega$ B97X/aug-cc-pVTZ level,,${ }^{155}$ while the benchmark stabilization energies were calculated at a composite $\operatorname{CCSD}(\mathrm{T}) / \mathrm{CBS}(\mathrm{MP} 2)$ level (Equation 6).

$$
E_{C B S(M P 2)}=E_{M P 2}(C B S)+E_{C C S D(T)}(a V T Z)-E_{M P 2}(a V T Z),
$$

where the MP2/CBS term was extrapolated from the aug-cc-pVQZ and aug-cc-pVa5Z basis sets according to equation 5 . The benchmark stabilization energies are summarized in Table 8 .

The wave function methods mostly performed in accordance with the results obtained with the X40 data set. HF underestimated the XB stabilization energies by about $4 \mathrm{kcal} / \mathrm{mol}$ on average; MP2 overestimated the stabilization by values between 1.24 and $0.70 \mathrm{kcal} / \mathrm{mol}$ for aVDZ and CBS(Q5), respectively. However, the RMSE for the MP2 method was very close to $1 \mathrm{kcal} / \mathrm{mol}$ regardless of the basis set. Spin-component-scaled variants performed better; the RMSE was 0.44 and $0.33 \mathrm{kcal} / \mathrm{mol}$ for SCS-MP2 and SCS(MI)-MP2, respectively. 
Table 8: The benchmark stabilization energies (in $\mathrm{kcal} / \mathrm{mol}$ ) of the XB51 data set at the CCSD(T)/CBS level. ${ }^{a}$ N-bromosuccinimide, ${ }^{b} \mathrm{~N}$-iodosuccinimide, ${ }^{c}$ pyridine

\begin{tabular}{lcccllcc}
\hline \hline & \multicolumn{3}{c}{ X acceptor } & & \multicolumn{4}{c}{ X donor } \\
\hline X donor & PCH & NCH & $\mathrm{NH}_{3}$ & X acceptor & MeI & $\mathrm{Br}_{2}$ & FI \\
\hline $\mathrm{PhBr}$ & 0.85 & 1.15 & 2.02 & $\mathrm{FCCH}$ & 0.50 & 0.74 & 0.29 \\
$\mathrm{MeI}$ & 0.85 & 1.42 & 2.73 & $\mathrm{PCH}$ & 0.85 & 1.18 & 2.74 \\
$\mathrm{PhI}$ & 0.92 & 1.87 & 3.33 & $\mathrm{NCH}$ & 1.42 & 2.87 & 5.97 \\
$\mathrm{~F}_{3} \mathrm{CI}$ & 0.89 & 3.61 & 5.88 & $\mathrm{FMe}$ & 1.70 & 3.61 & 9.33 \\
$\mathrm{Br}_{2}$ & 1.18 & 3.61 & 7.29 & $\mathrm{OCH}_{2}$ & 2.39 & 4.41 & 9.94 \\
$\mathrm{NBS}^{a}$ & 1.19 & 4.32 & 8.02 & $\mathrm{NH}_{3}$ & 2.73 & 5.95 & 13.36 \\
$\mathrm{FCl}$ & 1.16 & 4.81 & 10.54 & $\mathrm{OPH}_{3}$ & 3.34 & 7.29 & 17.11 \\
$\mathrm{NIS}^{b}$ & 1.53 & 5.91 & 10.99 & $\mathrm{Pyr}^{c}$ & 3.61 & 9.00 & 17.66 \\
$\mathrm{FBr}$ & 2.07 & 7.53 & 15.30 & $\mathrm{HLi}$ & 3.62 & 9.07 & 20.34 \\
$\mathrm{FI}$ & 2.74 & 9.33 & 17.11 & $\mathrm{PdHP}_{2} \mathrm{Cl}$ & 5.05 & 23.11 & 33.79 \\
\hline \hline
\end{tabular}

Extraordinary attention has been paid to DFT. Figure 13 shows the root-mean-square deviations (RMSD) and mean signed errors (MSE) for the stabilization energies. As a clear leader, M06-2X was identified with an RMSE of $0.41 \mathrm{kcal} / \mathrm{mol}$. It was noted that the correct description of charge-transfer was more important than the description of dispersion energy for a reasonable performance of a DFT functional. The amount of exact exchange was critical, as justified by M06-2X, with 54\% HF exchange. It was further shown that the inclusion of a Grimme's-like dispersion correction term ${ }^{[150 \mid 156}$ did not generally improve the performance, no matter what kind of damping scheme was used. This is surprising because the BLYP-D3/def2-QZVP method gave reasonable agreement with reference data from the X40 set. It was pointed out that the delocalization error, which is particularly severe in semilocal functionals, leads to overstabilization of XB when empirical pairwise correction is used. As recently suggested by Otero-de-la-Roza et al., 157 the use of exchange-hole dipole moment dispersion correction ${ }^{158}$ with a functional with low delocalization error may solve the problem. 


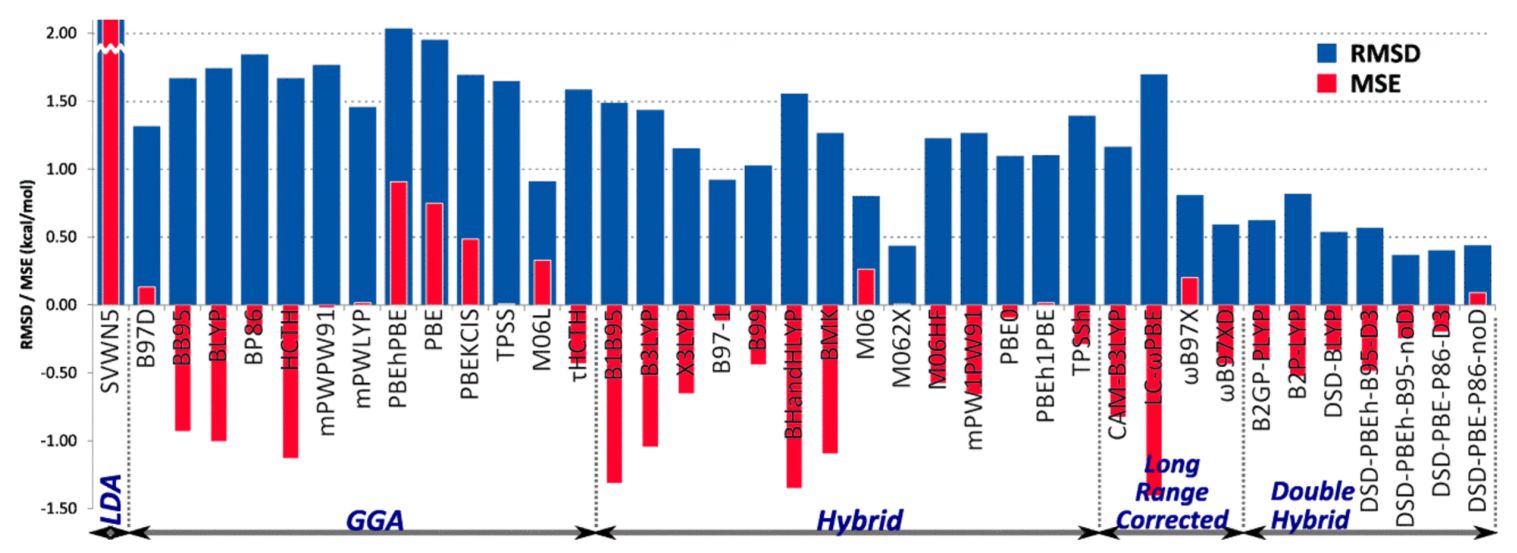

Figure 13: The root-mean-square deviation (RMSD) and mean signed error (MSE) for XB51 stabilization energies calculated with respect to the CCSD(T)/CBS reference. Adapted with permission from Ref. ${ }^{93}$ Copyright 2013 American Chemical Society.

\subsubsection{Benchmarking Other $\sigma$-Hole Interactions}

The two above-mentioned benchmark sets for halogen bonding provide sufficient data for method assessment and development. The situation for other $\sigma$-hole interactions is not as clear-cut. A systematic large-enough CCSD(T)/CBS test set is missing, so the best available data can be perhaps found in the work of Bauzá et al.139 They compiled a set of halogen-, chalcogen-, and pnicogen-bonded complexes and used it to test the quality of ab initio nonempirical QM methods and DFT functionals.

Both the reference geometries and stabilization energies were determined at the $\operatorname{CCSD}(\mathrm{T}) /$ augcc-pVTZ level. The difference from $\operatorname{CCSD}(\mathrm{T}) / \mathrm{CBS}$ is expected to be very low. The reference stabilization energies and equilibrium distances between the pair of closest atoms are summarized in Table 9.159

Figure 14 shows the relationship between the stabilization energies and interatomic distances - the $\sigma$-hole-bonding lengths. There is no general trend reflected by the overall correlation coefficient $\mathrm{R}$ of -0.20 . When, however data from a single electron donor are considered, an inverse correlation (anticorrelation) is apparent. $\mathrm{NH}_{3}$ complexes show very good correlation coefficient $(\mathrm{R}=-0.94)$, which is better than those of both charged series ( $\mathrm{R}$ of -0.72 and -0.86 for $\mathrm{Cl}^{-}$and $\mathrm{Br}^{-}$complexes, respectively). 
Table 9: The reference stabilization energies (in $\mathrm{kcal} / \mathrm{mol}$ ) and equilibrium geometries (in $\AA$ ) of a $\sigma$-hole benchmark set derived at the BSSE-corrected CCSD(T)/aug-cc-pVTZ level.

\begin{tabular}{lllllll}
\hline \hline & \multicolumn{3}{l}{ stabilization energy } & \multicolumn{3}{l}{ equilibrium atom-atom distance } \\
\hline & $\mathrm{Cl}^{-}$ & $\mathrm{Br}^{-}$ & $\mathrm{NH}_{3}$ & $\mathrm{Cl}^{-}$ & $\mathrm{Br}^{-}$ & $\mathrm{NH}_{3}$ \\
\hline $\mathrm{FCl}$ & 28.98 & 26.77 & 9.39 & 2.317 & 2.452 & 2.320 \\
$\mathrm{FBr}$ & 35.48 & 32.73 & 13.39 & 2.440 & 2.588 & 2.355 \\
$\mathrm{SF}_{2}$ & 21.18 & 17.45 & 6.33 & 2.459 & 2.459 & 2.556 \\
$\mathrm{SeF}_{2}$ & 31.07 & 26.68 & 10.65 & 2.490 & 2.678 & 2.453 \\
$\mathrm{~F}_{2} \mathrm{CS}$ & 7.29 & 5.99 & 1.48 & 2.490 & 3.165 & 3.300 \\
$\mathrm{~F}_{2} \mathrm{CSe}$ & 10.73 & 8.90 & 2.38 & 2.899 & 3.113 & 3.230 \\
$\mathrm{~F}_{3} \mathrm{PS}$ & 6.37 & 5.10 & 1.25 & 3.046 & 3.274 & 3.297 \\
$\mathrm{~F}_{3} \mathrm{PSe}$ & 12.14 & 9.93 & 2.46 & 2.870 & 3.098 & 3.169 \\
$\mathrm{~F}_{3} \mathrm{P}$ & 15.09 & 11.68 & 4.11 & 2.665 & 2.973 & 2.883 \\
$\mathrm{~F}_{3} \mathrm{As}$ & 25.76 & 20.86 & 7.57 & 2.575 & 2.824 & 2.674 \\
\hline \hline
\end{tabular}

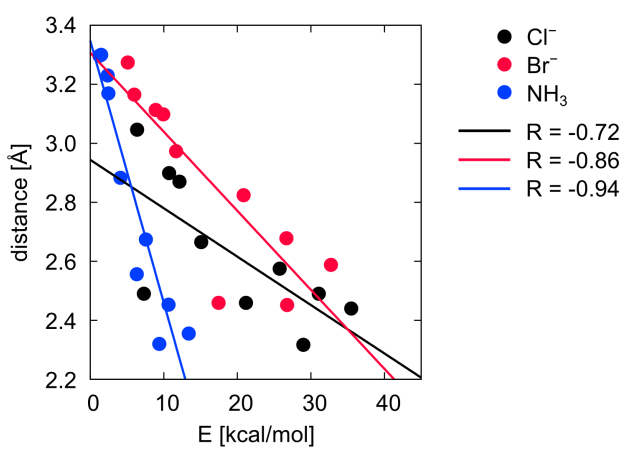

Figure 14: Dependence of the stabilization energy on the interatomic distance (circles). Linear regression fits are shown as lines together with the correlation coefficients $\mathrm{R}$.

Apart from the HF method, all the other methods overestimated the stabilization energy as shown by mean signed error between 0.74 (RI-MP2) and $4.96 \mathrm{kcal} / \mathrm{mol}$ (BP86-D3). Notably, MP2 and RI-MP2 performed rather well (RMSE of 1.18 and $1.23 \mathrm{kcal} / \mathrm{mol}$, respectively). Among the DFT functionals, M06-2X was clearly best, with an RMSE of $1.73 \mathrm{kcal} / \mathrm{mol}$, followed by B3LYP $(\mathrm{RMSE}=2.23 \mathrm{kcal} / \mathrm{mol})$. Similarly to the XB benchmark sets, the dispersion-corrected functionals (BLYP-D3, BP86-D3) performed poorly (RMSE = $5 \mathrm{kcal} / \mathrm{mol}$ ), especially when the complex was charged. The results of BLYP-D3/aug-ccpVTZ are in disagreement with the X40 results. It should be, however, mentioned that the X40 data set contains only neutral complexes, and, furthermore, that a larger basis set (def2-QZVP) was applied in that case. Interestingly, the M06-2X functional was rather weak 
in description of pnicogen bonded complexes. However, the test set compiled by Bauzá et al. is rather imbalanced: it contains 6 halogen-bonded, 18 chalcogen-bonded, and 6 pnicogenbonded complexes, which could distort the final statistical evaluation.

Regarding complex geometries, the authors recommend M06-2X or MP2 at augmented triple-zeta basis set.

\subsection{Semiempirical Methods}

Semiempirical QM methods (SQM) can be applied to more extended molecular systems than QM methods, while still retaining the basic advantage of the QM approach, namely the solution of the Schrödinger equation. Today's successful SQM methods stem from the neglect of diatomic differential overlap approximation (NDDO) of the Hartree-Fock Hamiltonian. 160|161 In addition to the AM1 $1^{162}$ and PM3 $3^{\frac{163}{16}}$ methods, PM6 has become popular, likely thanks to the availability of parameters for a large fraction of the periodic table. 164

The density-functional tight-binding scheme (DFTB), represented by the DFTB2 ${ }^{165}$ and DFTB3 ${ }^{166}$ models, may also be considered a semiempirical quantum chemical method. Unlike NDDO-based methods, DFTB is derived from the Taylor expansion of total energy within the framework of density functional theory. The parameter sets for halogens for the second-order expansion (DFTB2) were developed only recently 167 without much focus on noncovalent interactions. Halogen parameters for DFTB3, the newer variant based on the Taylor expansion into the third order, have been provided as well. 168 The overbinding of halogen bonds in DFTB3 may be corrected for with an empirical correction ${ }^{168}$ that has been parameterized and benchmarked using the set of equilibrium and non-equilibrium interaction energies from the X40 data set. 69

The applicability of SQM methods to noncovalent interactions is generally limited, and the main problem concerns description of dispersion-bound complexes and, perhaps sur-

prisingly, description of hydrogen-bonded complexes.169 SQM methods can be modified to provide a reliable description of these interactions, and the easiest way seems to be to in- 
clude some empirical corrections. In our laboratory, we have developed several versions of such corrections that were parameterized against accurate $a b$ initio interaction energies. The latest version, called $\mathrm{D} 3 \mathrm{H} 4$, provides an accurate description of various types of noncovalent interactions, including hydrogen bonding and dispersion interactions, $\frac{170}{1}$ and has been utilized in a semiempirical-based computer-aided drug design scheme.171

Most of the QM methods describe the $\sigma$-hole satisfactorily, providing that a larger-thanminimal AO basis set is used. The SQM methods use a specially optimized sub-minimal basis set and therefore cannot properly describe the anisotropic density on the halogen atom, which contributes notably to the distinguishing features of the interaction between halogen and electron donor. To our surprise, ${ }^{[172]}$ the standard PM6 method yields interaction energies in various $\mathrm{XB}$ complexes close to the benchmark values. Later, however, we found that the potential energy surface of these complexes can be completely inaccurate, and the reliable characteristics were obtained by accident. The problem is connected to a known failure of SQM methods - underestimated repulsion. Consequently, the nonbonded interatomic distances are too short, which leads to greatly overestimated stabilization energies in noncovalent complexes, particularly in XB complexes. In analogy with corrections to $\mathrm{HB}$ and dispersion interactions, we introduced a correction for XB. We developed an empirical correction parameterized to small complexes for which accurate QM data were available ${ }^{172}$ similar to development of HB correction. Specifically, we used the MP2/augcc-pVDZ dissociation curves for halobenzene... acetone and halobenzene...trimethylamine; this method provides interaction energies for halogen bonded complexes in close agreement with the benchmark $\operatorname{CCSD}(\mathrm{T})$ calculations. The correction was parameterized for chlorine, bromine and iodine. The method was tested on a set of complexes in which substitutions on an aromatic ring modulate the strength of the halogen bond, and it reproduces the reference QM interaction energies with reasonable accuracy. Furthermore, the method was incorporated into a semiempirical scoring function and validated on a series of protein kinase CK2 complexes containing polyhalogenated ligands. .173 
In summary, the latest version of the corrected SQM method, called PM6-D3H4X, provides an accurate description of a wide range of noncovalent interactions, including dispersion, hydrogen bonding, and XB. An important advantage of this technique is that it can be directly applied to other types of $\sigma$-hole bonding. Currently, we are working on extension of the PM6 method to chalcogen- and pnicogen-bonding. In principle, the D3H4X corrections could be combined with other SQM methods such as OM-x, $\frac{174}{R}$ RM1, $\frac{175}{115}$ and SCC-DFTB, 165 but to the best of our knowledge, such enhancements have been pursued only for a DFTB variant. $\frac{168}{1 n}$ our opinion, the use of corrections with PM6 carries a major advantage - the linear scaling algorithm MOZYME, $\stackrel{[176}{ }$ which can be applied to calculations of several thousands of atoms. The PM6-D3H4X version of the original PM6 method is now available in the MOPAC program package. 177

\subsection{Empirical Force Fields}

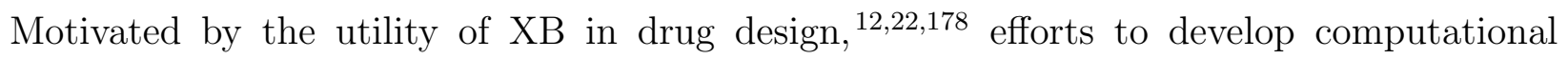
methods even less demanding than SQM have been strengthened. The method of choice in computer-aided drug development and bio-oriented computational research is molecular mechanics (MM) with pairwise effective interaction potentials. In a typical MM approach, one treats the molecules as a set of structureless particles (atoms) connected with harmonic springs, which allows investigation of up to 106 atoms on wide range of time scales. 179

The molecular mechanical noncovalent interactions are explicitly accounted for those atoms that are separated by about three covalent bonds (i.e., the springs), depending on the MM scheme. The use of partial atomic charges representing the electrostatic interactions and Lennard-Jones (LJ) potential to describe van der Waals interactions is rather general

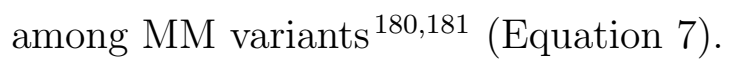

$$
E_{N B}=E_{\text {elec }}+E_{L J}=\frac{1}{4 \pi \varepsilon} \frac{Q_{1} Q_{2}}{r}+\epsilon\left[\left(\frac{\sigma}{r}\right)^{12}-\left(\frac{\sigma}{r}\right)^{6}\right]
$$




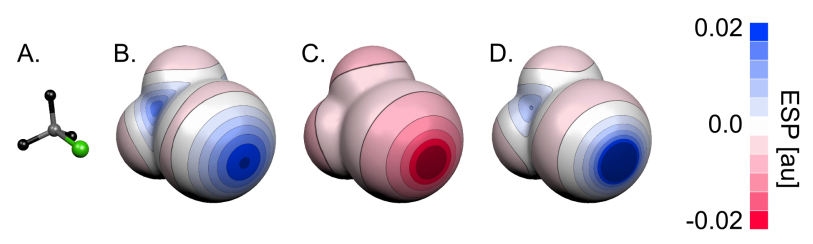

Figure 15: A ball-and-stick model of trifluorobromomethane $\left(\mathrm{CF}_{3} \mathrm{Br}\right)$ is provided $(\mathrm{A})$. A comparison of its molecular electrostatic potential (ESP) derived at QM (B), MM (C), and $\mathrm{MM}+\mathrm{ESH}(\mathrm{D})$ levels is shown and projected on the surface of 0.001 au electron density. Note that the coloring goes from negative reds, through neutral white, to positive blues, and that it is identical for all the three surfaces.

where the nonbonded interaction energy $E_{N B}$ is the sum of the electrostatic $E_{\text {elec }}$ and LJ term $E_{L J}$, and $r$ stands for the interparticle (interatomic) distance, $Q_{1}$ and $Q_{2}$ are the partial atomic charges, $\varepsilon$ is electric permittivity, and $\epsilon$ and $\sigma$ are two interatomic pair parameters of the LJ potential.

Notably, the atomic charges do not correspond to any physical observable, and there is a large ambiguity in their derivation. $182 \underline{184}$ On the other hand, the concept of partial charges is essential for contemporary molecular modeling. Regarding biomolecular simulations and drug design, a few techniques, such as Restricted Electrostatic Potential (RESP) 185 and AM1-BCC, $\underline{186 \mid 187}$ have become widely used in charge derivation. A common drawback of describing electrostatics by atomic partial charges is their inability to produce locally anisotropic electrostatic potentials. From equation 7 , it is also apparent that the LJ potential energy contribution is spherically symmetric.

The ESP anisotropy is important, $\stackrel{188}{ }$ for example, for description of electron lone pairs or, more importantly with respect to this review, for halogen and other $\sigma$-hole bonds. Figure 15 shows MEPs of trifluorobromomethane calculated at the QM level (B) and using atomic partial charges (C). The MEPs indicate that no $\sigma$-hole exists on top of the molecular mechanical model of bromobenzene and that the ESP is progressively negative.

This shortcoming leads to a complete failure of MM calculations on XB complexes, $\frac{173}{173}$ and this fallacy has become paradigmatic in the field of XB. $\frac{189}{}$ Below, we present a few routes to rectification. 

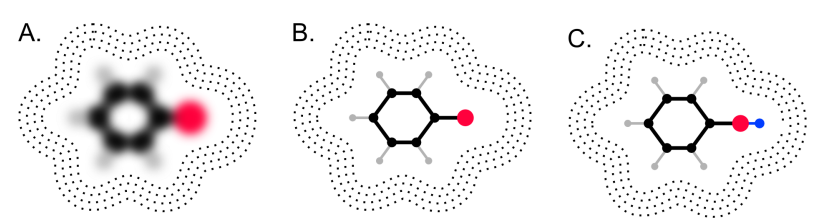

Figure 16: A scheme of the off-center point-charge philosophy shown for bromobenzene. The quantum chemical character is represented by the blurred atoms (A). The atom-centered point charges are calculated to represent the QM-based electrostatic potential (ESP) calculated at a large number of grid points (B). An additional site (in blue) is attached to the bromine (in red) (C) to capture the intrinsic ESP anisotropy while still using the same grid of QM-based ESP.

\subsubsection{Methods with Off-Center Point Charges}

The first attempts to correct for the ESP anisotropy appeared in a number of flavors most likely independently from several research groups within a single year. They collectively used an off-center positive point charge ${ }^{190}$ located near the halogen atom to mimic the $\sigma$ hole. 191194 The extra sites with a negative charge were previously used in description of oxygen and nitrogen electron lone pairs, which are negative, $\frac{195}{19}$ and it seemed natural to utilize their positive counterparts for halogen $\sigma$-holes. The major concerns about these corrections focused on the properties of the extra charge, called the explicit $\sigma$-hole (ESH). The ESP of $\mathrm{CF}_{3} \mathrm{Br}$ derived at the MM level enhanced with an ESH is shown in Figure 15 . Visual inspection reveals that the MM+ESH model is somewhat "harder" than the QM model, with smoother ESP variations. It is, however, better by far than the original MM model, which tends toward an overall negative halogen ESP. The intellectual strategy of the ESH model is depicted in Figure 16. It highlights the fact that one does not need to rely on atomic centers to represent a reference (QM-based) ESP.

From the MM point of view, the ESH particle may carry mass, charge, LJ potential, and other parameters. When the ESH has a non-zero mass, implementation into a MM code is straightforward. In contrast, mass-less particles are more difficult to treat, because the particle mass appears in the denominator in Newton's equations of motion. In these cases, the ESH is represented by a ghost- or dummy-atom, the force on which is redistributed to 
other atoms with non-zero mass in a precise way. $\frac{196197}{A}$ better known example of the use of mass-less dummy atoms is the TIP4P water model.198 Such an approach is available in program packages such as Gromacs $\frac{\sqrt{1992200}}{}$ and Amber. ${ }^{201}$ It must be noted that the mass of the ESH seems to have a negligible effect on the quality of halogen interactions.

The role of the ESH position and charge has been emphasized by numerous researchers. Several charge models differing in the complexity of the input data have been proposed.193 The ESH charge may be fitted by the RESP procedure, which requires a grid of electrostatic potential points. The grid is usually derived from the electron density calculated at a QM level. For the Amber family of force fields, ${ }^{202} \mathrm{HF} / 6-31 \mathrm{G}^{*}$ is recommended. ${ }^{203}$ All of the atoms are fitted at the same time, resulting in an all-fit model. The work of Ibrahim 191 and Rendine et al. ${ }^{[192}$ relied on this scheme. Compared with MM calculations, this QMbased charge derivation represents a bottleneck for high-throughput virtual screenings, and simplification is desired.

If the partial atomic charges of a molecule are known (e.g., from a database), the charge of the halogen without ESH can be split into two contributions - the charge of the ESH and the remainder of the halogen. Typically, a charge of approximately $0.2 \mathrm{e}$ is used for ESH. Jorgensen and Schyman applied this model to enhance the OPLS-AA force field. $\frac{194}{1}$ They used a charge of 0.075 e for chlorine $\sigma$-hole, and 0.10 e for the bromine one. This approach effectively replaces the atomic point charge with a dipole moment, the magnitude of which can be tuned by the ESH-halogen distance and charge splitting. Because no RESP fitting of the charges is performed, the model is referred to as no-fit. In terms of reproducing the QM-based ESP, the all-fit model performs better than the no-fit model. 193

To be complete, yet another scheme has been presented. The ESH charge is taken as an adjustable parameter and the rest of atomic point charges is fitted by RESP. Hence the scheme is referred to as rest-fit. ${ }^{193}$ The quality of the resulting ESP is somewhere between the all-fit and no-fit schemes. The advantage lies in more refined user control of the ESH charge, and consequently its Coulomb interaction, which can be arbitrarily enhanced if needed. 
The position of the ESH can be either fixed or flexible. The fixed ESH requires one additional parameter (the ESH-halogen distance), while the flexible ESH requires a few more parameters, including force constants for stretching the ESH-halogen bond and bending carbon-halogen-ESH angle.

In the all-fit model, the ESH charge and ESH-halogen distance are not independent, and no unique solution exists for their choice. ${ }^{[193}$ We suggested that a distance between 1.2 to 1.5 $\AA$ would be reasonable. A recent study proposed $1.33 \AA, 204$ which agrees with the previous findings. Slightly higher equilibrium distances were used in OPLS-AA, i.e., $1.6 \AA$ for the ESH of chlorine and bromine and $1.8 \AA$ for the ESH of iodine. Even higher distances were derived by Ibrahim, who proposed that the ESH-halogen distance is equal to the van der Waals radius of the respective halogen $\frac{191}{}$ (about $2.1 \AA$ for bromine). This leads to an abnormal situation in which a typical halogen bond between a bromine and a carbonyl oxygen with XB length of about $3 \AA$ is modelled by a ghost atom, which is less than $1 \AA$ from the carbonyl oxygen and deeply penetrates into its van der Waals sphere.

Some variants add a repulsive wall onto the ESH via LJ parameters. $\frac{191}{1}$ The higher the ESH-halogen distance, the more such repulsion is needed for the stability of computer simulations. Its role in the XB quality has not yet been systematically studied.

The off-center point charge models improve the structure and dynamics of protein-ligand complexes. On the other hand, there is still much room for improvement in terms of thermodynamics and liquid properties. For instance, the OPLS-AAx study by Jorgensen and Schyman ${ }^{194}$ reported minor or no improvement in the density of liquid monohalogenated benzenes when compared to the uncorrected force field and experimental results.

\subsubsection{Electric Multipole Expansion}

Another class of molecular mechanics of XB goes beyond the partial charge approximation and uses electric multipole expansion. The halogen ESP anisotropy can be described by a sum of multipoles, namely by a point charge (monopole), dipole, quadrupole, etc. An 
isolated halogen atom has its electric multipoles averaged to zero. However, a permanent quadrupole moment is an intrinsic property of isolated halogens with the valence electron configuration $\mathrm{p}_{x}^{2} \mathrm{p}_{y}^{2} \mathrm{p}_{z}^{1}$. It is thus common to truncate the multipole expansion after the quadrupoles. ${ }^{205206}$ It was shown, however, that the point electric quadrupole sufficiently represents the electrostatic situation on a covalently bonded halogen ${ }^{207}$ and that excluding the dipole from the expansion increases the certainty of the quadrupole by about one order of magnitude.204

Torii and Yoshida ${ }^{205}$ fitted the atomic point charges of an entire molecule with a quadrupole moment located only on the halogen. They found that the $\mathrm{zz}$ component of a traceless quadrupole (where the $\mathrm{z}$-axis coincides with the $\mathrm{R}-\mathrm{X}$ covalent bond) is insensitive to aromatic substituents, and the substitution effect is rather manifested by the variations of the atomic point charges. Titov et al. ${ }^{204}$ compared the performance of off-center charge model and the multipole expansion in reproducing QM-based molecular electrostatic potential. Their major finding was that both models performed equally well when the non-halogen part of the molecule was described in the same way. As opposed to off-center charges, the use of multipole expansion in molecular dynamics software is limited.

\subsubsection{Aspherical Interatomic Potentials}

Shing Ho's research group adopted a completely different strategy to tackle XB. Rather than searching for optimal parameters for current MM functional forms, they derived a new potential energy function to cover the anisotropy of the XB interaction. ${ }^{208}$ Both the LJ and the electrostatic terms were modified. Altogether, seven parameters per halogen are needed, which creates a model that is more complicated than the point-charge-based and multipole-based models. Parameterization of bromine was performed employing correlated QM calculations and validated on experimental studies of four-way DNA junctions with bromouracil. ${ }^{208}$ Recently, the parameter set was completed with the addition of chlorine and iodine. ${ }^{209}$ Some of the parameters are coupled, which agrees with findings from studies of 
extra-charge models. $\frac{193}{193}$ However, the potential energy function has been implemented only into the Amber 201 program package so far, which is somewhat limiting.

\subsection{Methods for Virtual Screening}

At early stages of drug discovery, the importance of computational models has been increasing rapidly. A common task of computer-aided drug design is to rank drug candidates according to their propensity to become a drug. ${ }^{210 \mid 211}$ Such virtual screenings typically work with huge databases of compounds, and the use of fast-scoring methods is imperative. The scoring functions are also employed in determination of an unknown target-ligand binding pose, which is a prerequisite for many computational studies. A number of possible ligand orientations in the binding site is generated by an algorithm, 212,214 and the poses are subsequently scored according to their presumed affinity. $215 \mid 216$

Regarding XB, several methods have been developed that are even more approximate (and thus faster) than those used in molecular dynamics simulations. An empirical type of scoring function that recognizes the halogen bonding pattern on the basis of distance and angular parameters has been developed in F. Hoffmann-La Roche AG. ${ }^{217}$ The covalent and noncovalent interactions are converted into a network that is used for scoring the targetligand binding. This scoring function, named ScorpionScore, is a combination of pairwise and many-body terms. Interestingly, similar importance was assigned to halogen bonding and cation. . $\pi$ interactions.

Three variants of a knowledge-based scoring function (XBPMF) have been developed by Liu et al. ${ }^{218}$ XBPMF was trained on experimental X-ray geometries found in the Protein Data Bank. Such conversion of structural information into energy dependence represents a general strategy in scoring function design, $\underline{219220}$ and it became clear that one-dimensional (distancedependent) potential might not be sufficiently accurate for XB. Liu et al. extended their previous work on two-dimensional potentials of hydrogen bonding 221 to XB. They performed tests on a set of 162 protein-ligand complexes with known binding affinities (PDBbind, 


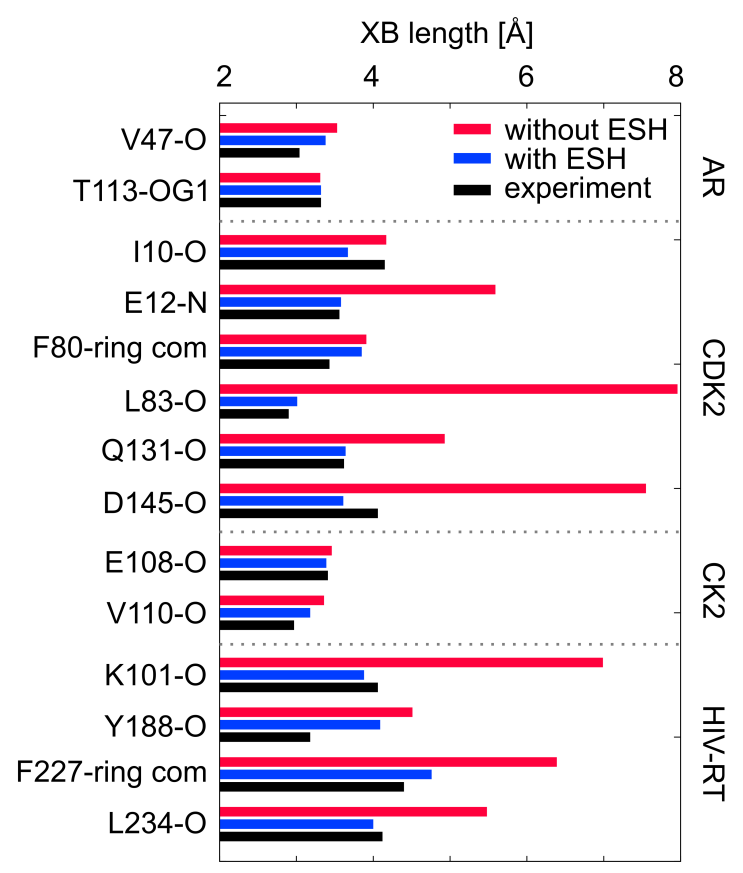

Figure 17: Length of halogen-oxygen contacts with various amino acids of four proteins (AR, aldose reductase; CDK2, cyclin-dependent kinase 2; CK2, protein kinase CK2; HIVRT, human immunodeficiency virus reverse transcriptase) averaged over all of the ligands. The results without the ESH model (red) and with the ESH model (blue) are compared with the experimental X-ray crystallography (black). com stands for center of mass.

ver. 2012 $\frac{222}{2}$. When compared to several popular scoring functions, XBPMF performed moderately well. It was rather weak in selecting the best binding pose, but strong in ranking ligands binding to a particular protein.

Of course, if a docking/scoring technique employs a molecular mechanical force field, one can enhance the performance by the off-center charges (see Section 3.3.1). This was done 223 for the first time for the DOCK6 program package, $\stackrel{214}{2}$ using the no-fit scheme as described by Kolář et al. ${ }^{193}$ On a set of about 100 experimental protein-ligand geometries, it was shown that the number and quality of halogen-oxygen contacts are improved. Figure 17 shows the average length of halogen-oxygen contacts between four proteins studied and their ligands. Docking with the ESH model performed better than docking without, as compared with the X-ray experimentally derived geometries.

F. Böckler's research group developed another physics-based scoring function. Using 
second-order Møller-Plesset perturbation theory (MP2), they designed a simplified model of halogen interaction with a protein-backbone carbonyl oxygen. ${ }^{224}$ The so-called XBScore is an extension of their previous systematic study of protein backbone interactions. ${ }^{225}$ A polynomial dependence of the halogen bond geometry (XB length and angle) is introduced in XBScore. Training of the scoring function was performed on halobenzene...N-methylacetamide complexes, and validation was performed on the full Protein Data Bank. XBScore was particularly successful in re-identification of existing protein-ligand halogen bonds. ${ }^{224}$

Although not fully documented, recent versions of some commercial docking/scoring packages like Glide ${ }^{\sqrt{226}}$ and Gold $\sqrt{2271228}$ offer a limited description of halogen bonds. As admitted in the manual pages, the XB is expected to have only a minor effect on the results, unless a strong XB is established.

\subsection{Semiempirical QM Scoring Functions}

It should be mentioned here that the binding affinity of a ligand to a protein represents a delicate balance between the free energy gained upon ligand binding and free energy needed to desolvate both the ligand and the protein's active site. Furthermore, entropy should be considered. The problem is that free energy components in a composite scoring function are larger than the experimental absolute binding free energy, and the difference between a tight-binding inhibitor (with nanomolar activity) and the weakest detectable inhibitor is only about $5 \mathrm{kcal} / \mathrm{mol}$. Therefore, a reliable scoring function must consist of different energy terms describing the particular interaction as accurately as possible.

We have focused on the binding free energy between protein and ligand. Because it is necessary to consider the whole protein having several thousands of atoms, the use of QM methods is limited, and MM methods do not provide results that are accurate enough in all situations. SQM methods present a compromise between accuracy and computing economy. For a long time, the use of SQM energies was limited by the computing power. However, nowadays is has become more accessible to run these kind of calculations for thousands of 
molecules, ${ }^{229}$ which makes this approach competitive with more established virtual screening techniques.

The use of SQM methods in drug design was pioneered by Merz's group 2301231 who used AM1 and later PM3 methods. Because neither of these methods describe halogen bonding, the use of the respective scoring function for ligands containing halogens is limited. In our laboratory, we developed a novel scoring function based on the corrected PM6 SQM method. 171 The most recent modification of the method, PM6-D3H4X, describes halogen bonding between ligand and protein (as well as other types of noncovalent interactions) well, and the method is thus suitable for assessing interactions between proteins and halogenated ligands.

\section{Computational Studies}

\subsection{Directionality of $\sigma$-Hole Interactions}

The ability of halogen bonds to adopt certain geometric patterns with a higher likeliness than others is one of their most characteristic properties. Certainly, the broad applications of XB in biosciences and material sciences are partially thanks to it. ${ }^{7}$ Experimentally determined structures of $\mathrm{XB}$ complexes show that the $\mathrm{R}-\mathrm{X}$. . Y angle in the halogen bond, where $\mathrm{X}$ is a halogen atom and $\mathrm{Y}$ is an electron donor, tends to be close to $180^{\circ}$, i.e., a linear arrangement.

Two surveys ${ }^{2321233}$ of the Cambridge Structural Database ${ }^{234}$ and one ${ }^{235}$ of the Protein Data $\operatorname{Bank}^{236}$ suggested that the tendency to linearity is stronger for heavier halogens than lighter ones. However, due to the lower relative crystallographic occurrence of XBs involving iodine, the data for iodine are prone to larger statistical uncertainties than those of chlorine and bromine, and thus there is some difficulty in obtaining quantitative trends.

We have investigated the directionality of halogen bonding in a series of stand-alone halogenated aromatics and halogen benzene complexes with argon and hydrogen fluoride. Figure 18 shows the angular dependences of the stabilization energy of chloro-, bromo-, and 
iodobenzene with hydrogen fluoride calculated at the B3LYP-D3/def2-QZVP level with the effective core potentials on iodine $\frac{65}{65}$ These were obtained by varying the $\mathrm{C}-\mathrm{X}$...F angle between $90^{\circ}$ and $270^{\circ}$, where $180^{\circ}$ corresponds to a linear XB. The X...F-H angle was kept $180^{\circ}$.
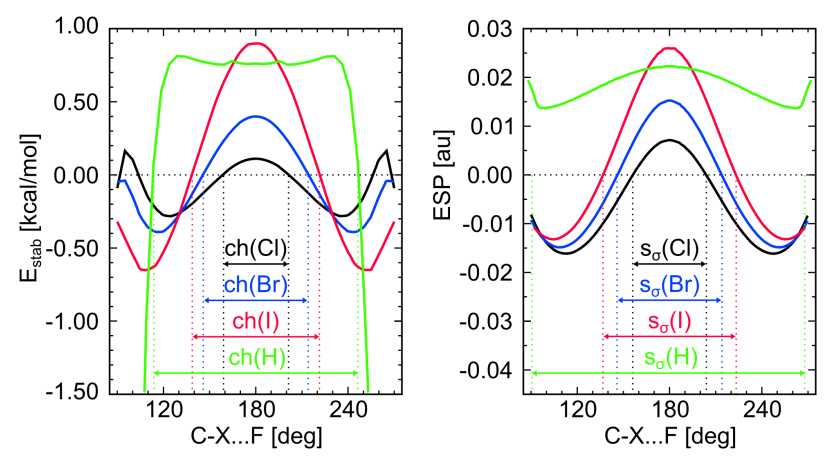

Figure 18: Left panel: The stabilization energy profiles of halobenzene complexes with hydrogen fluoride calculated at the B3LYP-D3/def2-QZVP level. The angular channel, where the interaction is attractive, is labeled $\operatorname{ch}(X)$, where $\mathrm{X}$ stands for the halogen. Right panel: The electrostatic potential (ESP) angular profiles calculated at 0.001 au electron density surface using the same computational method. The angular variants of the sizes of $\sigma$-hole are labeled $s_{\sigma}(X)$, where $\mathrm{X}$ stands for the halogen.

The stabilization energy profiles depend on the atomic number of the halogen donor. The interaction with the hydrogen fluoride is attractive in a linear conformation but repulsive in a perpendicular direction with respect to the $\mathrm{C}-\mathrm{X}$ covalent bond. Such behavior resembles the ESP surface of halogens. As a consequence, by varying the $\mathrm{C}-\mathrm{X}$...F angle, the complex maintains attractive interaction. The heavier the halogen involved in the $\mathrm{XB}$, the more variability is allowed to maintain the stability (i.e., positive stabilization energy). Moreover, the size of $\sigma$-hole $\left(s_{\sigma}\right)$ reflects such changes (Figure 18 , right). In the SAPT sense, the angular variations of the halogen bond are caused not only by the electrostatic energy, but also by the exchange-repulsion energy; the angular variation of the dispersion energy is considerably lower. This is consistent with another approach of dissecting dispersion interaction described by El Kerdawy et al. ${ }^{109}$ Based on two-dimensional scans at the frozen-core $\operatorname{CCSD}(\mathrm{T}) /$ augcc-pVQZ level using argon atom as a probe, they found that the sum of dispersion and repulsion is significantly angular dependent. 
In our previous work, we stated ${ }^{65}$ that “...the channel allowing the approach of HF to halogenbenzenes (with the attractive interaction energy) is the narrowest for $\mathrm{Cl}$ and the broadest for I. Alternatively these values indicate that the directionality of halogen bonds is largest for chlorobenzene and becomes the smallest for iodobenzene," and by "the directionality," we meant the channel of attractive interaction energy. We admit that our use of the terminology was not particularly fitting, and we investigated the problem further ${ }^{[66}$ We have shown that one must distinguish between such directionality (in our work, represented by the channel) and the tendency to linearity. The above-mentioned experiments suggest, indeed, that the tendency to linearity should increase with increasing atomic number of the halogen.

The energetic penalty brought upon an angular distortion increases with increasing size of the $\sigma$-hole. As shown in Figure 18, the ESP profile is steepest in case of iodine, hence "the force acting on the electron donor is largest when iodine is involved, favoring the linear arrangement of the XB more than in the cases of bromine and chlorine. "[6] In other words, the gradient of the ESP is higher for heavier halogens, which consequently determines the linear geometry of the XB. Tsuzuki et al. drew similar conclusions ${ }^{237}$ based on computational investigation of complexes of halogenbenzenes with pyridine. Furthermore, they found that the magnitude of angular dependence is dependent on the XB strength. For weaker XBs, the angular variations of stabilization energy were weaker than for stronger XBs. The strength of XBs was modulated by changing the XB length. ${ }^{237}$

It is intriguing to see the effect that an aromatic substitution has on the angular dependence of ESP. Figure 19 shows the relative ESP angular profiles calculated on the surface of electron density of 0.001 au for all benzene derivatives as previously described $\frac{\sqrt[65]{6}}{\text { (i.e., with }}$ $-\mathrm{CH}_{3},-\mathrm{F},-\mathrm{NO}_{2}$ or $-\mathrm{CN}$ groups in the meta- and/or para- positions with respect to the halogen). For sake of comparison, the profiles were normalized so that the magnitude of the $\sigma$-hole was equal to 0 au (i.e., the ESP for angle $180^{\circ}$ ). The curves naturally cluster with respect to the halogen involved in a possible XB. While the exchange of the halogen has a rather strong effect on the ESP profile and its steepness, aromatic substitutions, on the 


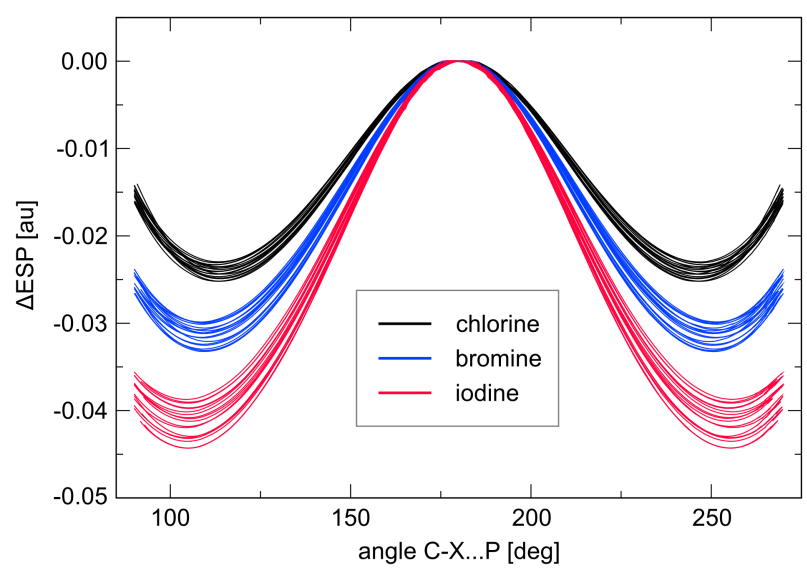

Figure 19: An aggregation of the electrostatic potential (ESP) profiles from our previous work ${ }^{65}$ normalized with respect to a $180^{\circ}$ angle. The ESP profiles were derived on the surface of electron density of 0.001 au. Chlorobenzene (black), bromobenzene (blue), and iodobenzene (red) derivatives are shown.

other hand, vary the profiles considerably less. Consequently, the tendency of a halogenated aromatic to adopt a linear XB geometry is affected much more by the nature of the halogen involved in the XB than by the chemical substitutions on the aromatic ring, which is rather surprising.

Protein backbone carbonyl oxygens are electron donors with high natural abundance. Thus, an exploration of their propensity for halogen bonding is desirable. Three-dimensional spherical scans were performed to characterize the directionality in sub-optimal XB arrangements. $\frac{225}{}$ N-methylacetamide was chosen to model the protein backbone, and halogenbenzenes as the ligand. Unrelaxed scans were carried out at the MP2/TZVPP level using constrained monomer geometries. It was confirmed that, indeed, the interaction energy is angular dependent and decreases notably upon even slight XB deformation. A decrease of about $50-60 \%$ was reported for angular deviations of about $30^{\circ}$, but it must be kept in mind that secondary interactions may contribute to the stabilization, especially for large distortions. Relaxation upon distortion would increase the complex stability. These results were later utilized in design of a scoring function of halogen bonds. 224

Analogously to XB, other $\sigma$-hole interactions seem to be driven by ESP anisotropy. $\frac{238}{2}$ 
Work by Adhikari and Scheiner ${ }^{239}$ concluded that the propensity to linear arrangement is much higher for halogen, chalcogen, and pnicogen bonds than for hydrogen bonds. Based on SAPT decomposition, it was further revealed that, similarly to XB, the angular sensitivity of other $\sigma$-hole interactions is strongly determined by the exchange-repulsion energy component. 239

\subsection{Hydrogen vs. Halogen Bonding}

Hydrogen and halogen bonds exhibit numerous similarities but also some important differences that can be important in bio- and material sciences. As such, they are often the subject of direct comparisons. One of the most important differences is their directionality. At the end of the last century, i.e., before the concept of halogen bonding had been widely spread, directionality of hydrogen bonds was considered an important property of this noncovalent interaction. 240,242

Based on the S66 $\frac{138}{13}$ and $\mathrm{X} 40^{69}$ benchmark data sets, the strength of the two interactions is comparable. The total stabilization energies of $\mathrm{HB}$ and $\mathrm{XB}$ are generally similar, but this is not true for their decomposition (e.g., by the SAPT scheme). This represents another very important difference between the two interactions. The electrostatic energy is, no doubt, the most attractive contribution to $\mathrm{HB}$, while dispersion and induction energies are less important (although they cannot be neglected). The situation is different for XB, and its characteristic properties are due to the concerted action of dispersion, electrostatic, and induction/charge-transfer energies.

HB has been considered a $\sigma$-hole interaction by some researchers. ${ }^{7|18| 91 \mid 92}$ However, the nature of $\sigma$-hole as a region of positive ESP on hydrogen differs from that on halogens. As detailed by Clark, 18 the hydrogen $\sigma$-hole is caused by a shift of electron density along the $\mathrm{R}-\mathrm{H}$ covalent bond. As a consequence, the hydrogen nucleus is exposed because there are no other electrons to shield it (the only one participates in the covalent bond). Another difference mentioned by Clark ${ }^{[18}$ is that, in terms of ESP, the hydrogen is generally positive 


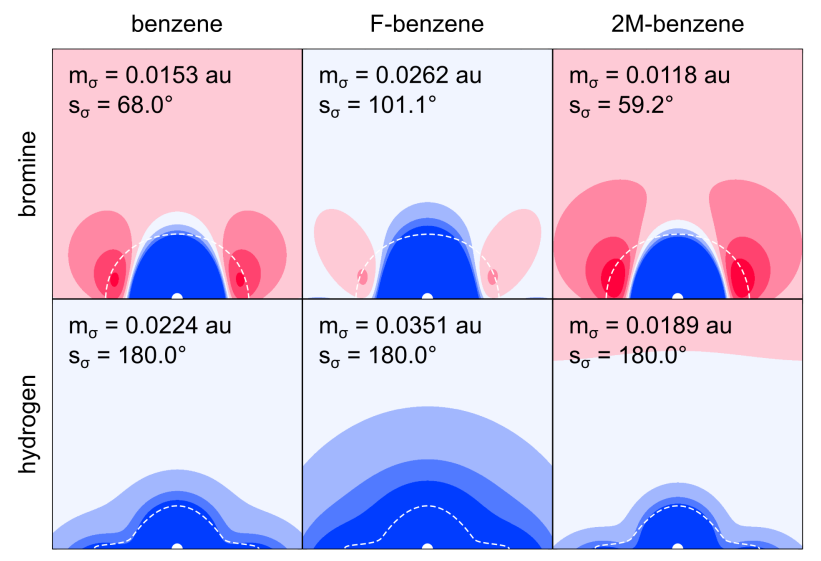

Figure 20: Two-dimensional electrostatic potential maps of substituted bromobenzenes (upper panels) and benzenes (lower panels) calculated in the plane of the aromatic ring. Positive values are in blue and negative in red. All-hydrogen, para-fluoro-, and meta-dimethyl- analogues are shown in columns labeled benzene, F-benzene, and 2M-benzene, respectively. The magnitudes $\left(\mathrm{m}_{\sigma}\right)$ and sizes $\left(\mathrm{s}_{\sigma}\right)$ of the $\sigma$-holes are provided. The dashed white line stands for the surface of 0.001 au electron density. The white hemicircle is either bromine (upper

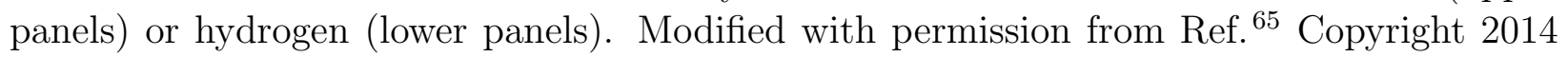
Royal Society of Chemistry.

everywhere.

Wolters et al. $\frac{125}{1}$ highlighted some differences between $\mathrm{HB}$ and $\mathrm{XB}$ from the molecular orbital perspective. Based on relativistic DFT, they found that there is a significantly stronger HOMO-LUMO interaction in XB. They also noted that the heteronuclear dihalogens possibly involved in XB are less polar that hydrogen halides available for HB.

The literature on the directionality of hydrogen and halogen bonds is quite rich. $\frac{3|7 / 54 / 65| 78 \mid 91 / 237 / 243}{1}$ As stated by Shields et al., 91 the different sensitivity of the interaction strength of halogen and hydrogen bonds on the angular deviations can be attributed to the presence or absence of lone electron pairs in the valence shell of the halogen or hydrogen. Figure 18 shows the stabilization energy profiles of halobenzene and benzene complexes with hydrogen fluoride. The comparison arises from B3LYP-D3/def2-QZVP calculations, as previously described in detail. $\underline{65}$ The hydrogen analogue profile is notably shallower than the halogen one, which points to the low sensitivity of benzene complexes with hydrogen fluoride on angular deviations, which is in full agreement with the results for benzene analogues and pyridine. 237 
Furthermore, it is evident that the $\sigma$-hole on halogens is typically a well-bounded positive region on the isodensity surface, while the ESP on hydrogens is positive everywhere (Figure 18. right). This is also shown in two dimensions in Figure 20. It is worth noting that, upon substitution of the aromatic ring, the hydrogen ESP changes differently than the halogen ESP. For example, when adding fluorine to the para-position relative to bromine on bromobenzene, the $\sigma$-hole magnitude increases from 0.015 to $0.026 \mathrm{au}$. Correspondingly, the $\sigma$-hole magnitude on the benzene analogue changes from 0.022 to $0.035 \mathrm{au}$. The change of the $\sigma$-hole size is, however, completely different: the halogen $\sigma$-hole size increases, while it is virtually unchanged in the case of the hydrogen analogue (Figure 20).

Finally, a very important difference between hydrogen and halogen bonds concerns the solvation energy. HB is mostly more polar than XB, and consequently, the solvation/desolvation energy of $\mathrm{HB}$ complexes is higher than that of $\mathrm{XB}$ complexes. This feature represents an important advantage of XB, particularly in protein-ligand association.

\subsection{Vibrational Spectra of Halogen-Bonded Complexes}

Formation of an $\mathrm{R}-\mathrm{X}$...Y halogen bonded complex is accompanied by changes in bond lengths and vibration frequencies of interacting subsystems. Larger changes are expected for the halogen donor R-X. For R-H. .Y hydrogen bonding, which is similar to halogen bonding, it was believed for a long time that formation of $\mathrm{HB}$ is accompanied by lengthening of the $\mathrm{R}-\mathrm{H}$ bond and lowering of the $\mathrm{R}-\mathrm{H}$ vibration frequency (so-called red shift). These characteristics were considered "fingerprints" by which the formation of HB could be recognized. At the end of the last century, we showed $\frac{244}{2}$ that HB formation can be accompanied not only by red shift of $\mathrm{R}-\mathrm{H}$ vibration frequency and elongation of the $\mathrm{R}-\mathrm{H}$ bond, but also by blue shift of this vibration and contraction of the bond. This finding initialized the introduction of a new definition of HB. 245

The first study on vibrational shifts in R-X...Y halogen-bonded complexes suggested 246

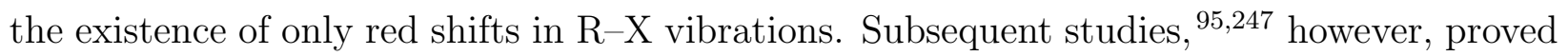


the existence of blue shifts in some halogen bonded complexes. The origin of blue and red shifts was investigated ${ }^{248}$ in detail for 12 halogen bonded complexes in which the electron donor was either $\mathrm{HCN}$ or $\mathrm{NH}_{3}$ and the halogen donors were XF, $\mathrm{XCN}, \mathrm{XNO}_{2}$, and $\mathrm{XCF}_{3}(\mathrm{X}=\mathrm{Cl}, \mathrm{Br})$. The stabilization energies of all these complexes were between 1 and $6.9 \mathrm{kcal} / \mathrm{mol}$ (at frozen-core MP2/6-311++G(3df,2p) level), and all X...Y distances were shorter than the sum of the van der Waals radii of the respective atoms. Six of the complexes exhibited blue shifts $\left(3-17 \mathrm{~cm}^{-1}\right)$, which were always accompanied by a decrease in $\mathrm{R}-\mathrm{X}$ bond length. All of the $\mathrm{NH}_{3}$ complexes displayed red shifts, even with those electron donors that exhibited a blue shift with HCN. Different shifts depend on the properties of the halogen donor and the electron donor. A necessary but not sufficient condition for blue shifting is that the derivative of the $\sigma$-hole donor's permanent dipole moment is opposite to the direction of the electric field induced by the acceptor..$^{249} 250$ If this condition is satisfied, a further requirement concerning the nature of the electron donor (contribution of the induced dipole moment derivatives to the vibration shift should not be larger than that of the permanent dipole) should be fulfilled.

Our results on XB were fully consistent with these conditions developed earlier for blueand red-shifting hydrogen bonds. The blue shifts of $\mathrm{R}-\mathrm{X}$ stretching frequencies were also found for six complexes of anesthetics (chloroform, halothane, enflurane, and isoflurane) with formaldehyde. $\frac{251}{25}$ The binding energies of these complexes calculated at the CCSD(T)/CBS level were between 2.83 and $4.21 \mathrm{kcal} / \mathrm{mol}$. In all complexes, the $\mathrm{C}-\mathrm{X}$ bond length (where $\mathrm{X}=\mathrm{Cl}, \mathrm{Br}$ ) was slightly shortened in comparison to the isolated $\mathrm{XB}$ donor, and an increase in the $\mathrm{C}-\mathrm{X}$ stretching frequency was observed. The electrostatic interaction was excluded as being responsible for the $\mathrm{C}-\mathrm{X}$ bond contraction. On the other hand, it was suggested that contraction of the $\mathrm{C}-\mathrm{X}$ bond length could be explained in terms of the Pauli repulsion (the exchange overlap) between the electron pairs of the oxygen and halogen atoms in the complexes.

In conclusion, structural and vibration-frequency changes upon formation of a halogen 
bond are similar to those in hydrogen bonded complexes. The $\mathrm{R}-\mathrm{X}$ bond length can increase or decrease upon formation of a halogen bond, and the respective vibration frequency can be shifted to lower (red shift) or higher (blue shift) energies. The changes in the bond length and vibration frequency are considerably lower for halogen bonded complexes than for hydrogen-bonded complexes, which is due to the different nature of the bonding (the presence of light hydrogen in $\mathrm{HB}$ ).

\subsection{Structural Studies of Small Molecules}

The utility of computational tools in understanding crystal packing and noncovalent-driven self-assembly is notably wide. Quantum chemistry is well-suited to study interactions of molecules with low spatial flexibility, and the lack of solvation dynamics plays a favorable role.

Early surveys of the Cambridge Structural Database (CSD) ${ }^{234}$ focused on halogen contacts were augmented by quantum chemical analyses. $\frac{1231232}{1.20}$ Ouvrard et al. inspected the interactions of dihalogens. ${ }^{252]} \mathrm{A}$ comparative study of halogen and other $\sigma$-hole bonds by Bauzá et al. ${ }^{[233}$ combined the CSD survey and quantum chemistry. Based on BP86-D3/def2TZVP calculations, Bauzá et al. showed that for $\sigma$-hole interactions with a nitrogen as the electron donor, halogens are energetically more favorable than chalcogens or pnicogens as electron acceptors. In contrast, when the electron donor is an aromatic ring, pnicogens are preferred over halogens and chalcogens. ${ }^{233}$

Iwaoka et al. used a combination of NMR spectroscopy, NBO analysis, and the QTAIM approach to study Se... O intramolecular contacts. $\frac{253}{25}$ Because of a bond critical point (BCP) identified between Se and O, the researchers concluded that the interaction is of covalent rather than electrostatic character, which is, however, limited to the QTAIM diction. It must be noted that in other schemes, the interaction might be classified differently.

We studied crystals of hexahalogenbenzenes $\left(\mathrm{C}_{6} \mathrm{~F}_{6}, \mathrm{C}_{6} \mathrm{Cl}_{6}\right.$, and $\left.\mathrm{C}_{6} \mathrm{Br}_{6}\right)$ and benzene $\left(\mathrm{C}_{6} \mathrm{H}_{6}\right)$ using DFT and DFT-SAPT ${ }^{254}$ Halogenbenzene crystals are rich in halogen-halogen 
contacts, some of which could be classified as a dihalogen bond. Such interaction is characterized by contact of the positive $\sigma$-hole of one halogen with the negative belt of a second halogen. To our surprise, we found that the dihalogen bond does not contribute significantly to the stability of the crystals. The dispersion energy, both in the SAPT sense and Grimme's empirical one, seems to be the leading source of stabilization. The differences in the sublimation energy of the crystals can be explained by the dispersion interactions.

Substantial pairwise stabilization energies were found for crystal structures of diiodine complexes with diazabicyclo[2.2.2] octane (DABCO) and 1,3-dithiole-2-thione-4-carboxylic acid (DTCA). ${ }^{94}$ Furthermore, similar complexes with other molecules $\left(\mathrm{Cl}_{2}, \mathrm{Br}_{2}, \mathrm{IF}, \mathrm{ICH}_{3}\right.$, $\mathrm{N}_{2}$ ) were examined using the CCSD $(\mathrm{T}) / \mathrm{CBS}$ and DFT-SAPT methods. Surprisingly, the stabilization energy correlated with the classical concept of electric quadrupole moments of the electron acceptors. Comparing the quadrupoles of $\mathrm{X}_{2}$ molecules, we found that they have different signs for dihalogens and nitrogen. The quadrupoles of the halogens can be schematically written as +--+ , and those of nitrogen as -++- . Evidently, the notation reflects the concept of the $\sigma$-hole. Furthermore, there exists a close correlation between $V_{\max }$ and the quadrupole value. Finally, in contrast to findings from many other DFT-SAPT studies of XB complexes, the induction energy of the complexes of diatomics was the leading SAPT term.

Chalcogen and pnicogen bonding in heteroborane crystals has been studied computationally. $\frac{113}{13} \sigma$-hole analyses and $\operatorname{CCSD}(\mathrm{T}) / \mathrm{CBS}$ calculations were complemented by DFT-SAPT energy decompositions. In our opinion, this is a reliable way of studying noncovalent interactions without making compromises, and the final picture seem to be quite complete. Heteroboranes are prone to halogen bonding in a comparable way as other, more typical halogen donors. Positive $\sigma$-holes were identified on halogens incorporated as exo-substituents, as well as on chalcogens and pnicogens incorporated directly into the borane cage. Further experiments and calculations justified that these $\sigma$-hole bonds are essential for the crystal packing of heteroboranes. $\frac{63}{63}$ 


\subsection{Halogen Bonds in Biomolecules and Medicinal Chemistry Ap- plications}

Halogen bonds have been proven to exist in numerous biomolecular complexes, ${ }^{2351255}$ and their specificity was recognized quite early ${ }^{256}$ Moreover, XB has found a place in medicinal chemistry and, specifically, in protein-ligand interactions. The reason is that the incorporation of a halogen onto the ligand scaffold frequently leads to enhanced drug activity. A review of the medicinal chemistry applications of XB was recently published by F. Böckler's group ${ }^{122}$ Many newly developed drugs contain a halogen atom, because the presence of halogens is beneficial in several aspects. For example, it improves oral absorption. In addition, halogens fill hydrophobic cavities in the binding site, and they facilitate crossing the bloodbrain barrier and prolong the drug lifetime. Recently, also the specific interaction of the halogens has appeared to contribute. As mentioned previously, the tunability of the XB (which is higher than that of $\mathrm{HB}$ ) represents an important advantage of $\mathrm{XB}$ and can be applied to enhance protein - ligand interactions.

$\mathrm{Lu}$ et al. studied halogen interactions in biological complexes ${ }^{257}$ both by analyzing crystallographic data and by calculations. In addition to halogen bonds, they investigated other kinds of halogen interactions. They found that oxygen and aromatic $\pi$ systems are the preferred electron donors in biomolecules. Their observations were rationalized by MP2 calculations on small complexes and by composite quantum mechanics/molecular mechanics

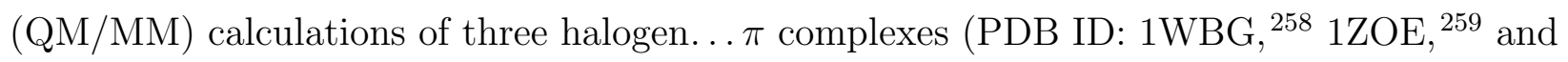
$2 \mathrm{PIT}^{(260)}$.

The energetics and character of protein-ligand halogen bonds were investigated computationally on a small series of X-ray data ${ }^{261}$ comprising the ligand binding domains of the thyroid hormone receptor, ${ }^{262}$ ADP-ribosyl transferase ART2, 263 and protein kinase CK2 259264265 (PDB IDs: 1BSX, 1GXZ, 1ZOH, 2OXD, 2OXY, and 3KXN). As noted in the study, the "best case scenarios" rather than all available motifs were selected intentionally, so that the halogen bonds studied had favorable geometric properties such as a C-X...O 
angle larger than $165^{\circ}$. A ratio of stabilization energies calculated at the Hartree-Fock and MP2 levels were calibrated by DFT-SAPT calculations of model complexes. The ratio was used to estimate the role of dispersion energy. The stabilization energies were significant, and consequently the researchers claimed that "the halogen bonds are strong interactions that play an important role in the binding of small molecule ligands to proteins." However, according to recent studies, the favorable energetics of XB may be compensated for by other contributions, such as solvation/desolvation changes.

In our recent study, we modulated $\frac{266}{2}$ aldose reductase $(\mathrm{AR})$ inhibition by halogen bond tuning. A series of AR inhibitors derived from a known AR binder exhibiting a halogen bond between its bromine atom and the oxygen atom of the Thr113 side chain of AR was investigated. The strength of the halogen bond was tuned by bromine-iodine substitution and by fluorination of the aromatic ring (Figure 21). Our conclusions were supported by Xray crystallography and IC50 measurements. Tuning the strength of the halogen bond with a monoatomic substitution decreased the IC50 value by about 1 order of magnitude (i.e., the inhibition activity increased). The composite DFT/PM6-D3H4X calculations revealed that the protein-ligand vacuum interaction energy increases upon substitution of iodine for bromine or upon the addition of electron-withdrawing fluorine atoms to the aromatic ring. However, there was no obvious relationship between the inhibition activity and the magnitude of the $\sigma$-hole or the vacuum interaction energy (Figure 21). Given the almostidentical binding poses of all of the inhibitors in crystal structures of AR-inhibitor complexes, we claim that this is because fluorination affects other properties of the ligand in addition to the halogen $\sigma$-hole, and speculate that the inhibitor solvation free energy is decisive in our case study.

To pursue our hypotheses, we designed a halogen-to-hydrogen bond substitution (Br or I atoms to an $-\mathrm{NH}_{2}$ group) in AR inhibitors. $\frac{267}{2}$ The substitution of halogen with a polar group is expected to strengthen the gas-phase-like stabilization energy (providing a suitable HB complex can be formed), but it simultaneously increases the desolvation penalty. 


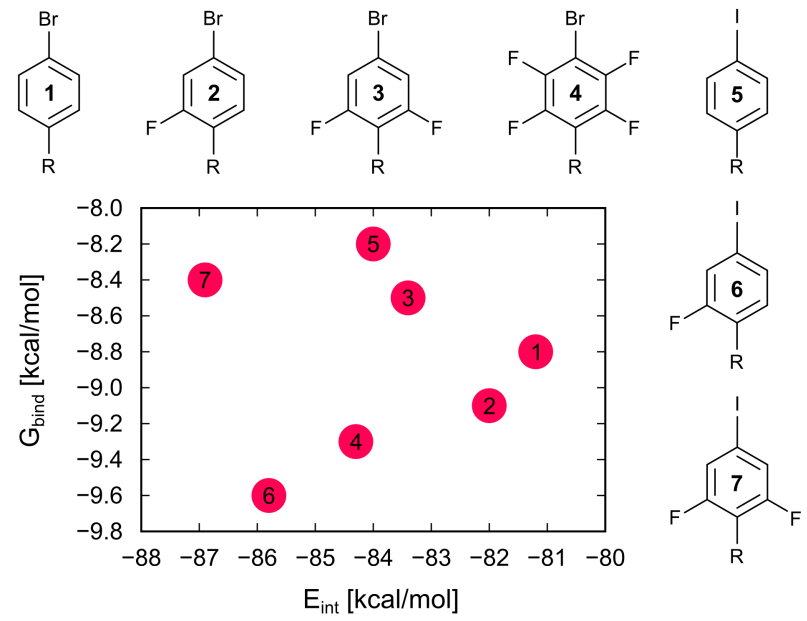

Figure 21: Structural formulas of aldose reductase inhibitors ${ }^{266}$ and the relationship between the experimental binding free energy $\left(G_{\text {bind }}\right)$ and the vacuum interaction energy $\left(E_{\text {int }}\right)$ calculated at a composite DFT/PM6-D3H4X level.

The combination of X-ray, advanced QM/SQM/MM scoring, molecular dynamics-based free energy simulations, and IC50 measurements showed that i) the geometry of the complex with XB and HB remained unchanged; ii) structurally, the XB was replaced by an HB; and iii) although the $\mathrm{HB}$ is significantly stronger than the original $\mathrm{XB}$ in a vacuum, the total binding affinity decreased due to the larger desolvation penalty of the $-\mathrm{NH}_{2}$ group. Thus, the solvent (in this case, water) plays a very important role in XB of protein-ligand complexes when competing with HB.

Carter and Shing Ho evaluated the free energy difference between XB and HB using differential scanning calorimetry of DNA junctions. ${ }^{268}$ Bromouracil was incorporated into DNA, forming an intrastrand halogen bond, which consequently determined the ternary structure of the junctions in the crystalline environment, $\stackrel{269}{ }$ consistent with the situation in solution at room temperature. The researchers claimed that the higher stabilization of the junction by halogen bonds compared to hydrogen bonds was enthalpic in origin, which was further supported by quantum chemical calculations at the MP2 level. To understand the role of solvation and steric effects, bromine was replaced with a methyl group - a chemical group of similar size. The researchers concluded that in the case of the DNA junctions, the enthalpic preference of XB over HB lies, to the first approximation, in the direct interaction 
of the electron donor and acceptor. $\stackrel{268}{26}$ Enthalpy-entropy compensation plays a role. 270

The structure-activity relationship (SAR) of two class of ligands, namely GAGA-A/benzodiazepine binders and serine protease factor Xa inhibitors, was studied by employing AM1-based field properties. $\frac{271}{}$ An important finding was that the field properties calculated at a SQM level revealed new trends that were inaccessible by conventional MM-based SAR models.

Several examples of how introduction of XB can lead to improvement in ligand activity have been reported to date: hepatitis $\mathrm{C}$ protease inhibitors; $\stackrel{272}{2}$ carboxamide inhibitors of integrin- $\alpha 4 \beta 1 ; \underline{273}$ and inhibitors of human cathepsin $L, \underline{274275}$ isoforms 1 and 4 of CDC2like kinase, $\stackrel{276}{2}$ and phosphodiesterase type 5. $\stackrel{23}{ }$ Particularly compelling results have been obtained for lead-optimization of HIV reverse transcriptase inhibitors. $\frac{2772278}{27}$ The force-fieldbased free energy perturbation Monte Carlo method identified a compound with 55 pM anti-HIV activity (EC50 to the wild-type virus). This finding was somewhat serendipitous, because although no XB-related corrections were adopted for the OPLS-AA force field at that

time, a directional chlorine-oxygen contact was predicted in the protein-ligand structure. 278 Finally, the design of a halogen-enriched fragment library by Wilcken et al. ${ }^{279}$ as a means for introduction of novel drug molecules utilizing halogen bonding should be mentioned.

\section{Concluding Remarks}

We have attempted to review theoretical approaches to the study of XB and other $\sigma$-hole interactions. The importance of both has been rising, especially in the fields of supramolecular chemistry and computer-aided drug design. We believe that in the near future, even more attention will focus on these kinds of noncovalent interactions. While research in past centuries has focused on covalent chemistry, future researchers will have to explore the world of noncovalent interactions, simply because such interactions are heavily involved in phenomena related to many recent groundbreaking discoveries. A broad arsenal of computational and experimental tools is already available to aid in understanding such phenomena. 
In our opinion, accurate $a b$ initio calculations combined with $\sigma$-hole characteristics and energy decomposition schemes are the pathway that one should follow to analyze $\sigma$-hole complexes. From the perspective of reference ab initio calculations, $\operatorname{CCSD}(\mathrm{T}) / \mathrm{CBS}$ remains the "gold standard." The density functional theory provides the M06-2X functional with its very promising results on both halogen bonded and $\sigma$-hole bonded complexes, although its use for other kinds of noncovalent interactions is not so promising. There are still, however, a few challenges to be addressed in future studies. Regarding computational methods, the largest space for improvement seems to be in the fast and efficient DFT, SQM, and MM methods. The more approximate the approach, the more balanced its contributions have to be to cover a wide range of intermolecular interactions. Focusing on a single type of noncovalent interaction might not be the best choice.

Furthermore, the role of solvent in $\sigma$-hole interactions has been studied mostly from the perspective of supramolecular chemistry ${ }^{10}$ For example, it was shown that XB vacuum interaction energies may correlate with thermodynamics in organic solvents. ${ }^{280}$ For medicinal chemistry applications, water is the exclusive solvent, and the behavior of XB in water is still poorly understood. It is well-known that the halogen atoms have electron donors in common with hydrogen bonds. Thus, the competition between halogen bonds and hydrogen bonds in biomolecule-ligand binding is largely affected by hydrogen bonding in water. We have recently shown that the thermodynamics of the two interactions are of different natures, ${ }^{267}$ but we do not think that the issue of solvation changes has been resolved. Clearly, benchmark calculations on small complexes would be welcome. Also, MM force fields, which are traditionally used to determine solvation free energies, have not yet been examined thoroughly enough in description of the thermodynamics of $\sigma$-hole complexes.

Another challenge lies in reliable predictions of the properties of $\sigma$-hole complexes. Medicinal chemistry still suffers from a lack of quantitative predictions of ligand affinities or pharmacokinetic properties. One reason might be that halogen bonding is not the decisive interaction between the ligand and its target, but makes only a minor contribution to the 
total affinity. In this case, the computational models in use fail not only due to improper description of the XB (local effect), but also due to an overall deficiency in description of the global effects.

We are quite optimistic that these questions, as well as many others, will be addressed soon, given how XB and $\sigma$-hole interactions have gained the spotlight during the last decade.

\section{Biographies}

\subsection{Michal H. Kolár}

Michal H. Kolář received his Ph.D. in 2013 from Charles University in Prague, and from the Institute of Organic Chemistry and Biochemistry, Academy of Sciences of the Czech Republic. With Pavel Hobza he focused on theoretical and computational description of noncovalent interactions. In 2012 his work on molecular modeling of halogen bonds was awarded by the French Embassy in the Czech Republic, and Sanofi-Aventis. He is a recipient of the Humboldt Research Fellowship for Postdoctoral Researchers. From 2014 he worked with Paolo Carloni on RNA-ligand recognition in Forschungszentrum Jülich, Germany. In 2016 he moved to Helmut Grubmüller's group at the Max-Planck Institute for Biophysical Chemistry, Göttingen, Germany, pursuing his interest in biomolecular recognition and computer simulations. Michal H. Kolár is active in science popularization; his efforts were recognized by the Czech Learned Society in 2012. In his spare time he likes traveling by train and dislikes tomatoes.

\subsection{Pavel Hobza}

Pavel Hobza is a holder of Distinguished Chair at the Institute of Organic Chemistry and Biochemistry, Academy of Sciences of the Czech Republic, Prague, and is a professor of chemistry at Charles University in Prague and Palacký University in Olomouc. He obtained his Ph.D. with Rudolf Zahradník in 1974 at the Academy of Sciences in Prague. After 
postdoctoral studies in Montreal (with Camille Sandorfy), Erlangen (with Paul von Rague Schleyer) and Munich (with Edward W. Schlag), he spent several periods as visiting professor in Montreal, Munich and Pohang (Republic of Korea). Dr. Hobza has authored or co-authored more than 500 papers and three books. These studies focus mainly on noncovalent interactions and their role in chemistry, biodisciplines and nanosciences. According to Thomson Reuters in 2014 and 2015, P.H. is ranked among the top 1\% of researchers with the most cited documents in the field of chemistry.

\section{Acronyms and Abbreviations}

AM1 Austin model 1

AM1-BCC AM1 with bond charge correction

au atomic units

B3LYP three-parameter Becke-Lee-Yang-Parr functional

BCP bond critical point

BSSE basis set superposition error

CBS complete basis-set limit

CC coupled-cluster theory

$\operatorname{CCSD}(\mathrm{T})$ coupled-cluster theory with iterative single and double, and perturbative triple excitations

CSD Cambridge Structural Database

CT charge transfer

DABCO diazabicyclo[2.2.2]octane 
DFT density functional theory

DFTB density-functional tight binding

DTCA 1,3-dithiole-2-thione-4-carboxylic acid

ESH explicit $\sigma$-hole

ESP electrostatic potential

FCI full configuration interaction

HB hydrogen bond

HF Hartree-Fock or hydrogen fluoride (depending on the context)

HOMO highest occupied molecular orbital

LJ Lennard-Jones

LUMO lowest unoccupied molecular orbital

MEP molecular electrostatic potential

MM molecular mechanics

MSE mean signed error

MP2 second-order Møller-Plesset perturbation theory

MUE mean unsigned error

NBO natural bond orbital

NDDP neglect of diatomic differential overlap

PDB Protein Data Bank

PM3 parameterized model 3 
PM6 parameterized model 6

PM6-D3H4X PM6 with dispersion correction (3rd geenration), hydrogen-bonding correction (4th generation) and halogen bonding correction

QCISD quadratic configuration interaction with single and double excitations

QM quantum mechanics

QTAIM quantum theory of atom in molecules

RESP restricted electrostatic potential

RMSD root-mean-square deviation

RMSE root-mean-square error

SAPT symmetry-adapted perturbation theory

SQM semiempirical quantum mechanics

XB halogen bond

\section{Acknowledgement}

We thank Dr. Fanfrlík, Dr. Lo and, Dr. Rezáč for providing us data for some of the figures. This work was part of Research Project RVO: 61388963 of the Institute of Organic Chemistry and Biochemistry, Academy of Sciences of the Czech Republic. This work was also supported by the Czech Science Foundation [P208/12/G016] and the operational program Research and Development for Innovations of the European Social Fund (CZ 1.05/2.1.00/03/0058). MHK is thankful for the support provided by the Alexander von Humboldt Foundation. 


\section{References}

(1) Desiraju, G. R.; Ho, P. S.; Kloo, L.; Legon, A. C.; Marquardt, R.; Metrangolo, P.; Politzer, P.; Resnati, G.; Rissanen, K. Definition of the halogen bond (IUPAC Recommendations 2013). Pure Appl. Chem. 2013, 85, 1711-1713.

(2) Metrangolo, P., Resnati, G., Eds. Halogen bonding: fundamentals and applications; Springer Science \& Business Media, 2007.

(3) Metrangolo, P.; Neukirch, H.; Pilati, T.; Resnati, G. Halogen bonding based recognition processes: a world parallel to hydrogen bonding. Acc. Chem. Res 2005, 38, 386-395.

(4) Politzer, P.; Lane, P.; Concha, M. C.; Ma, Y.; Murray, J. S. An overview of halogen bonding. J. Mol. Model. 2007, 13, 305-311.

(5) Metrangolo, P.; Meyer, F.; Pilati, T.; Resnati, G.; Terraneo, G. Halogen bonding in supramolecular chemistry. Angew. Chem., Int. Ed. 2008, 47, 6114-6127.

(6) Fourmigué, M. Halogen bonding: recent advances. Curr. Opin. Solid State Mater. Sci. 2009, 13, 36-45.

(7) Politzer, P.; Murray, J. S.; Clark, T. Halogen bonding: an electrostatically-driven highly directional noncovalent interaction. Phys. Chem. Chem. Phys. 2010, 12, 77487757.

(8) Legon, A. C. The halogen bond: an interim perspective. Phys. Chem. Chem. Phys. 2010, 12, 7736-7747.

(9) Erdelyi, M. Halogen bonding in solution. Chem. Soc. Rev. 2012, 41, 3547-3557.

(10) Beale, T. M.; Chudzinski, M. G.; Sarwar, M. G.; Taylor, M. S. Halogen bonding in solution: thermodynamics and applications. Chem. Soc. Rev. 2013, 42, 1667-1680. 
(11) Scholfield, M. R.; Zanden, C. M. V.; Carter, M.; Ho, P. S. Halogen bonding (Xbonding): A biological perspective. Protein Sci. 2013, 22, 139-152.

(12) Wilcken, R.; Zimmermann, M. O.; Lange, A.; Joerger, A. C.; Boeckler, F. M. Principles and applications of halogen bonding in medicinal chemistry and chemical biology. $J$. Med. Chem. 2013, 56, 1363-1388.

(13) Gilday, L. C.; Robinson, S. W.; Barendt, T. A.; Langton, M. J.; Mullaney, B. R.; Beer, P. D. Halogen Bonding in Supramolecular Chemistry. Chem. Rev. 2015, 115, 7118-7195.

(14) Scheiner, S., Ed. Noncovalent Forces; Springer International Publishing, 2015.

(15) Wolters, L. P.; Schyman, P.; Pavan, M. J.; Jorgensen, W. L.; Bickelhaupt, F. M.; Kozuch, S. The many faces of halogen bonding: a review of theoretical models and methods. Wiley Interdiscip. Rev.: Comput. Mol. Sci. 2014, 4, 523-540.

(16) Murray, J. S.; Lane, P.; Politzer, P. Expansion of the $\sigma$-hole concept. J. Mol. Model. 2009, 15, 723-729.

(17) Politzer, P.; Murray, J. S.; Clark, T. Halogen bonding and other $\sigma$-hole interactions: a perspective. Phys. Chem. Chem. Phys. 2013, 15, 11178-11189.

(18) Clark, T. $\sigma$-Holes. Wiley Interdiscip. Rev.: Comput. Mol. Sci. 2013, 3, 13-20.

(19) Politzer, P.; Murray, J. S.; Janjic, G.; Zaric, S. D. $\sigma$-hole interactions of covalentlybonded nitrogen. phosphorus and arsenic: A survey of crystal structures. Crystals 2014, 4, 12-31.

(20) Metrangolo, P.; Resnati, G. Halogen bonding: a paradigm in supramolecular chemistry. Chem. Eur. J. 2001, 7, 2511-2519.

(21) Rissanen, K. Halogen bonded supramolecular complexes and networks. CrystEngComm 2008, 10, 1107-1113. 
(22) Lu, Y.; Shi, T.; Wang, Y.; Yang, H.; Yan, X.; Luo, X.; Jiang, H.; Zhu, W. Halogen Bonding: A novel interaction for rational drug design? J. Med. Chem. 2009, 52, $2854-2862$.

(23) Xu, Z. et al. Utilization of halogen bond in lead optimization: A case study of rational design of potent phosphodiesterase type 5 (PDE5) inhibitors. J. Med. Chem. 2011, $54,5607-5611$.

(24) Bruckmann, A.; Pena, M. A.; Bolm, C. Organocatalysis through halogen-bond activation. Synlett 2008, 2008, 900-902.

(25) Kniep, F.; Jungbauer, S. H.; Zhang, Q.; Walter, S. M.; Schindler, S.; Schnapperelle, I.; Herdtweck, E.; Huber, S. M. Organocatalysis by Neutral Multidentate Halogen-Bond Donors. Angew. Chem., Int. Ed. 2013, 52, 7028-7032.

(26) Tanford, C. The Hydrophobic Effect: Formation of Micelles and Biological Membranes; Wiley, 1980.

(27) Chandler, D. Interfaces and the driving force of hydrophobic assembly. Nature 2005, 437, 640-647.

(28) Pauling, L. The nature of the chemical bond; Cornell University Press, 1960.

(29) London, F. Zur theorie und systematik der molekularkräfte. Zeitschrift für Physik 1930, 63, 245-279.

(30) Hobza, P.; Zahradník, R. Intermolecular complexes: the role of van der Waals systems in physical chemistry and in the biodisciplines; Elsevier Sciences Ltd., 1988.

(31) Clark, T.; Hennemann, M.; Murray, J. S.; Politzer, P. Halogen bonding: the $\sigma$-hole. J. Mol. Model. 2007, 13, 291-296.

(32) Shaw, D. E. et al. Anton, a Special-purpose Machine for Molecular Dynamics Simulation. Commun. ACM 2008, 51, 91-97. 
(33) Feynman, R. P. Forces in molecules. Phys. Rev. 1939, 56, 340.

(34) Politzer, P.; Riley, K. E.; Bulat, F. A.; Murray, J. S. Perspectives on halogen bonding and other $\sigma$-hole interactions: Lex parsimoniae (Occam's Razor). Comput. Theor. Chem. 2012, 998, 2-8.

(35) Politzer, P.; Murray, J. S.; Clark, T. $\sigma$-hole bonding: A physical interpretation. Top. Curr. Chem. 2014, 358, 1-25.

(36) Politzer, P.; Murray, J. S.; Clark, T. Mathematical modeling and physical reality in noncovalent interactions. J. Mol. Model. 2015, 21, 1-10.

(37) Phipps, M. J.; Fox, T.; Tautermann, C. S.; Skylaris, C. Energy decomposition analysis approaches and their evaluation on prototypical protein-drug interaction patterns. Chem. Soc. Rev. 2015, 44, 3177-3211.

(38) Stewart, R. F. On the mapping of electrostatic properties from Bragg diffraction data. Chem. Phys. Lett. 1979, 65, 335-342.

(39) Koritsanszky, T. S.; Coppens, P. Chemical applications of X-ray charge-density analysis. Chem. Rev. 2001, 101, 1583-1628.

(40) Born, M.; Oppenheimer, R. Zur quantentheorie der molekeln. Ann. Phys. 1927, 389, 457-484.

(41) Bader, R. F.; Carroll, M. T.; Cheeseman, J. R.; Chang, C. Properties of atoms in molecules: atomic volumes. J. Am. Chem. Soc. 1987, 109, 7968-7979.

(42) Cox, S.; Williams, D. Representation of the molecular electrostatic potential by a net atomic charge model. J. Comput. Chem. 1981, 2, 304-323.

(43) Pullman, A.; Pullman, B. Molecular electrostatic potential of the nucleic acids. $Q$. Rev. Biophys. 1981, 14, 289-380. 
(44) Politzer, P.; Truhlar, D. G. Chemical applications of atomic and molecular electrostatic potentials: reactivity, structure, scattering, and energetics of organic, inorganich, and biological systems; Springer Science \& Business Media, 2013.

(45) Brinck, T.; Murray, J. S.; Politzer, P. Surface electrostatic potentials of halogenated methanes as indicators of directional intermolecular interactions. Int. J. Quantum Chem. 1992, 44, 57-64.

(46) Roothaan, C. C. J. New developments in molecular orbital theory. Rev. Mod. Phys. 1951, 23, 69.

(47) Ramasubbu, N.; Parthasarathy, R.; Murray-Rust, P. Angular preferences of intermolecular forces around halogen centers: preferred directions of approach of electrophiles and nucleophiles around carbon-halogen bond. J. Am. Chem. Soc. 1986, $108,4308-4314$.

(48) Hassel, O.; Hvoslef, J. The structure of bromine 1, 4-dioxanate. Acta Chem. Scand. 1954, 8, 873-873.

(49) Hassel, O.; Strømme, K. Structure of the crystalline compound benzene-bromine (1-1). Acta Chem. Scand. 1958, 12, 1146-1147.

(50) Hassel, O.; Strømme, K. Crystal Structure of the 1: 1 Addition Compound Formed by Acetone and Bromine. Acta Chem. Scand. 1959, 13, 275-280.

(51) Collin, R. L. The crystal strucutre of solid chlorine. Acta Cryst. 1956, 9, 537-537.

(52) Donohue, J.; Goodman, S. H. Interatomic distances in solid chlorine. Acta Cryst. $1965,18,568-569$.

(53) Nyburg, S. Polar flattening: non-spherical effective shapes of atoms in crystals. Acta Crystallogr., Sect. A: Cryst. Phys., Diffr., Theor. Gen. Crystallogr. 1979, 35, 641-645. 
(54) Murray, J. S.; Riley, K. E.; Politzer, P.; Clark, T. Directional weak intermolecular interactions: $\sigma$-hole bonding. Aust. J. Chem. 2010, 63, 1598-1607.

(55) Wang, W.; Ji, B.; Zhang, Y. Chalcogen bond: a sister noncovalent bond to halogen bond. J. Phys. Chem. A 2009, 113, 8132-8135.

(56) Brezgunova, M. E.; Lieffrig, J.; Aubert, E.; Dahaoui, S.; Fertey, P.; Lebègue, S.; Angyan, J. G.; Fourmigué, M.; Espinosa, E. Chalcogen bonding: experimental and theoretical determinations from electron density analysis Geometrical preferences driven by electrophilic-nucleophilic interactions. Cryst. Growth Des. 2013, 13, 3283-3289.

(57) Del Bene, J. E.; Alkorta, I.; Sanchez-Sanz, G.; Elguero, J. Structures, Energies, Bonding, and NMR Properties of Pnicogen Complexes $\mathrm{H}_{2} \mathrm{XP}$ : $\mathrm{NXH}_{2}\left(\mathrm{X}: \mathrm{H}, \mathrm{CH}_{3}, \mathrm{NH}_{2}, \mathrm{OH}\right.$, F, Cl). J. Phys. Chem. A 2011, 115, 13724-13731.

(58) Zahn, S.; Frank, R.; Hey-Hawkins, E.; Kirchner, B. Pnicogen bonds: a new molecular linker? Chem. Eur. J. 2011, 17, 6034-6038.

(59) Scheiner, S. The pnicogen bond: its relation to hydrogen, halogen, and other noncovalent bonds. Acc. Chem. Res 2012, 46, 280-288.

(60) Bauzá, A.; Mooibroek, T. J.; Frontera, A. Tetrel-Bonding Interaction: Rediscovered Supramolecular Force? Angew. Chem., Int. Ed. 2013, 125, 12543-12547.

(61) Grabowski, S. J. Tetrel bond- $\sigma$-hole bond as a preliminary stage of the SN 2 reaction. Phys. Chem. Chem. Phys. 2014, 16, 1824-1834.

(62) Bauzá, A.; Frontera, A. Aerogen Bonding Interaction: A New Supramolecular Force? Angew. Chem., Int. Ed. 2015, 54, 7340-7343.

(63) Fanfrlík, J.; Přáda, A.; Padělková, Z.; Pecina, A.; Macháček, J.; Lepš́́k, M.; Holub, J.; Růžička, A.; Hnyk, D.; Hobza, P. The dominant role of chalcogen bonding in the crystal packing of 2D/3D aromatics. Angew. Chem., Int. Ed. 2014, 53, 10139-10142. 
(64) Lo, R.; Fanfrlík, J.; Lepšík, M.; Hobza, P. The properties of substituted 3D-aromatic neutral carboranes: the potential for $\sigma$-hole bonding. Phys. Chem. Chem. Phys. 2015, 17, 20814-20821.

(65) Kolář, M.; Hostaš, J.; Hobza, P. The strength and directionality of a halogen bond are co-determined by the magnitude and size of the $\sigma$-hole. Phys. Chem. Chem. Phys. 2014, 16, 9987-9996.

(66) Kolář, M. H.; Carloni, P.; Hobza, P. Statistical analysis of $\sigma$-holes: a novel complementary view on halogen bonding. Phys. Chem. Chem. Phys. 2014, 16, 19111-19114.

(67) Kolář, M. H.; Deepa, P.; Ajani, H.; Pecina, A.; Hobza, P. Characteristics of a sigmaHole and the Nature of a Halogen Bond. Top. Curr. Chem. 2015, 359, 1-25.

(68) Murray, J. S.; Politzer, P. Correlations between the solvent hydrogen-bond-donating parameter .alpha. and the calculated molecular surface electrostatic potential. J. Org. Chem. 1991, 56, 6715-6717.

(69) Řezáč, J.; Riley, K. E.; Hobza, P. Benchmark calculations of noncovalent interactions of halogenated molecules. J. Chem. Theory Comput. 2012, 8, 4285-4292.

(70) Lu, Y.; Zou, J.; Yu, Q.; Jiang, Y.; Zhao, W. Ab initio investigation of halogen bonding interactions involving fluorine as an electron acceptor. Chem. Phys. Lett. 2007, 449, 6-10.

(71) Metrangolo, P.; Murray, J. S.; Pilati, T.; Politzer, P.; Resnati, G.; Terraneo, G. The fluorine atom as a halogen bond donor, viz a positive site. CrystEngComm 2011, 13, 6593-6596.

(72) Legon, A. C. Prereactive complexes of dihalogens XY with Lewis bases B in the gas phase: a systematic case for the halogen analogue BXY of the hydrogen bond BHX. Angew. Chem., Int. Ed. 1999, 38, 2686-2714. 
(73) Metrangolo, P.; Murray, J. S.; Pilati, T.; Politzer, P.; Resnati, G.; Terraneo, G. Fluorine-centered halogen bonding: a factor in recognition phenomena and reactivity. Cryst. Growth Des. 2011, 11, 4238-4246.

(74) Eskandari, K.; Lesani, M. Does fluorine participate in halogen bonding? Chem. Eur. J. 2015, 21, 4739-4746.

(75) Riley, K. E.; Murray, J. S.; Politzer, P.; Concha, M. C.; Hobza, P. BrO complexes as probes of factors affecting halogen bonding: interactions of bromobenzenes and bromopyrimidines with acetone. J. Chem. Theory Comput. 2008, 5, 155-163.

(76) Riley, K. E.; Murray, J. S.; Fanfrlík, J.; Řezáč, J.; Solá, R. J.; Concha, M. C.; Ramos, F. M.; Politzer, P. Halogen bond tunability I: the effects of aromatic fluorine substitution on the strengths of halogen-bonding interactions involving chlorine, bromine, and iodine. J. Mol. Model. 2011, 17, 3309-3318.

(77) Riley, K. E.; Murray, J. S.; Fanfrlík, J.; Řezáč, J.; Solá, R. J.; Concha, M. C.; Ramos, F. M.; Politzer, P. Halogen bond tunability II: the varying roles of electrostatic and dispersion contributions to attraction in halogen bonds. J. Mol. Model. 2013, 19, 4651-4659.

(78) Voth, A. R.; Khuu, P.; Oishi, K.; Ho, P. S. Halogen bonds as orthogonal molecular interactions to hydrogen bonds. Nature Chem. 2009, 1, 74-79.

(79) Hennemann, M.; Murray, J. S.; Politzer, P.; Riley, K. E.; Clark, T. Polarizationinduced $\sigma$-holes and hydrogen bonding. J. Mol. Model. 2012, 18, 2461-2469.

(80) Fiedler, L.; Gao, J.; Truhlar, D. G. Polarized Molecular Orbital Model Chemistry 1 Ab Initio Foundations. J. Chem. Theory Comput. 2011, 7, 852-856.

(81) Clark, T.; Politzer, P.; Murray, J. S. Correct electrostatic treatment of noncovalent 
interactions: the importance of polarization. Wiley Interdiscip. Rev.: Comput. Mol. Sci. 2015, 5, 169-177.

(82) Weigend, F.; Ahlrichs, R. Balanced basis sets of split valence, triple zeta valence and quadruple zeta valence quality for $\mathrm{H}$ to Rn: design and assessment of accuracy. Phys. Chem. Chem. Phys. 2005, 7, 3297-3305.

(83) Pople, J. A.; Head-Gordon, M.; Raghavachari, K. Quadratic configuration interaction A general technique for determining electron correlation energies. J. Chem. Phys. 1987, 87, 5968-5975.

(84) Frisch, M. J. et al. Gaussian 09, Revision D.01. Gaussian, Inc., Wallingford CT, 2009.

(85) Schuchardt, K. L.; Didier, B. T.; Elsethagen, T.; Sun, L.; Gurumoorthi, V.; Chase, J.; Li, J.; Windus, T. L. Basis set exchange: a community database for computational sciences. J. Chem. Inf. Model. 2007, 47, 1045-1052.

(86) Møller, C.; Plesset, M. S. Note on an approximation treatment for many-electron systems. Phys. Rev. 1934, 46, 618.

(87) Yanai, T.; Tew, D. P.; Handy, N. C. A new hybrid exchange-correlation functional using the Coulomb-attenuating method (CAM-B3LYP). Chem. Phys. Lett. 2004, 393, $51-57$.

(88) Rappoport, D.; Furche, F. Property-optimized Gaussian basis sets for molecular response calculations. J. Chem. Phys. 2010, 133, 134105.

(89) Valerio, G.; Raos, G.; Meille, S. V.; Metrangolo, P.; Resnati, G. Halogen bonding in fluoroalkylhalides: a quantum chemical study of increasing fluorine substitution. $J$. Phys. Chem. A 2000, 104, 1617-1620.

(90) Lu, Y.; Zou, J.; Wang, Y.; Jiang, Y.; Yu, Q. Ab initio investigation of the complexes between bromobenzene and several electron donors: some insights into the magnitude 
and nature of halogen bonding interactions. J. Phys. Chem. A 2007, 111, 1078110788.

(91) Shields, Z. P.; Murray, J. S.; Politzer, P. Directional tendencies of halogen and hydrogen bonds. Int. J. Quantum Chem. 2010, 110, 2823-2832.

(92) Politzer, P.; Murray, J. S. Halogen bonding: an interim discussion. ChemPhysChem 2013, 14, 278-294.

(93) Kozuch, S.; Martin, J. M. Halogen bonds: Benchmarks and theoretical analysis. J. Chem. Theory Comput. 2013, 9, 1918-1931.

(94) Deepa, P.; Sedlak, R.; Hobza, P. On the origin of the substantial stabilisation of the electron-donor 1, 3-dithiole-2-thione-4-carboxyclic acidI2 and DABCOI2 complexes. Phys. Chem. Chem. Phys. 2014, 16, 6679-6686.

(95) Riley, K. E.; Hobza, P. Investigations into the nature of halogen bonding including symmetry adapted perturbation theory analyses. J. Chem. Theory Comput. 2008, 4, $232-242$.

(96) Riley, K. E.; Hobza, P. The relative roles of electrostatics and dispersion in the stabilization of halogen bonds. Phys. Chem. Chem. Phys. 2013, 15, 17742-17751.

(97) Jeziorski, B.; Moszynski, R.; Szalewicz, K. Perturbation theory approach to intermolecular potential energy surfaces of van der Waals complexes. Chem. Rev. 1994, 94, 1887-1930.

(98) Stone, A. J.; J., M. A. Charge-transfer in symmetry-adapted perturbation theory. Chem. Phys. Lett. 2009, 473, 201-205.

(99) Reed, A. E.; Curtiss, L. A.; Weinhold, F. Intermolecular interactions from a natural bond orbital, donor-acceptor viewpoint. Chem. Rev. 1988, 88, 899-926. 
(100) Heßelmann, A.; Jansen, G. First-order intermolecular interaction energies from Kohn-Sham orbitals. Chem. Phys. Lett. 2002, 357, 464-470.

(101) Heßelmann, A.; Jansen, G. Intermolecular induction and exchange-induction energies from coupled-perturbed Kohn-Sham density functional theory. Chem. Phys. Lett. 2002, 362, 319-325.

(102) Misquitta, A. J.; Szalewicz, K. Intermolecular forces from asymptotically corrected density functional description of monomers. Chem. Phys. Lett. 2002, 357, 301-306.

(103) Jansen, G.; Heßelmann, A. Comment on "Using Kohn-Sham orbitals in symmetryadapted perturbation theory to investigate intermolecular interactions". J. Phys. Chem. A 2001, 105, 11156-11157.

(104) Heßelmann, A.; Jansen, G. Intermolecular dispersion energies from time-dependent density functional theory. Chem. Phys. Lett. 2003, 367, 778-784.

(105) Heßelmann, A.; Jansen, G.; Schütz, M. Density-functional theory-symmetry-adapted intermolecular perturbation theory with density fitting: A new efficient method to study intermolecular interaction energies. J. Chem. Phys. 2005, 122, 014103.

(106) Heßelmann, A.; Jansen, G.; Schütz, M. Interaction energy contributions of H-bonded and stacked structures of the AT and GC DNA base pairs from the combined density functional theory and intermolecular perturbation theory approach. J. Am. Chem. Soc. 2006, 128, 11730-11731.

(107) Podeszwa, R.; Bukowski, R.; Szalewicz, K. Density-fitting method in symmetryadapted perturbation theory based on Kohn-Sham description of monomers. J. Chem. Theory Comput. 2006, 2, 400-412.

(108) Williams, H. L.; Chabalowski, C. F. Using Kohn-Sham orbitals in symmetry-adapted 
perturbation theory to investigate intermolecular interactions. J. Phys. Chem. A 2001, $105,646-659$.

(109) El Kerdawy, A.; Murray, J. S.; Politzer, P.; Bleiziffer, P.; Heßelmann, A.; Görling, A.; Clark, T. Directional noncovalent interactions: Repulsion and dispersion. J. Chem. Theory Comput. 2013, 9, 2264-2275.

(110) Sedlák, R.; Kolář, M. H.; Hobza, P. Polar Flattening and the strenght of halogen bonding J. Chem. Theory Comput. J. Chem. Theory Comput. 2015, O(0), 00-00.

(111) Bleiholder, C.; Werz, D. B.; Köppel, H.; Gleiter, R. Theoretical investigations on chalcogen-chalcogen interactions: What makes these nonbonded interactions bonding? J. Am. Chem. Soc. 2006, 128, 2666-2674.

(112) Scheiner, S. Detailed comparison of the pnicogen bond with chalcogen, halogen, and hydrogen bonds. Int. J. Quantum Chem. 2013, 113, 1609-1620.

(113) Pecina, A.; Lepš́k, M.; Hnyk, D.; Hobza, P.; Fanfrlík, J. Chalcogen and Pnicogen Bonds in Complexes of Neutral Icosahedral and Bicapped Square-Antiprismatic Heteroboranes. J. Phys. Chem. A 2015, 119, 1388-1395.

(114) Setiawan, D.; Kraka, E.; Cremer, D. Strength of the Pnicogen Bond in Complexes Involving Group Va Elements N, P, and As. J. Phys. Chem. A 2014, 119, 1642-1656.

(115) Munusamy, E.; Sedlak, R.; Hobza, P. On the Nature of the Stabilization of BenzeneDihalogen and BenzeneDinitrogen Complexes: CCSD(T)/CBS and DFT-SAPT Calculations. ChemPhysChem 2011, 12, 3253-3261.

(116) Sedlak, R.; Deepa, P.; Hobza, P. Why Is the L-Shaped Structure of X2X2 (X= F, Cl, Br, I) Complexes More Stable Than Other Structures? J. Phys. Chem. A 2014, 118, 3846-3855.

(117) Bader, R. F. Atoms in molecules. Acc. Chem. Res 1985, 18, 9-15. 
(118) Matta, C. F.; Hernández-Trujillo, J.; Tang, T.; Bader, R. F. Hydrogen-hydrogen bonding: a stabilizing interaction in molecules and crystals. Chem. Eur. J. 2003, 9, 1940-1951.

(119) Poater, J.; Solà, M.; Bickelhaupt, F. M. Hydrogen-Hydrogen Bonding in Planar Biphenyl, Predicted by Atoms-In-Molecules Theory, Does Not Exist. Chem. Eur. J. 2006, 12, 2889-2895.

(120) Eskandari, K.; Zariny, H. Halogen bonding: a lump-hole interaction. Chem. Phys. Lett. 2010, 492, 9-13.

(121) Grabowski, S. J. QTAIM characteristics of halogen bond and related interactions. $J$. Phys. Chem. A 2012, 116, 1838-1845.

(122) Gavezzotti, A. Non-conventional bonding between organic molecules The 'halogen bond'in crystalline systems. Mol. Phys. 2008, 106, 1473-1485.

(123) Allen, F.; Lommerse, J.; Hoy, V.; Howard, J.; Desiraju, G. Halogen...O (nitro) supramolecular synthon in crystal engineering: a combined crystallographic database and ab initio molecular orbital study. Acta Crystallogr., Sect. B: Struct. Sci. 1997, 53, 1006-1016.

(124) Palusiak, M. On the nature of halogen bond-The Kohn-Sham molecular orbital approach. J. Mol. Struct.: THEOCHEM 2010, 945, 89-92.

(125) Wolters, L. P.; Bickelhaupt, F. M. Halogen bonding versus hydrogen bonding: a molecular orbital perspective. ChemistryOpen 2012, 1, 96-105.

(126) Pinter, B.; Nagels, N.; Herrebout, W. A.; De Proft, F. Halogen bonding from a hard and soft acids and bases perspective: investigation by using density functional theory reactivity indices. Chem. Eur. J. 2013, 19, 519-530. 
(127) Čížek, J. On the correlation problem in atomic and molecular systems Calculation of wavefunction components in Ursell-type expansion using quantum-field theoretical methods. J. Chem. Phys. 1966, 45, 4256-4266.

(128) Bartlett, R. J.; Musiał, M. Coupled-cluster theory in quantum chemistry. Rev. Mod. Phys. 2007, 79, 291.

(129) Paldus, J.; Č́žzek, J.; Shavitt, I. Correlation Problems in Atomic and Molecular Systems IV Extended Coupled-Pair Many-Electron Theory and Its Application to the B H 3 Molecule. Phys. Rev. A 1972, 5, 50.

(130) Szabo, A.; Ostlund, N. S. Modern quantum chemistry; Dover Publications, 1996.

(131) Halkier, A.; Helgaker, T.; Jørgensen, P.; Klopper, W.; Koch, H.; Olsen, J.; Wilson, A. K. Basis-set convergence in correlated calculations on Ne, N 2, and H 2 O. Chem. Phys. Lett. 1998, 286, 243-252.

(132) Řezáč, J.; Hobza, P. Describing noncovalent interactions beyond the common approximations: How accurate is the "gold standard," $\operatorname{CCSD}(\mathrm{T})$ at the complete basis set limit? J. Chem. Theory Comput. 2013, 9, 2151-2155.

(133) Boys, S. F.; Bernardi, F. D. The calculation of small molecular interactions by the differences of separate total energies Some procedures with reduced errors. Mol. Phys. 1970, 19, 553-566.

(134) Koch, H.; Fernández, B.; Christiansen, O. The benzene-argon complex: A ground and excited state ab initio study. J. Chem. Phys. 1998, 108, 2784-2790.

(135) Hobza, P.; Šponer, J. Toward true DNA base-stacking energies: MP2, CCSD(T), and complete basis set calculations. J. Am. Chem. Soc. 2002, 124, 11802-11808.

(136) Sinnokrot, M. O.; Valeev, E. F.; Sherrill, C. D. Estimates of the ab initio limit for $\pi-\pi$ interactions: The benzene dimer. J. Am. Chem. Soc. 2002, 124, 10887-10893. 
(137) Karpfen, A. Charge-transfer complexes between NH3 and the halogens F2, ClF, and Cl2: an ab initio study on the intermolecular interaction. J. Phys. Chem. A 2000, 104, 6871-6879.

(138) Řezáč, J.; Riley, K. E.; Hobza, P. S66: A well-balanced database of benchmark interaction energies relevant to biomolecular structures. J. Chem. Theory Comput. 2011, 7, 2427-2438.

(139) Bauzá, A.; Alkorta, I.; Frontera, A.; Elguero, J. On the reliability of pure and hybrid DFT methods for the evaluation of halogen, chalcogen, and pnicogen bonds involving anionic and neutral electron donors. J. Chem. Theory Comput. 2013, 9, 5201-5210.

(140) Řezáč, J.; Riley, K. E.; Hobza, P. Evaluation of the performance of post-Hartree-Fock methods in terms of intermolecular distance in noncovalent complexes. J. Comput. Chem. 2012, 33, 691-694.

(141) Kolář, M.; Hobza, P. Accurate Theoretical Determination of the Structure of Aromatic Complexes Is Complicated: The Phenol Dimer and Phenol Methanol Cases. J. Phys. Chem. A 2007, 111, 5851-5854.

(142) Riley, K. E.; Hobza, P. Assessment of the MP2 method, along with several basis sets, for the computation of interaction energies of biologically relevant hydrogen bonded and dispersion bound complexes. J. Phys. Chem. A 2007, 111, 8257-8263.

(143) Cybulski, S. M.; Lytle, M. L. The origin of deficiency of the supermolecule second-order Møller-Plesset approach for evaluating interaction energies. J. Chem. Phys. 2007, 12\%, 141102.

(144) Riley, K. E.; Platts, J. A.; Řezáč, J.; Hobza, P.; Hill, J. G. Assessment of the Performance of MP2 and MP2 Variants for the Treatment of Noncovalent Interactions. $J$. Phys. Chem. A 2012, 116, 4159-4169. 
(145) Grimme, S. Improved second-order Møller-Plesset perturbation theory by separate scaling of parallel-and antiparallel-spin pair correlation energies. J. Chem. Phys. 2003, 118, 9095-9102.

(146) Distasio Jr, R. A.; Head-Gordon, M. Optimized spin-component scaled second-order Møller-Plesset perturbation theory for intermolecular interaction energies. Mol. Phys. 2007, 105, 1073-1083.

(147) Takatani, T.; Hohenstein, E. G.; Sherrill, C. D. Improvement of the coupled-cluster singles and doubles method via scaling same-and opposite-spin components of the double excitation correlation energy. J. Chem. Phys. 2008, 128, 124111.

(148) Pitoňák, M.; Řezáč, J.; Hobza, P. Spin-component scaled coupled-clusters singles and doubles optimized towards calculation of noncovalent interactions. Phys. Chem. Chem. Phys. 2010, 12, 9611-9614.

(149) Pitoňák, M.; Neogrády, P.; Černý, J.; Grimme, S.; Hobza, P. Scaled MP3 NonCovalent Interaction Energies Agree Closely with Accurate CCSD(T) Benchmark Data. ChemPhysChem 2009, 10, 282-289.

(150) Grimme, S.; Ehrlich, S.; Goerigk, L. Effect of the damping function in dispersion corrected density functional theory. J. Comput. Chem. 2011, 32, 1456-1465.

(151) Řezáč, J.; Huang, Y.; Hobza, P.; Beran, G. J. Benchmark Calculations of Three-Body Intermolecular Interactions and the Performance of Low-Cost Electronic Structure Methods. J. Chem. Theory Comput. 2015, 11, 3065-3079.

(152) Perdew, J. P.; Schmidt, K. AIP Conference Proceedings; IOP INSTITUTE OF PHYSICS PUBLISHING LTD, 2001.

(153) Zhao, Y.; Truhlar, D. G. The M06 suite of density functionals for main group thermochemistry, thermochemical kinetics, noncovalent interactions, excited states, and 
transition elements: two new functionals and systematic testing of four M06-class functionals and 12 other functionals. Theor. Chem. Acc. 2008, 120, 215-241.

(154) Boese, A. D.; Martin, J. M. Development of density functionals for thermochemical kinetics. J. Chem. Phys. 2004, 121, 3405-3416.

(155) Chai, J.; Head-Gordon, M. Long-range corrected hybrid density functionals with damped atom-atom dispersion corrections. Phys. Chem. Chem. Phys. 2008, 10, 66156620.

(156) Grimme, S. Accurate description of van der Waals complexes by density functional theory including empirical corrections. J. Comput. Chem. 2004, 25, 1463-1473.

(157) Otero-de-la Roza, A.; Johnson, E.; DiLabio, G. Halogen bonding from dispersioncorrected density-functional theory: the role of delocalization error. J. Chem. Theory Comput. 2014, 10, 5436-5447.

(158) Becke, A. D.; Johnson, E. R. Exchange-hole dipole moment and the dispersion interaction. J. Chem. Phys. 2005, 122, 154104.

(159) Bauzá, A.; Alkorta, I.; Frontera, A.; Elguero, J. Supplementary Information of: On the reliability of pure and hybrid DFT methods for the evaluation of halogen, chalcogen, and pnicogen bonds involving anionic and neutral electron donors. J. Chem. Theory Comput. 2013, 9, 5201-5210.

(160) Pople, J. A.; Santry, D. P.; Segal, G. A. Approximate Self-Consistent Molecular Orbital Theory I Invariant Procedures. J. Chem. Phys. 1965, 43, S129-S135.

(161) Pople, J.; Beveridge, D.; Dobosh, P. Approximate Self-Consistent Molecular-Orbital Theory V Intermediate Neglect of Differential Overlap. J. Chem. Phys. 1967, 47, 2026-2033. 
(162) Dewar, M. J.; Zoebisch, E. G.; Healy, E. F.; Stewart, J. J. Development and use of quantum mechanical molecular models 76 AM1: a new general purpose quantum mechanical molecular model. J. Am. Chem. Soc. 1985, 10\%, 3902-3909.

(163) Stewart, J. J. Optimization of parameters for semiempirical methods I Method. J. Comput. Chem. 1989, 10, 209-220.

(164) Stewart, J. J. Optimization of parameters for semiempirical methods V: modification of NDDO approximations and application to 70 elements. J. Mol. Model. 2007, 13, $1173-1213$.

(165) Elstner, M.; Porezag, D.; Jungnickel, G.; Elsner, J.; Haugk, M.; Frauenheim, T.; Suhai, S.; Seifert, G. Self-consistent-charge density-functional tight-binding method for simulations of complex materials properties. Phys. Rev. B 1998, 58, 7260.

(166) Gaus, M.; Cui, Q.; Elstner, M. DFTB3: extension of the self-consistent-charge densityfunctional tight-binding method (SCC-DFTB). J. Chem. Theory Comput. 2011, 7, $931-948$.

(167) Kubař, T.; Bodrog, Z.; Gaus, M.; Köhler, C.; Aradi, B.; Frauenheim, T.; Elstner, M. Parametrization of the SCC-DFTB Method for Halogens. J. Chem. Theory Comput. 2013, 9, 2939-2949.

(168) Kubillus, M.; Kubař, T.; Gaus, M.; Řezáč, J.; Elstner, M. Parameterization of the DFTB3 Method for Br, Ca, Cl, F, I, K, and Na in Organic and Biological Systems. J. Chem. Theory Comput. 2014, 11, 332-342.

(169) Li, A.; Muddana, H. S.; Gilson, M. K. Quantum mechanical calculation of noncovalent interactions: A large-scale evaluation of PMx, DFT, and SAPT approaches. J. Chem. Theory Comput. 2014, 10, 1563-1575. 
(170) Řezáč, J.; Hobza, P. Advanced corrections of hydrogen bonding and dispersion for semiempirical quantum mechanical methods. J. Chem. Theory Comput. 2011, 8, 141151.

(171) Lepšík, M.; Rezáǎ, J.; Kolář, M.; Pecina, A.; Hobza, P.; Fanfrlík, J. The semiempirical quantum mechanical scoring function for in silico drug design. ChemPlusChem 2013, 78, 921-931.

(172) Řezáč, J.; Hobza, P. A halogen-bonding correction for the semiempirical PM6 method. Chem. Phys. Lett. 2011, 506, 286-289.

(173) Dobeš, P.; Rezáč, J.; Fanfrlík, J.; Otyepka, M.; Hobza, P. Semiempirical quantum mechanical method PM6-DH2X describes the geometry and energetics of CK2-inhibitor complexes involving halogen bonds well, while the empirical potential fails. J. Phys. Chem. B 2011, 115, 8581-8589.

(174) Weber, W.; Thiel, W. Orthogonalization corrections for semiempirical methods. Theor. Chem. Acc. 2000, 103, 495-506.

(175) Rocha, G. B.; Freire, R. O.; Simas, A. M.; Stewart, J. J. RM1: A reparameterization of AM1 for H, C, N, O, P, S, F, Cl, Br, and I. J. Comput. Chem. 2006, 27, 1101-1111.

(176) Stewart, J. J. Application of localized molecular orbitals to the solution of semiempirical self-consistent field equations. Int. J. Quantum Chem. 1996, 58, 133-146.

(177) Stewart, J. J. MOPAC2009. Stewart Computational Chemistry. Colorado Springs, CO, USA, 2009.

(178) Hernandes, M. Z.; Cavalcanti, S. M. T.; Moreira, D. R. M.; de Azevedo, J.; Filgueira, W.; Leite, A. C. L. Halogen atoms in the modern medicinal chemistry: hints for the drug design. Curr. Drug Targets 2010, 11, 303-314. 
(179) Karplus, M.; McCammon, J. A. Molecular dynamics simulations of biomolecules. Nat. Struct. Mol. Biol. 2002, 9, 646-652.

(180) Verlet, L. Computer experiments on classical fluids I Thermodynamical properties of Lennard-Jones molecules. Phys. Rev. 1967, 159, 98-103.

(181) Nicolas, J.; Gubbins, K.; Streett, W.; Tildesley, D. Equation of state for the LennardJones fluid. Mol. Phys. 1979, 37, 1429-1454.

(182) Besler, B. H.; Merz, K. M.; Kollman, P. A. Atomic charges derived from semiempirical methods. J. Comput. Chem. 1990, 11, 431-439.

(183) Wiberg, K. B.; Rablen, P. R. Comparison of atomic charges derived via different procedures. J. Comput. Chem. 1993, 14, 1504-1518.

(184) Sigfridsson, E.; Ryde, U. Comparison of methods for deriving atomic charges from the electrostatic potential and moments. J. Comput. Chem. 1998, 19, 377-395.

(185) Bayly, C. I.; Cieplak, P.; Cornell, W.; Kollman, P. A. A well-behaved electrostatic potential based method using charge restraints for deriving atomic charges: the RESP model. J. Phys. Chem. 1993, 97, 10269-10280.

(186) Jakalian, A.; Bush, B. L.; Jack, D. B.; Bayly, C. I. Fast, efficient generation of highquality atomic Charges AM1-BCC model: I Method. J. Comput. Chem. 2000, 21, $132-146$.

(187) Jakalian, A.; Jack, D. B.; Bayly, C. I. Fast, efficient generation of high-quality atomic charges AM1-BCC model: II Parameterization and validation. J. Comput. Chem. 2002, 23, 1623-1641.

(188) Kramer, C.; Spinn, A.; Liedl, K. R. Charge anisotropy: Where atomic multipoles matter most. J. Chem. Theory Comput. 2014, 10, 4488-4496. 
(189) Politzer, P.; Murray, J. S.; Concha, M. C. $\sigma$-hole bonding between like atoms; a fallacy of atomic charges. J. Mol. Model. 2008, 14, 659-665.

(190) Vinter, J. G. Extended electron distributions applied to the molecular mechanics of some intermolecular interactions. J. Comput.-Aided Mol. Des. 1994, 8, 653-668.

(191) Ibrahim, M. A. Molecular mechanical study of halogen bonding in drug discovery. $J$. Comput. Chem. 2011, 32, 2564-2574.

(192) Rendine, S.; Pieraccini, S.; Forni, A.; Sironi, M. Halogen bonding in ligand-receptor systems in the framework of classical force fields. Phys. Chem. Chem. Phys. 2011, 13, $19508-19516$.

(193) Kolář, M.; Hobza, P. On extension of the current biomolecular empirical force field for the description of halogen bonds. J. Chem. Theory Comput. 2012, 8, 1325-1333.

(194) Jorgensen, W. L.; Schyman, P. Treatment of halogen bonding in the OPLS-AA force field: application to potent anti-HIV agents. J. Chem. Theory Comput. 2012, 8, 38953901.

(195) Dixon, R. W.; Kollman, P. A. Advancing beyond the atom-centered model in additive and nonadditive molecular mechanics. J. Comput. Chem. 1997, 18, 1632-1646.

(196) van der Spoel, D.; Lindahl, E.; Hess, B.; development team, G. GROMACS User Manual version 4.6.5. www.gromacs.org, 2013.

(197) Berendsen, H. J.; Postma, J. P.; van Gunsteren, W. F.; Hermans, J. In Intermolecular forces; Pullmann, B., Ed.; Springer Netherlands, 1981; pp 331-342.

(198) Jorgensen, W. L.; Chandrasekhar, J.; Madura, J. D.; Impey, R. W.; Klein, M. L. Comparison of simple potential functions for simulating liquid water. J. Chem. Phys. 1983, 79, 926-935. 
(199) Pronk, S.; Páll, S.; Schulz, R.; Larsson, P.; Bjelkmar, P.; Apostolov, R.; Shirts, M. R.; Smith, J. C.; Kasson, P. M.; van der Spoel, D.; Hess, E., B.and Lindahl GROMACS 4.5: a high-throughput and highly parallel open source molecular simulation toolkit. Bioinformatics 2013,

(200) Pall, S.; Abraham, M. J.; Kutzner, C.; Hess, B.; Lindahl, E. Tackling exascale software challenges in molecular dynamics simulations with GROMACS. Solving Software Challenges for Exascale, 2014.

(201) Case, D. A. et al. Amber 14. 2014.

(202) Cornell, W. D.; Cieplak, P.; Bayly, C. I.; Gould, I. R.; Merz, K. M.; Ferguson, D. M.; Spellmeyer, D. C.; Fox, T.; Caldwell, J. W.; Kollman, P. A. A second generation force field for the simulation of proteins, nucleic acids, and organic molecules. J. Am. Chem. Soc. 1995, 117, 5179-5197.

(203) Wang, J.; Wolf, R. M.; Caldwell, J. W.; Kollman, P. A.; Case, D. A. Development and testing of a general amber force field. J. Comput. Chem. 2004, 25, 1157-1174.

(204) Titov, O. I.; Shulga, D. A.; Palyulin, V. A.; Zefirov, N. S. Perspectives of Halogen Bonding Description in Scoring Functions and QSAR/QSPR: Substituent Effects in Aromatic Core. Mol. Inf. 2015, 34, 404-416.

(205) Torii, H.; Yoshida, M. Properties of halogen atoms for representing intermolecular electrostatic interactions related to halogen bonding and their substituent effects. $J$. Comput. Chem. 2010, 31, 107-116.

(206) Jahromi, H.; Eskandari, K. Halogen bonding: a theoretical study based on atomic multipoles derived from quantum theory of atoms in molecules. Struct. Chem. 2013, $24,1281-1287$. 
(207) Torii, H. The role of atomic quadrupoles in intermolecular electrostatic interactions of polar and nonpolar molecules. J. Chem. Phys. 2003, 119, 2192-2198.

(208) Carter, M.; Rappé, A. K.; Ho, P. S. Scalable anisotropic shape and electrostatic models for biological bromine halogen bonds. J. Chem. Theory Comput. 2012, 8, 2461-2473.

(209) Scholfield, M. R.; Ford, M. C.; Vander Zanden, C. M.; Billman, M. M.; Ho, P. S.; Rappé, A. K. Force Field Model of Periodic Trends in Biomolecular Halogen Bonds. J. Phys. Chem. B 2015, 119, 9140-9149.

(210) Klebe, G. Virtual ligand screening: strategies, perspectives and limitations. Drug discovery today 2006, 11, 580-594.

(211) Schneider, G. Virtual screening: an endless staircase? Nat. Rev. Drug Discovery 2010, 9, $273-276$.

(212) Meng, E. C.; Shoichet, B. K.; Kuntz, I. D. Automated docking with grid-based energy evaluation. J. Comput. Chem. 1992, 13, 505-524.

(213) Morris, G. M.; Goodsell, D. S.; Halliday, R. S.; Huey, R.; Hart, W. E.; Belew, R. K.; Olson, A. J. Automated docking using a Lamarckian genetic algorithm and an empirical binding free energy function. J. Comput. Chem. 1998, 19, 1639-1662.

(214) Lang, P. T.; Brozell, S. R.; Mukherjee, S.; Pettersen, E. F.; Meng, E. C.; Thomas, V.; Rizzo, R. C.; Case, D. A.; James, T. L.; Kuntz, I. D. DOCK 6: Combining techniques to model RNA-small molecule complexes. RNA 2009, 15, 1219-1230.

(215) Stahl, M.; Rarey, M. Detailed analysis of scoring functions for virtual screening. J. Med. Chem. 2001, 44, 1035-1042.

(216) Schneider, G.; Böhm, H. Virtual screening and fast automated docking methods. Drug Discovery Today 2002, 7, 64-70. 
(217) Kuhn, B.; Fuchs, J. E.; Reutlinger, M.; Stahl, M.; Taylor, N. R. Rationalizing tight ligand binding through cooperative interaction networks. J. Chem. Inf. Model. 2011, $51,3180-3198$.

(218) Liu, Y.; Xu, Z.; Yang, Z.; Chen, K.; Zhu, W. A knowledge-based halogen bonding scoring function for predicting protein-ligand interactions. J. Mol. Model. 2013, 19, $5015-5030$.

(219) Muegge, I.; Martin, Y. C. A general and fast scoring function for protein-ligand interactions: a simplified potential approach. J. Med. Chem. 1999, 42, 791-804.

(220) Gohlke, H.; Hendlich, M.; Klebe, G. Knowledge-based scoring function to predict protein-ligand interactions. J. Mol. Biol. 2000, 295, 337-356.

(221) Zheng, M.; Xiong, B.; Luo, C.; Li, S.; Liu, X.; Shen, Q.; Li, J.; Zhu, W.; Luo, X.; Jiang, H. Knowledge-based scoring functions in drug design: 3 A two-dimensional knowledge-based hydrogen-bonding potential for the prediction of protein-ligand interactions. J. Chem. Inf. Model. 2011, 51, 2994-3004.

(222) Wang, R.; Fang, X.; Lu, Y.; Wang, S. The PDBbind database: collection of binding affinities for protein-ligand complexes with known three-dimensional structures. $J$. Med. Chem. 2004, 47, 2977-2980.

(223) Kolář, M.; Hobza, P.; Bronowska, A. K. Plugging the explicit $\sigma$-holes in molecular docking. Chem. Commun. 2013, 49, 981-983.

(224) Zimmermann, M. O.; Lange, A.; Boeckler, F. M. Evaluating the Potential of Halogen Bonding in Molecular Design: Automated Scaffold Decoration Using the New Scoring Function XBScore. J. Chem. Inf. Model. 2015, 55, 687-699.

(225) Wilcken, R.; Zimmermann, M. O.; Lange, A.; Zahn, S.; Boeckler, F. M. Using halogen 
bonds to address the protein backbone: a systematic evaluation. J. Comput.-Aided Mol. Des. 2012, 26, 935-945.

(226) Friesner, R. A.; Murphy, R. B.; Repasky, M. P.; Frye, L. L.; Greenwood, J. R.; Halgren, T. A.; Sanschagrin, P. C.; Mainz, D. T. Extra precision glide: docking and scoring incorporating a model of hydrophobic enclosure for protein-ligand complexes. J. Med. Chem. 2006, 49, 6177-6196.

(227) Jones, G.; Willett, P.; Glen, R. C. Molecular recognition of receptor sites using a genetic algorithm with a description of desolvation. J. Mol. Biol. 1995, 245, 43-53.

(228) Verdonk, M. L.; Cole, J. C.; Hartshorn, M. J.; Murray, C. W.; Taylor, R. D. Improved protein-ligand docking using GOLD. Proteins: Struct. Funct. 2003, 52, 609-623.

(229) Pecina, A.; Meier, R.; Fanfrlík, J.; Lepšík, M.; Řezáč, J.; Hobza, P.; Baldauf, C. Title. J. Chem. Inf. Model. 2016, 00, 0000-0000.

(230) Raha, K.; Merz, K. M. A quantum mechanics-based scoring function: study of zinc ion-mediated ligand binding. J. Am. Chem. Soc. 2004, 126, 1020-1021.

(231) Peters, M.; Raha, K.; Merz, K. M. Quantum mechanics in structure-based drug design. Curr. Opin. Drug Discovery Dev. 2006, 9, 370-379.

(232) Lommerse, J. P.; Stone, A. J.; Taylor, R.; Allen, F. H. The nature and geometry of intermolecular interactions between halogens and oxygen or nitrogen. J. Am. Chem. Soc. 1996, 118, 3108-3116.

(233) Bauzá, A.; Quiñonero, D.; Deyà, P. M.; Frontera, A. Halogen bonding versus chalcogen and pnicogen bonding: a combined Cambridge structural database and theoretical study. CrystEngComm 2013, 15, 3137-3144.

(234) Allen, F. H. The Cambridge Structural Database: a quarter of a million crystal structures and rising. Acta Crystallogr., Sect. B: Struct. Sci. 2002, 58, 380-388. 
(235) Auffinger, P.; Hays, F. A.; Westhof, E.; Ho, P. S. Halogen bonds in biological molecules. Proc. Natl. Acad. Sci. U. S. A 2004, 101, 16789-16794.

(236) Bernstein, F. C.; Koetzle, T. F.; Williams, G. J.; Meyer, E. F.; Brice, M. D.; Rodgers, J. R.; Kennard, O.; Shimanouchi, T.; Tasumi, M. The Protein Data Bank: a computer-based archival file for macromolecular structures. Arch. Biochem. Biophys. 1978, 185, 584-591.

(237) Tsuzuki, S.; Wakisaka, A.; Ono, T.; Sonoda, T. Magnitude and Origin of the Attraction and Directionality of the Halogen Bonds of the Complexes of C6F5X and C6H5X (X= I, Br, Cl and F) with Pyridine. Chem. Eur. J. 2012, 18, 951-960.

(238) Iwaoka, M.; Takemoto, S.; Tomoda, S. Statistical and Theoretical Investigations on the Directionality of Nonbonded SO Interactions Implications for Molecular Design and Protein Engineering. J. Am. Chem. Soc. 2002, 124, 10613-10620.

(239) Adhikari, U.; Scheiner, S. Sensitivity of pnicogen, chalcogen, halogen and H-bonds to angular distortions. Chem. Phys. Lett. 2012, 532, 31-35.

(240) Legon, A.; Millen, D. Directional character, strength, and nature of the hydrogen bond in gas-phase dimers. Acc. Chem. Res 1987, 20, 39-46.

(241) Bernstein, J.; Davis, R. E.; Shimoni, L.; Chang, N. Patterns in hydrogen bonding: functionality and graph set analysis in crystals. Angew. Chem., Int. Ed. 1995, 34, $1555-1573$.

(242) Wood, P. A.; Allen, F. H.; Pidcock, E. Hydrogen-bond directionality at the donor H atom-analysis of interaction energies and database statistics. CrystEngComm 2009, 11, 1563-1571.

(243) Platts, J.; Howard, S.; Bracke, B. Directionality of hydrogen bonds to sulfur and oxygen. J. Am. Chem. Soc. 1996, 118, 2726-2733. 
(244) Hobza, P.; Havlas, Z. Blue-shifting hydrogen bonds. Chem. Rev. 2000, 100, 42534264.

(245) Arunan, E.; Desiraju, G. R.; Klein, R. A.; Sadlej, J.; Scheiner, S.; Alkorta, I.; Clary, D. C.; Crabtree, R. H.; Dannenberg, J. J.; Hobza, P.; Kjaergaard, H. G.; Legon, A. C.; Mennucci, B.; Nesbitt, D. Definition of the hydrogen bond (IUPAC Recommendations 2011) Pure Appl. Chem. 2011, 83, 1637-1641.

(246) Politzer, P.; Murray, J. S.; Lane, P. $\sigma$-Hole bonding and hydrogen bonding: Competitive interactions. Int. J. Quantum Chem. 2007, 10\%, 3046-3052.

(247) Wang, W.; Zhang, Y.; Ji, B.; Tian, A. On the correlation between bond-length change and vibrational frequency shift in halogen-bonded complexes. J. Chem. Phys. 2011, $134,224303$.

(248) Murray, J. S.; Concha, M. C.; Lane, P.; Hobza, P.; Politzer, P. Blue shifts vs red shifts in $\sigma$-hole bonding. J. Mol. Model. 2008, 14, 699-704.

(249) Hermansson, K. Blue-shifting hydrogen bonds. J. Phys. Chem. A 2002, 106, 46954702.

(250) Qian, W.; Krimm, S. Vibrational Spectroscopy of Hydrogen Bonding: Origin of the Different Behavior of the CHO Hydrogen Bond. J. Phys. Chem. A 2002, 106, 66286636.

(251) Zierkiewicz, W.; Wieczorek, R.; Hobza, P.; Michalska, D. Halogen bonded complexes between volatile anaesthetics (chloroform, halothane, enflurane, isoflurane) and formaldehyde: a theoretical study. Phys. Chem. Chem. Phys. 2011, 13, 5105-5113.

(252) Ouvrard, C.; Le Questel, J.; Berthelot, M.; Laurence, C. Halogen-bond geometry: a crystallographic database investigation of dihalogen complexes. Acta Crystallogr., Sect. B: Struct. Sci. 2003, 59, 512-526. 
(253) Iwaoka, M.; Komatsu, H.; Katsuda, T.; Tomoda, S. Nature of Nonbonded Se...O Interactions Characterized by $17 \mathrm{O}$ NMR Spectroscopy and NBO and AIM Analyses. J. Am. Chem. Soc. 2004, 126, 5309-5317.

(254) Trnka, J.; Sedlak, R.; Kolář, M.; Hobza, P. Differences in the Sublimation Energy of Benzene and Hexahalogenbenzenes Are Caused by Dispersion Energy. J. Phys. Chem. A 2013, 117, 4331-4337.

(255) Sirimulla, S.; Bailey, J. B.; Vegesna, R.; Narayan, M. Halogen interactions in Protein-Ligand complexes: Implications of halogen bonding for rational drug design. $J$. Chem. Inf. Model. 2013, 53, 2781-2791.

(256) Cody, V.; Murray-Rust, P. Iodine...X (O, N, S) intermolecular contacts: models of thyroid hormone protein binding interactions using information from the cambridge crystallographic data files. J. Mol. Struct. 1984, 112, 189-199.

(257) Lu, Y.; Wang, Y.; Zhu, W. Nonbonding interactions of organic halogens in biological systems: implications for drug discovery and biomolecular design. Phys. Chem. Chem. Phys. 2010, 12, 4543-4551.

(258) Hartshorn, M. J.; Murray, C. W.; Cleasby, A.; Frederickson, M.; Tickle, I. J.; Jhoti, H. Fragment-based lead discovery using X-ray crystallography. J. Med. Chem. 2005, 48, 403-413.

(259) Battistutta, R.; Mazzorana, M.; Sarno, S.; Kazimierczuk, Z.; Zanotti, G.; Pinna, L. A. Inspecting the structure-activity relationship of protein kinase CK2 inhibitors derived from tetrabromo-benzimidazole. Chem. Biol. 2005, 12, 1211-1219.

(260) Estébanez-Perpiñá, E.; Arnold, L. A.; Nguyen, P.; Rodrigues, E. D.; Mar, E.; Bateman, R.; Pallai, P.; Shokat, K. M.; Baxter, J. D.; Guy, K. R.; Webb, P.; Fletterick, R. J. A surface on the androgen receptor that allosterically regulates coactivator binding. Proc. Natl. Acad. Sci. U. S. A 2007, 104, 16074-16079. 
(261) Riley, K. E.; Hobza, P. Strength and character of halogen bonds in protein-ligand complexes. Cryst. Growth Des. 2011, 11, 4272-4278.

(262) Darimont, B. D.; Wagner, R. L.; Apriletti, J. W.; Stallcup, M. R.; Kushner, P. J.; Baxter, J. D.; Fletterick, R. J.; Yamamoto, K. R. Structure and specificity of nuclear receptor-coactivator interactions. Genes Dev. 1998, 12, 3343-3356.

(263) Mueller-Dieckmann, C.; Ritter, H.; Haag, F.; Koch-Nolte, F.; Schulz, G. n. Structure of the ecto-ADP-ribosyl transferase ART2 2 from rat. J. Mol. Biol. 2002, 322, 687696.

(264) Battistutta, R.; Mazzorana, M.; Cendron, L.; Bortolato, A.; Sarno, S.; Kazimierczuk, Z.; Zanotti, G.; Moro, S.; Pinna, L. A. The ATP-Binding Site of Protein Kinase CK2 Holds a Positive Electrostatic Area and Conserved Water Molecules. Chembiochem 2007, 8, 1804-1809.

(265) Sarno, S.; Papinutto, E.; Franchin, C.; Bain, J.; Elliott, M.; Meggio, F.; Kazimierczuk, Z.; Orzeszko, A.; Zanotti, G.; Battistutta, R.; Pinna, L. ATP site-directed inhibitors of protein kinase CK2: an update. Curr. Top. Med. Chem. 2011, 11, 13401351.

(266) Fanfrlík, J.; Kolář, M.; Kamlar, M.; Hurný, D.; Ruiz, F. X.; Cousido-Siah, A.; Mitschler, A.; Řezáč, J.; Munusamy, E.; Lepšík, M.; Matějíček, P.; Veselý, J.; Podjarny, A.; Hobza, P. Modulation of aldose reductase inhibition by halogen bond tuning. ACS Chem. Biol. 2013, 8, 2484-2492.

(267) Fanfrlik, J.; Ruiz, F. X.; Kadlčíková, A.; Řezáč, J.; Cousido-Siah, A.; Mitschler, A.; Haldar, S.; Lepš́́k, M.; Kolář, M. H.; Majer, P.; Podjarny, A. D.; Hobza, P. The Effect of Halogen-to-Hydrogen Bond Substitution on Human Aldose Reductase Inhibition. ACS Chem. Biol. 2015, 10, 1637-1642. 
(268) Carter, M.; Ho, P. S. Assaying the energies of biological halogen bonds. Cryst. Growth Des. 2011, 11, 5087-5095.

(269) Voth, A. R.; Hays, F. A.; Ho, P. S. Directing macromolecular conformation through halogen bonds. Proc. Natl. Acad. Sci. U. S. A 2007, 104, 6188-6193.

(270) Carter, M.; Voth, A. R.; Scholfield, M. R.; Rummel, B.; Sowers, L. C.; Ho, P. S. Enthalpy-Entropy compensation in biomolecular halogen bonds measured in DNA junctions. Biochemistry 2013, 52, 4891-4903.

(271) Güssregen, S.; Matter, H.; Hessler, G.; Müller, M.; Schmidt, F.; Clark, T. 3D-QSAR based on quantum-chemical molecular fields: toward an improved description of halogen interactions. J. Chem. Inf. Model. 2012, 52, 2441-2453.

(272) Llinas-Brunet, M. et al. Discovery of a potent and selective noncovalent linear inhibitor of the hepatitis C virus NS3 protease (BI 201335). J. Med. Chem. 2010, 53, 6466-6476.

(273) Carpenter, R. D.; Natarajan, A.; Lau, E. Y.; Andrei, M.; Solano, D. M.; Lightstone, F. C.; De Nardo, S. J.; Lam, K. S.; Kurth, M. J. Halogenated Benzimidazole Carboxamides Target Integrin $\alpha 4 \beta 1$ on T-Cell and B-Cell Lymphomas. Cancer Res. 2010, 70, 5448-5456.

(274) Hardegger, L. A.; Kuhn, B.; Spinnler, B.; Anselm, L.; Ecabert, R.; Stihle, M.; Gsell, B.; Thoma, R.; Diez, J.; Benz, J.; Plancher, J.-m.; Hartmann, G.; Banner, D. W.; Haap, W.; Diederich, F. Systematic investigation of halogen bonding in Protein-Ligand interactions. Angew. Chem., Int. Ed. 2011, 50, 314-318.

(275) Hardegger, L. A. et al. Halogen bonding at the active sites of human cathepsin 1 and MEK1 kinase: Efficient interactions in different environments. ChemMedChem 2011, $6,2048-2054$. 
(276) Fedorov, O.; Huber, K.; Eisenreich, A.; Filippakopoulos, P.; King, O.; Bullock, A. N.; Szklarczyk, D.; Jensen, L. J.; Fabbro, D.; Trappe, J.; Rauch, U.; Bracher, F.; Knapp, S. Specific CLK inhibitors from a novel chemotype for regulation of alternative splicing. Chemistry \& Biology 2011, 18, 67-76.

(277) Himmel, D. M.; Das, K.; Clark, A. D.; Hughes, S. H.; Benjahad, A.; Oumouch, S.; Guillemont, J.; Coupa, S.; Poncelet, A.; Csoka, I.; Meyer, C.; Andries, K.; Nguyen, C. H.; Grierson, D. S.; Arnold, E. Crystal structures for HIV-1 reverse transcriptase in complexes with three pyridinone derivatives: a new class of Non-Nucleoside inhibitors effective against a broad range of Drug-Resistant strains. J. Med. Chem. 2005, 48, 7582-7591.

(278) Bollini, M.; Domaoal, R. A.; Thakur, V. V.; Gallardo-Macias, R.; Spasov, K. A.; Anderson, K. S.; Jorgensen, W. L. Computationally-Guided optimization of a docking hit to yield catechol diethers as potent Anti-HIV agents. J. Med. Chem. 2011, 54, 8582-8591.

(279) Wilcken, R.; Liu, X.; Zimmermann, M. O.; Rutherford, T. J.; Fersht, A. R.; Joerger, A. C.; Boeckler, F. M. Halogen-enriched fragment libraries as leads for drug rescue of mutant p53. J. Am. Chem. Soc. 2012, 134, 6810-6818.

(280) Chudzinski, M. G.; Taylor, M. S. Correlations between computation and experimental thermodynamics of halogen Bonding. J. Org. Chem. 2012, 77, 3483-3491. 
Graphical TOC Entry

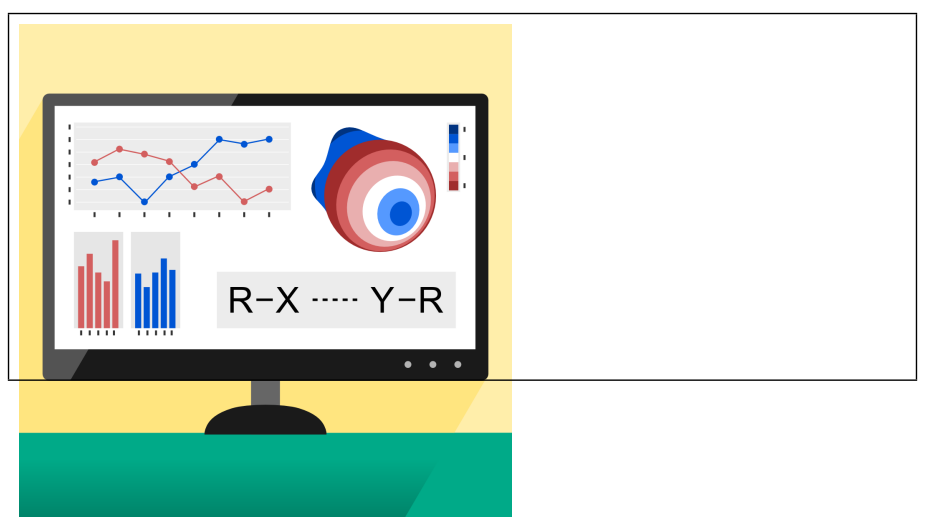

